\title{
A DIARY STUDY OF FEEDBACK SEEKING BEHAVIOURS IN INDIVIDUALS WITH SOCIAL ANXIETY DISORDER COMPARED TO INDIVIDUALS WITH GENERALIZED ANXIETY DISORDER OR NO HISTORY OF MENTAL HEALTH DIFFICULTIES
}

by

\author{
Gillian Wilson
}

Honours Bachelor of Arts, Queen's University, June 2012

\author{
A thesis presented to Ryerson University \\ in partial fulfillment of the \\ requirements for the degree of \\ Master of Arts \\ in the Program of \\ Psychology
}

Toronto, Ontario, Canada, 2015

(C) Gillian Wilson 2016 


\section{AUTHOR'S DECLARATION FOR ELECTRONIC SUBMISSION OF A THESIS}

I hereby declare that I am the sole author of this thesis. This is a true copy of the thesis, including any required final revisions, as accepted by my examiners.

I authorize Ryerson University to lend this thesis to other institutions or individuals for the purpose of scholarly research.

I further authorize Ryerson University to reproduce this thesis by photocopying or by other means, in total or in part, at the request of other institutions or individuals for the purpose of scholarly research.

I understand that my thesis may be made electronically available to the public. 
A Diary Study of Feedback Seeking Behaviours in Individuals With Social Anxiety Disorder Compared to Individuals With Generalized Anxiety Disorder or No History of Mental Health Difficulties

Master of Arts, 2016

Gillian Wilson

Psychology

Ryerson University

\begin{abstract}
The current study examined various features of positive feedback seeking (PFS) and negative feedback seeking (NFS) in individuals with social anxiety disorder (SAD), individuals with generalized anxiety disorder (GAD), and healthy individuals using a 2-week daily diary method. There were no significant differences between individuals with SAD and healthy individuals in the frequency of feedback seeking. However, individuals with GAD engaged in significantly more overall feedback seeking (adjusted for compliance) than healthy individuals. The most common source of feedback seeking within each group was other people (e.g., romantic partner). Individuals with SAD experienced significantly greater reductions in anxiety, sadness, and anger than healthy individuals and a significantly greater increase in certainty than individuals with GAD following positive feedback during PFS. There were no significant group differences in the topics, triggers, functions, or termination criteria of feedback seeking. Future research directions and theoretical and clinical implications of these findings are discussed.
\end{abstract}




\section{Acknowledgements}

I would like to thank my family, friends, and colleagues for their help and support throughout the process of completing my Master's thesis. I would also like to thank Dr. Martin Antony and Dr. Naomi Koerner for their helpful contributions and guidance in the development and completion of the current study. This study was conducted with the support of a Canadian Institutes of Health Research (CIHR) Frederick Banting and Charles Best Canada Graduate Scholarship. 


\section{Table of Contents}

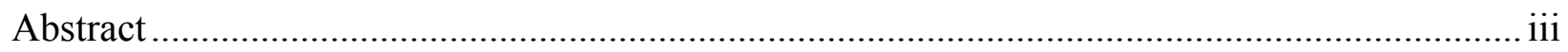

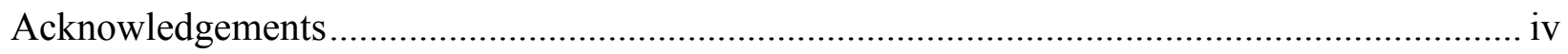

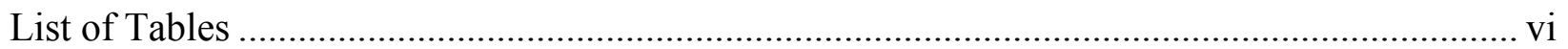

List of Figures .......................................................................................................... vii

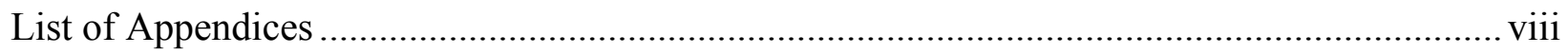

A Diary Study of Feedback Seeking Behaviours in Individuals With Social Anxiety Disorder

Compared to Individuals With Generalized Anxiety Disorder or No History of Mental Health

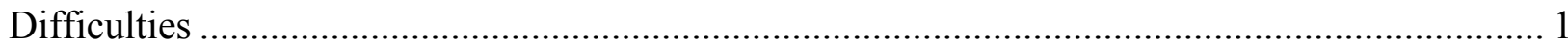

Excessive Reassurance Seeking and Social Anxiety .................................................. 3

Negative Feedback Seeking and Social Anxiety ............................................................. 7

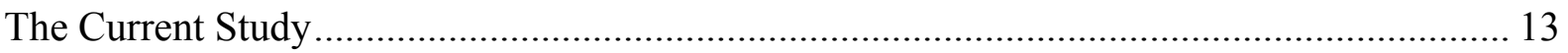

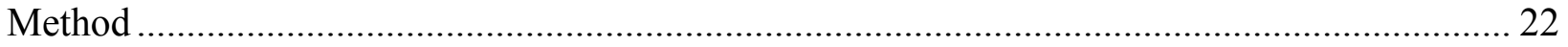

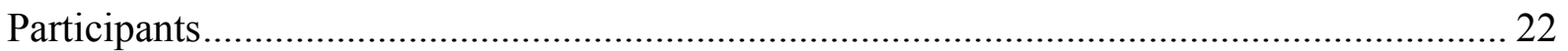

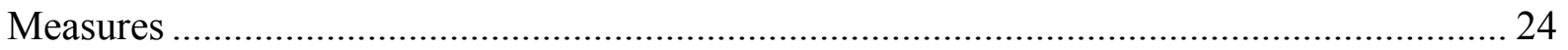

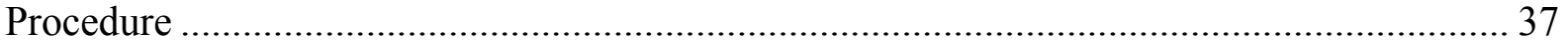

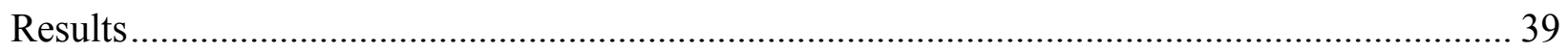

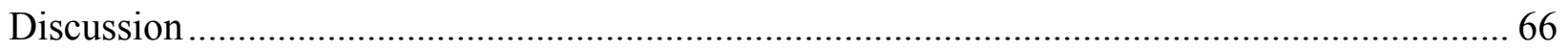




\section{List of Tables}

Table 1. Demographic Characteristics of the Sample Stratified by Group

Table 2. Descriptive Statistics for Frequency of Feedback Seeking Behaviours Over a 2-Week Period (Based on Diary Records) Stratified by Group

Table 3. Self-Report Questionnaire Scores Stratified by Group

Table 4. Regression Coefficients for Models Predicting Feedback Seeking Frequency from Group

Table 5. Correlations between Frequency of Feedback Seeking Behaviours Over a 2-Week Period (Based on Diary Records) and Scores on Feedback Seeking Questionnaires

Table 6. Mean Percentage of Topics of Overall Feedback Seeking Over a 2-Week Period (Based on Diary Records) Stratified by Group

Table 7. Mean Percentages of Sources of Overall Feedback Seeking Over a 2-Week Period (Based on Diary Records) Stratified by Group

Table 8. Mean Percentages of Triggers of Positive Feedback Seeking, Negative Feedback Seeking, and Overall Feedback Seeking Over a 2-Week Period (Based on Diary Records) Stratified by Group

Table 9. Mean Percentages of Functions of Positive Feedback Seeking and Negative Feedback Seeking Over a 2-Week Period (Based on Diary Records) Stratified by Group

Table 10. Mean Percentages of Termination Criteria of Positive Feedback Seeking and Negative Feedback Seeking Over a 2-Week Period (Based on Diary Records) Stratified by Group

Table 11. Mean Pre-, Post-, and Change Scores on Affective States, Certainty, and Uncertainty After Receiving Positive Feedback During Episodes of Positive Feedback Seeking

Table 12. Mean Pre-, Post-, and Change Scores on Affective States, Certainty, and Uncertainty After Receiving Negative Feedback During Episodes of Positive Feedback Seeking

Table 13. Mean Pre-, Post-, and Change Scores on Affective States, Certainty, and Uncertainty After Receiving Positive Feedback During Episodes of Negative Feedback Seeking

Table 14. Mean Pre-, Post-, and Change Scores on Affective States, Certainty, and Uncertainty After Receiving Negative Feedback During Episodes of Negative Feedback Seeking 


\section{List of Figures}

Figure 1. Regression lines of the predicted frequency of overall feedback seeking (adjusted for compliance ratings) from Intolerance of Uncertainty scores stratified by group (GAD group versus nonclinical comparison group).

Figure 2. Regression lines of the predicted frequency of positive feedback seeking from sources other than people (e.g., websites, books) from Intolerance of Uncertainty Scale scores stratified by group (SAD group versus nonclinical comparison group). 


\section{List of Appendices}

Appendix A: Telephone Screening Interview

Appendix B: Feedback Seeking Diary

Appendix C: Positive Feedback Seeking Frequency Questionnaire

Appendix D: Negative Feedback Seeking Frequency Questionnaire 
A Diary Study of Feedback Seeking Behaviours in Individuals With Social Anxiety Disorder

Compared to Individuals With Generalized Anxiety Disorder or No History of Mental Health

\section{Difficulties}

Social anxiety disorder (SAD) is one of the most common anxiety disorders, with a lifetime prevalence rate of $12.1 \%$ in the general population (Kessler, Berglund, Demler, Merikangas, \& Walters, 2005). The core feature of SAD is intense fear or anxiety circumscribed to at least one social situation in which there is a possibility of being negatively judged by others (American Psychiatric Association, 2013). Individuals with SAD experience intense fear or anxiety about behaving in a manner or exhibiting signs of anxiety (e.g., blushing, sweating, stuttering) that will be unfavourably appraised by others. The number and types of social situations that elicit marked fear or anxiety vary across individuals; whereas some individuals experience extreme fear or anxiety in very specific social situations (e.g., public speaking), others may experience these emotions every time they are in the presence of others (Antony \& Swinson, 2000). Commonly feared social situations include those involving potential observation by others (e.g., eating, drinking, or writing in the presence of others, using public restrooms, riding public transportation), performing in front of others (e.g., giving a formal presentation, speaking in class, playing sports), and social interactions (e.g., meeting new people, engaging in conversations, initiating and engaging in intimate or sexual encounters, interacting with authority figures). Social anxiety can be broken down into two broad categories that capture common social fears: public scrutiny or performance anxiety, which is experienced in situations in which an individual may be observed or overheard by others, but that do not involve direct social interaction, and social interaction anxiety, which refers to anxiety experienced in the context of social exchanges in dyads or groups (e.g., see Mattick \& Clarke, 1998; Weeks, 2015). 
To receive a diagnosis of SAD, individuals must either avoid feared social situations or experience a level of distress that is disproportionate to the present danger of the situation almost every time the situation is encountered (American Psychiatric Association, 2013). Consequently, SAD is associated with markedly impaired social functioning, as well as significant interference in occupational, academic, and physical domains (e.g., Antony, Roth, Swinson, Huta, \& Devins, 1998; Kessler, 2003), resulting in reduced quality of life compared to nonanxious individuals (Barrera \& Norton, 2009). Additionally, the course of SAD tends to be chronic and unremitting, particularly in individuals who fear a wide range of social situations (Keller, 2003), contributing to the negative long-term impact of this disorder on daily functioning and overall life satisfaction (Stein \& Kean, 2000).

Cognitive theories of SAD assume that a number of cognitive processes and behaviours play a role in the development and maintenance of this disorder (e.g., Clark \& Wells, 1995; Hofmann, 2007). According to cognitive models of SAD, socially anxious individuals experience fear and anxiety in response to high perceived social standards and doubts in their ability to meet these standards due to deficits in defining and achieving their social goals (e.g., making a positive social impression). The discrepancy between high perceived social expectations and an inability to fulfill these expectations leads to further anxiety and heightened self-focused attention, during which individuals shift their focus inward and engage in careful self-monitoring of internal thoughts, images, and physiological sensations, thereby failing to attend to important external information that could potentially disconfirm fears of negative evaluation (e.g., positive social cues from others) (Perowne \& Mansell, 2002). Heightened social apprehension and self-focused attention is believed to trigger a number of cognitive processes that further contribute to the maintenance of social anxiety, including overestimating the 
likelihood of a feared outcome occurring (e.g., behaving in a socially unacceptable manner) and its adverse consequences (e.g., humiliation, rejection), underestimating one's ability to control their anxiety, perceiving one's social skills as deficient, and experiencing negative self-images (e.g., images of the self as socially awkward or unattractive), which have been shown to have a detrimental impact on the quality of social interactions (e.g., Hirsch, Meynen, \& Clark, 2004). In an attempt to prevent the occurrence of feared outcomes and to lower distress, socially anxious individuals are proposed to engage in avoidance or safety behaviours, such as avoiding eye contact, gripping an object to mask shaking hands, or mentally rehearsing sentences before speaking (Clark \& Wells, 1995; Wells et al., 1995). As discussed in greater detail below, safety behaviours prevent disconfirmation of distorted beliefs about the probability of a negative outcome occurring and, in doing so, maintain social anxiety over time.

\section{Excessive Reassurance Seeking and Social Anxiety}

An examination of maladaptive behaviours in SAD can enhance our understanding of the profound functional impairment associated with this disorder. One such behaviour, which has been investigated extensively in major depressive disorder (MDD) (see Evraire \& Dozois, 2011; Starr \& Davila, 2008 for reviews) and in several anxiety-based problems, such as health anxiety and obsessive-compulsive disorder (OCD) (see Kobori \& Salkovskis, 2013 for a review), is excessive reassurance seeking (ERS), which has been referred to as positive feedback seeking (PFS) in the literature (e.g., Casbon, Burns, Bradbury, \& Joiner, 2005). In the context of anxiety, ERS has been defined as "the repeated solicitation of safety-related information from others about a threatening object, situation, or interpersonal characteristic despite having already received this information" (Parrish \& Radomsky, 2010, p. 211). Seeking reassurance or positive feedback is a common response under conditions of perceived threat or uncertainty (Kobori \& 
Salkovskis, 2013). Individuals may seek reassurance to determine whether their anxiety is out of proportion to the perceived threat or uncertainty in a given situation. Receiving sought-after reassurance from trusted others may reduce anxiety by assuring individuals that "everything will be alright" and providing affirmation that close others are available in times of distress, which may reinforce or increase the perceived security of one's interpersonal relationships, leading to reductions in negative affect (Evraire \& Dozois, 2011).

Although reassurance seeking may be an adaptive strategy in certain situations, previous research suggests that the tendency to seek reassurance excessively plays a crucial role in the maintenance and exacerbation of various anxiety-related problems (Helbig-Lang \& Petermann, 2010; Rector et al., 2011), including OCD (e.g., Kobori \& Salkovskis, 2013; Parrish \& Radomsky, 2006, 2010), health anxiety (e.g., Birnie et al., 2013; Salkovskis \& Warwick, 1986), generalized anxiety disorder (GAD) (e.g., Woody \& Rachman, 1994), and SAD (Cougle et al., 2012; Heerey \& Kring, 2007). ERS can been conceptualized as a safety behaviour (i.e., a behaviour designed to eliminate or reduce perceived threat and subsequent anxiety, but that instead maintains or worsens anxiety over time; Clark \& Gelder, 1996; Salkovskis, 1991). Safety behaviours are proposed to perpetuate anxiety when individuals learn to attribute the nonoccurrence of feared outcomes and subsequent, albeit temporary, reductions in anxiety to the safety behaviour itself rather than to the absence of actual threat. This is because individuals who engage in safety behaviours deny themselves the opportunity to learn that catastrophic outcomes are unlikely to occur even when safety behaviours are not performed (Antony \& Rowa, 2008). The tendency to misattribute the nonoccurrence of negative outcomes to safety behaviours prevents disconfirmation of distorted beliefs (e.g., the belief that a catastrophic outcome is likely to occur), which, in turn, hinders new learning (e.g., that a catastrophic outcome is, in fact, 
unlikely to occur) and thus perpetuates anxiety (see Clark, 1999). Further, safety behaviours may paradoxically intensify the very symptoms they are designed to reduce while eliciting negative reactions from others (Wells et al., 1995). For instance, a socially anxious individual may choose to sit alone at a social event to avoid saying or doing something that might be negatively evaluated by others, yet others may view this behaviour as socially inappropriate, thus validating the individual's initial fear of negative evaluation.

The same may be true of reassurance seeking, which has been conceptualized as an insituation "restorative" safety behaviour that is designed to decrease the perceived probability of feared outcomes (e.g., being negatively evaluated by others) or decrease the physiological symptoms of anxiety (e.g., heart palpitations) (Helbig-Lang \& Petermann, 2010). Socially anxious individuals may attribute the nonoccurrence of feared events and subsequent reductions in anxiety to the receipt of reassurance as opposed to the absence of real danger or to their ability to effectively cope with anxiety-provoking situations on their own (Helbig-Lang \& Petermann, 2010; Salkovskis, 1991). Thus, seeking reassuring or positive feedback from others may prevent exposure to evidence that disconfirms distorted beliefs, impede reduction in anxious arousal, and undermine personal coping skills, which may perpetuate fear and anxiety (Clark \& Wells, 1995). A recent study found that greater self-reported reassurance seeking that involved seeking confirmation from others that adverse outcomes would not occur (e.g., "Do you need reassurance from others that everything will be alright?") and that one was not being negatively evaluated by others (e.g., "Do you often find yourself asking others whether there is something wrong with you?") was associated with higher levels of social anxiety over and above the effects of depression, trait anxiety, and intolerance of uncertainty (IU) (Cougle et al., 2012). Further, reassurance seeking concerning the occurrence of negative outcomes predicted increases in 
social anxiety 1 month later, whereas changes in social anxiety did not prospectively predict changes in reassurance seeking, suggesting a potential causal role of reassurance seeking in social anxiety, which is consistent with cognitive-behavioural models that implicate reassurance seeking as a type of safety behaviour that contributes to the maintenance and worsening of anxiety (see Antony \& Rowa, 2008; Clark \& Wells, 1995). However, Cougle and colleagues used a nonclinical, analogue student sample with only $19 \%$ of participants scoring within the clinical range on the Liebowitz Social Anxiety Scale Self Report Version (Cox, Ross, Swinson, \& Derenfeld, 1998), a self-report measure of social anxiety and avoidance, thereby raising questions about the generalizability of these findings to clinical samples in which diagnoses are determined by way of a structured or semistructured diagnostic interview.

Another study found that during a 5-minute interaction in which participants were instructed to "get to know one another," socially anxious individuals engaged in significantly more reassurance seeking than did nonanxious individuals (Heerey \& Kring, 2007). Although the interaction partners of socially anxious individuals provided more empathy and support during the conversation compared to the interaction partners of nonsocially anxious individuals, socially anxious individuals' bids for reassurance predicted reduced partner-rated interaction quality. The interaction partners of socially anxious individuals rated their interactions as significantly less rewarding than individuals who interacted with nonsocially anxious individuals, highlighting the negative interpersonal consequences of reassurance seeking in the context of social anxiety. The authors of this study proposed that reassurance seeking is a type of safety behaviour designed to reduce anxiety that stems, at least in part, from socially anxious individuals' tendency to engage in self-focused attention during social interactions, and is therefore used as an emotion regulation strategy that functions similarly to compulsive checking behaviour. Thus, reassurance seeking 
may be negatively reinforced in the short-term by reducing negative affect, but in the long-term this behaviour may have negative interpersonal consequences. Taken together, these findings suggest that although in-situation requests for reassurance may elicit empathy and support from others, reassurance seeking is perceived as a socially aversive behaviour, which may reduce others' desire to engage in future social interactions with an individual who frequently solicits reassurance. A decrease in social interactions may, in turn, validate negative self-beliefs surrounding social skills and social competence and thus perpetuate symptoms of social anxiety. Similar to the previously described study by Cougle and colleagues (2012), the study by Heerey and Kring (2007) included a student analogue sample, thereby raising questions about the generalizability of these findings to clinical samples.

Negative Feedback Seeking and Social Anxiety

There is evidence that socially anxious individuals engage in another form of maladaptive feedback seeking called negative feedback seeking (NFS). NFS is considered a self-verification process whereby individuals with negative self-beliefs desire and therefore preferentially seek negative self-referent information (Casbon et al., 2005; see Joiner, 2000). Although the desire to receive negative self-verifying information seems counterintuitive, self-verification theory (Swann, 1983) argues that individuals inherently value predictability and stability in how they view themselves (Swann, Stein-Seroussi, \& Giesler, 1992). Individuals may attempt to maintain stable self-images by preferentially interacting with others who perceive the individual as he or she perceives him- or herself (Swann et al., 1992) and by verbally requesting self-relevant feedback from others with the hope of receiving information that confirms and reinforces selfbeliefs (Swann \& Read, 1981a, 1981b). That is, individuals may "behave in ways that tend to bring their social environments into harmony with these images" (Swann \& Read, 1981a. pp. 
1127) because they are motivated to preserve preexisting self-views (Casbon et al., 2005). For example, a socially anxious individual may ask her partner whether she appeared uncomfortable at a social gathering with the hope of receiving feedback that confirms long-standing self-beliefs concerning perceived social deficits.

According to the self-verification model, self-confirmatory information is cognitively satisfying since it validates self-beliefs (Casbon, Burns, Bradbury, \& Joiner, 2005; Swann \& Reader, 1981a), even when these beliefs are negative (Joiner, 2000). Self-incongruent feedback, however, may be disregarded or perceived as threatening since it challenges well-established self-beliefs (Swann et al., 1992). In addition, if others fail to view an individual as he or she views him- or herself, the individual may fear that future interactions will lack a certain level of predictability and understanding. For example, if a socially anxious individual's partner appraises her as highly competent in social situations, she may have fears about meeting those expectations and the consequences of failing to do so (e.g., disappointing her partner).

There is a considerable amount of empirical support for the self-verification model (e.g., Casbon et al., 2005; Joiner, 2000; Swann \& Reader, 1981a, 1982b; Swann, Stein-Seroissi, \& Giesler, 1992). Swann and Read (1981a) found that individuals who perceived themselves as assertive preferentially sought feedback that confirmed their assertiveness whereas self-identified unassertive individuals preferentially requested feedback that verified their unassertiveness. The same pattern was observed for emotional versus unemotional individuals. Individuals reported a desire to receive self-verifying feedback because they considered this information to be more diagnostic and informative than feedback that disconfirmed self-views (Swann \& Read, 1981a).

Also consistent with self-verification theory is research demonstrating that individuals who viewed themselves as having low sociability preferentially selected interaction partners who 
appraised them unfavourably (Swann, Stein-Seroussi, \& Giesler, 1992). In this study, participants were provided with two fabricated evaluations of their social competence that were supposedly based on previously administered questionnaires and written by potential interaction partners with whom participants were told they would later have the opportunity to interact. One written evaluation was positive (e.g., "this person seems socially self-confident") whereas the other evaluation was negative (e.g., "he appears to be ill at ease in social situations"). Participants were asked to verbalize their thoughts out loud as they selected one of the two potential interaction partners based on the written evaluations. The purpose of having participants spontaneously verbalize their thoughts was to uncover their reasons for selecting interaction partners whose evaluations were self-verifying. The most common reason why individuals with low self-perceived sociability selected negative evaluators as interaction partners was because receiving negative appraisals confirmed their self-concepts, which was considered an "epistemic" reason for self-verification. The following verbalization exemplifies an epistemic concern: "I think the negative evaluator is the better choice because he sees me for who I really am." Individuals also selected interaction partners because of pragmatic and perceptiveness considerations, albeit to a lesser degree. Individuals' pragmatic concerns involved wanting to ensure that their partners viewed them as they viewed themselves and that their partners had realistic expectations of them to facilitate smooth interactions, whereas perceptiveness concerns involved selecting a partner based on their perceived level of insight and intelligence.

Similar self-verification processes have been demonstrated among married individuals (Swann, De La Ronde, \& Hixon, 1994). Swann and colleagues (1994) found that individuals with positive self-views showed a preference for spouses who appraised them positively, 
whereas individuals with negative self-views favoured spouses who appraised them negatively. Married individuals with negative self-views also demonstrated a unique tendency to reduce intimacy toward their spouse if they perceived that their spouse was overvaluing them, presumably because they wanted their spouses' evaluations to remain consistent with their selfbeliefs to sustain a sense of control and predictability in their marriage (e.g., to ensure that their spouses had realistic expectations of them).

Based on these findings, it is possible that individuals with SAD behave in a way that will elicit negative feedback from others because this type of feedback is consistent with their self-views (Clark \& Wells, 1995; Turner et al., 2003). Previous research has demonstrated that socially anxious individuals experience involuntary, recurrent, negative self-images in threatening social situations, which they erroneously perceive as accurate portrayals of how they behave and appear to others (Hackmann, Surway, \& Clark, 1998). The content of these negative self-images represent feared negative outcomes of socially anxious individuals, such as blushing or sweating excessively, rather than how others actually perceive them in that moment (Hirsch, Meynen, \& Clark, 2004). Hirsch and colleagues (2004) demonstrated that eliciting negative selfimages in socially anxious individuals (using an experimental manipulation) led to increased anxiety and greater use of safety behaviours (as reported by socially anxious participants), as well as more signs of anxiety, poorer overall performance (e.g., increased awkwardness), and lower quality social interactions (as reported by both the socially anxious individuals and their nonanxious interaction partners). Proponents of self-verification theory would propose that socially anxious individuals with negative self-images engage in particular behaviours in order "to bring their social environments into harmony with these images" (Swann \& Read, 1981a. pp. 1127) perhaps because they are motivated to preserve long-standing, negative self-views. 
Further, recent research found a significant positive association between social anxiety and a preference for negative self-relevant feedback to positive feedback (Valentiner, Skowronski, McGrath, Smith, \& Renner, 2011), which may serve to validate negative selfimages and beliefs (e.g., "I am socially incompetent") and, in turn, maintain social anxiety (North \& Swann, 2009). In the Valentiner and colleagues (2011) study, preference for negative feedback was measured using a modified version of the Feedback Seeking Questionnaire (FSQ: Swann et al., 1992a), which requires participants to select two questions from a number of selfrelevant domains (e.g., social, intellectual, physical appearance) that they would want someone close to them to answer about them. The questions are framed in positive terms (e.g., "What do you see as [participant's name] most physically attractive features?") or negative terms (e.g., “What do you see as [participant's name] least physically attractive features?"), and the number of negatively framed questions that participants select is used as an index of participants' preference for negative feedback over positive feedback.

Although self-verifying information may be cognitively satisfying, insomuch that it provides a sense of psychological coherence, it seems to have deleterious emotional consequences for individuals with negative self-beliefs, including reductions in self-esteem and the maintenance or worsening of negative affect (see North \& Swann, 2009). In other words, negative self-verifying information is cognitively satisfying for individuals with negative selfimages, but emotionally unpleasant, a phenomenon that has been termed the "cognitive-affective crossfire" (Swann, Griffin, Predmore, \& Gaines, 1987). The "cognitive-affective crossfire," which has been discussed extensively in the context of depression (e.g., Casbon et al., 2005; Joiner, 2000; Joiner, Alfano, \& Metalsky, 1993; Joiner \& Metalsky, 1995), may explain why socially anxious individuals engage in both ERS (or PFS) and NFS. Similar to depressed 
individuals, socially anxious individuals may seek reassurance or positive feedback about themselves to alleviate negative affect. Although emotionally satisfying, positive feedback may be perceived as cognitively incongruent with negative self-beliefs, leading individuals to surround themselves with people who view them unfavourably and to actively solicit negative self-confirmatory feedback, which is cognitively satisfying, but emotionally aversive. This may generate further ERS, and the cycle continues (Joiner et al., 1993). Lastly, there is some evidence that the tendency to alternate between ERS and NFS (depending on an individual's present desires to self-enhance versus self-verify) in the context of negative affect can lead to negative evaluation and rejection by others, which may further perpetuate poor self-esteem and low mood (Joiner \& Metalsky, 1995).

Furthermore, there is recent evidence that socially anxious individuals fear both negative and positive evaluation from others (Weeks, Heimberg, \& Rodebaugh, 2008; Weeks \& Howell, 2012), which may, in part, underlie feedback seeking behaviours in SAD. As previously delineated, concerns of being unfavourably appraised by others, which is a core feature of SAD, may motivate socially anxious individuals to seek reassurance to assure themselves that they did not behave in a manner than could elicit negative evaluation from others. Although the receipt of positive feedback may alleviate immediate distress, research has demonstrated an association between social anxiety and fear of positive evaluation (Weeks \& Howell, 2012). According to the bivalent fear of evaluation (BFOE) model, socially anxious individuals are apprehensive about receiving positive feedback and appearing "too good" due to concerns of posing a threat to individuals of a higher social ranking, which could potentially lead to social conflict (Weeks \& Howell, 2012). Weeks and Howell (2012) provided preliminary support for this model, demonstrating a significant relation between fear of positive evaluation and concerns of social 
reprisal as a result of making favourable impressions on others. An alternative explanation for why socially anxious individuals fear positive evaluation may be concerns or doubts about meeting others' high expectations for them in future interactions (Gilbert, 2001). Indeed, previous research has demonstrated that following positive social experiences, socially anxious individuals feared that their interaction partners would set unrealistically high standards for them in subsequent interactions that they would fail to meet (Wallace \& Alden, 1995).

Taken together, socially anxious individuals may fear and therefore dismiss or disqualify positive feedback for the following reasons: preservation of negative self-views, fear of social competition and retaliation, or concerns about failing to meet others' high expectations in future social interactions. Although merely a speculation (by the author), it is possible that following positive social experiences, including the receipt of positive feedback, socially anxious individuals attempt to undermine or dismiss these experiences by subsequently seeking negative feedback as a means to reinforce negative self-beliefs, reestablish their inferior status in order to reduce the risk of social reprisal, and lower others' expectations of them.

\section{The Current Study}

There is a paucity of research examining feedback seeking behaviours in anxiety-based problems, particularly in the context of SAD. The limited research that does exist demonstrates that these behaviours can have negative intra- and interpersonal consequences for socially anxious individuals. As previously discussed, reassurance seeking was found to prospectively predict increases in social anxiety (Cougle et al., 2012) and reduce partner-rated interaction quality (Heerey \& Kring, 2007), whereas the desire to solicit negative feedback, which may stem from epistemic concerns about preserving long-standing negative self-beliefs (Swann et al., 1992), and the actual receipt of negative feedback may reinforce negative self-images of socially 
anxious individuals (Swann et al., 1992; Valentiner et al., 2011). Therefore, although these two forms of feedback seeking may serve different functions-ERS (or PFS) may be best conceptualized as a safety behaviour designed to temporarily reduce anxiety and NFS as a behaviour designed to achieve psychological coherence, preserve one's perceived social status, or prevent increases in others' expectations — both may play an important role in the maintenance of social anxiety. As described earlier, cognitive models of SAD emphasize the role of negative self-images, which may motivate NFS, and safety behaviours (e.g., avoiding eye contact, gripping objects) in the maintenance of social anxiety (Clark \& Wells, 1995; Hofmann, 2007); however, feedback seeking behaviours are rarely discussed within these models (Wells et al., 1995), perhaps due to limited research investigating these behaviours in SAD. The current study aimed to address this gap in the literature by examining how feedback seeking behaviours naturally manifest in the context of SAD, which could have implications for advancing the cognitive model of SAD by incorporating feedback seeking as another safety behaviour that contributes to the maintenance of social anxiety. To gain a better understanding of how PFS and NFS manifest in SAD, the current study aimed to elucidate various features of feedback seeking behaviours, including the frequency, triggers, topics, sources, functions, termination factors, and affective consequences of these behaviours in individuals with SAD. Importantly, in the current study, the term PFS was used instead of ERS in order to clearly differentiate this form of feedback seeking from NFS. PFS was broadly defined as seeking and wanting positive, reassuring self-relevant information from other individuals (e.g., romantic partner, friend), oneself, or external sources (e.g. websites, books), whereas NFS was defined as seeking and wanting negative self-relevant information from these different sources. 
Individuals with GAD and individuals with no lifetime history of mental health difficulties were included as comparison groups to determine whether certain forms and features of feedback seeking, which may be a transdiagnostic behavioural manifestation of anxiety across various anxiety disorders (Cougle et al., 2012), are specific to social anxiety. Individuals with GAD were chosen as the clinical comparison group in light of previous research demonstrating a significant association between elevated levels of self-reported ERS and greater symptoms of GAD after controlling for depressive symptoms, trait anxiety, and IU, suggesting that the association between ERS and GAD is not better accounted for by related psychological constructs (Cougle et al., 2012). Similar to the association between reassurance seeking and SAD (described earlier), reassurance seeking predicted increases in GAD symptoms 1 month later, whereas GAD symptoms did not prospectively predict changes in reassurance seeking, indicating a directional effect in which ERS contributes to GAD symptoms and not vice versa. Further, Beesdo-Baum and colleagues (2012) found that individuals with GAD reported engaging in significantly more reassurance seeking than healthy individuals as an attempt to control or reduce worry, and that behavioural therapy (worry exposure and applied relaxation) was associated with significant reductions in reassurance seeking in individuals with GAD. To the author's knowledge, no research to date has examined potential associations between GAD and NFS. However, GAD is associated with dysfunctional beliefs surrounding one's ability to cope with uncertainty and solve problems (Robichaud \& Dugas, 2005). Self-verification theory would therefore posit that individuals with GAD may prefer and seek negative feedback about their problem-solving abilities in order to verify these negative self-beliefs.

The core feature of GAD is excessive and difficult-to-control worry about various topics and activities (e.g., concerns about safety, health, financial status, decision-making; American 
Psychiatric Association, 2013), which may lead these individuals to seek feedback in various contexts and from a variety of sources, such as family members, friends, authority figures, and healthcare professionals, perhaps as an attempt to alleviate uncertainty and achieve a sense of security in a number of life domains (see Woody \& Rachman, 1994). The primary concerns of individuals with SAD, on the other hand, are believed to be less diffuse, focusing more on social and evaluative concerns (e.g., fear of behaving in a manner or exhibiting signs of anxiety that may lead to negative evaluation by others), which may underlie more narrow forms of feedback seeking behaviours compared to those observed in individuals with GAD. However, social concerns are also common in individuals with GAD, raising the question of whether feedback seeking focusing on social/evaluative concerns is unique to individuals with SAD or a shared behaviour across these two anxiety disorders. Further, the rationale for including a nonclinical comparison group was to evaluate whether feedback seeking behaviours manifest differently in individuals with SAD versus healthy individuals, which (if this were the case) would suggest that these behaviours may contribute to social anxiety and should therefore be targeted in the treatment of SAD. If no significant differences arise in how these behaviours manifest, it may be that feedback seeking in SAD is a normative behaviour (such that it manifests similarly in healthy individuals) and therefore does not warrant significant clinical attention.

A naturalistic diary method was adapted from previous research and implemented for the current study (Antony, Rowa, Liss, Swallow, \& Swinson, 2005; Wheeler \& Miyake, 1992). Participants were required to complete a feedback-seeking diary record each time that they engaged in either PFS or NFS over a 2-week period. The number of completed diary records provided an estimate of feedback seeking frequency. For each diary entry, participants were instructed to record the type of feedback seeking in which they engaged (PFS or NFS); what 
triggered their feedback seeking; the topics/domains on which their feedback seeking centered (e.g., life achievements, signs of anxiety, personal safety); the sources of feedback (e.g., romantic partner, family member, professional/authority figure); reasons for seeking feedback (i.e., functions of feedback seeking); and termination criteria for a given feedback seeking episode. Further, the vast majority of research on PFS and NFS has been conducted in the area of depression, which has consistently demonstrated a significant, positive association between depressive symptoms and levels of feedback seeking (see Evraire \& Dozois, 2011). In addition, cognitive models of GAD propose that reassurance seeking is a behavioural manifestation of IU (Dugas \& Robichaud, 2007), which is defined as the tendency to respond negatively to uncertainty and its perceived consequences (Dugas, Gagnon, Ladouceur, \& Freeston, 1998b; Sexton \& Dugas, 2009). IU has also been shown to explain a significant amount of variance in social anxiety (Boelen \& Reijntjes, 2009), leading researchers to speculate that socially anxious individuals may engage in safety behaviours, such as feedback seeking, to alleviate feelings of uncertainty in social situations. As discussed earlier, there is evidence that socially anxious individuals fear both negative and positive evaluation (Weeks et al., 2008; Weeks \& Howell, 2012), which may also motivate feedback seeking behaviours in socially anxious individuals. Consequently, depressive symptoms, IU, fear of negative evaluation (FNE), and fear of positive evaluation (FPE) were examined as potential moderators to determine whether these constructs influenced significant associations between diagnostic group and various features of feedback seeking (e.g., whether individuals with SAD engaged in more PFS than healthy individuals, but only at high levels of depression). Trait anxiety, which is defined as a dispositional tendency to experience anxiety most of the time (Spielberger, 1983), was also examined as a moderator to determine whether diagnostic group was associated with features of feedback seeking 
irrespective of a general tendency to experience stable levels of anxiety. The aim of using a diary method was to examine how feedback seeking behaviours manifest on a daily basis in naturally occurring contexts, which has a number of advantages over the self-report and observational methods that have been used in previous studies investigating feedback seeking behaviours in the context of SAD (e.g., Cougle et al., 2012; Heerey \& Kring, 2012), including increased ecological validity and reduced recall bias (Gunthert \& Wenze, 2012).

Based on the reviewed literature, the following questions and hypotheses were advanced:

Hypothesis 1: Individuals with SAD and GAD were predicted to engage in significantly more PFS and NFS than individuals in the nonclinical comparison group (i.e., individuals with no lifetime history of mental health difficulties).

Hypothesis 2: Given the cognitive and behavioural features of SAD (Clark \& Wells, 1995), individuals with SAD were expected to engage in feedback seeking that is particularly associated with perceived social and evaluative threats (e.g., social competence, physical appearance). Specifically, it was predicted that the topics of feedback seeking behaviours in individuals with SAD would focus primarily on social/evaluative threats as compared to more general threats (e.g., health, safety, decision-making). Further, given the cognitive and behavioural features of GAD (Woody \& Rachman, 1994), individuals with GAD were expected to engage in feedback seeking behaviours that focused on a wider range of threats, including both social/evaluative threats and more general threats (e.g., health, safety, decision-making). Accordingly, it was predicted that there would be no differences between individuals with SAD and individuals with GAD in their tendencies to seek feedback focused on social/evaluative concerns, whereas the tendency to seek feedback focused on general safety/security concerns would be significantly greater in individuals with GAD than in individuals with SAD. 
Hypothesis 3: Given the wide variety of worries reported by people with GAD (Woody \& Rachman, 1994), individuals with GAD were predicted to seek feedback from a significantly greater number of sources than both individuals with SAD and nonclinical individuals, whereas individuals with $\mathrm{SAD}$ were predicted to seek feedback from a significantly greater number of sources than nonclinical individuals.

Hypothesis 4: Given the cognitive and behavioural features of SAD (Clark \& Wells, 1995) and GAD (Woody \& Rachman, 1994), it was predicted that there would be no differences between individuals with SAD and individuals with GAD in their tendencies to seek feedback triggered by social/evaluative concerns, whereas the tendency to seek feedback triggered by general safety/security concerns would be significantly greater in individuals with GAD compared to individuals with SAD.

Hypothesis 5: Given the cognitive features of individuals with SAD and individuals with GAD, it was predicted that reductions in social/evaluative threats would be a central function of PFS in individuals with SAD whereas reductions in both social/evaluative and general safety/security threats would be a primary function of PFS in individuals with GAD. More specifically, it was predicted that there would be no significant differences between these two groups in PFS aimed at reducing social/evaluative concerns, whereas individuals with GAD would engage in significantly more PFS aimed at reducing general threats.

Hypothesis 6: Consistent with self-verification theory and previous research (delineated above), it was predicted that one of the most common functions of NFS in both clinical groups would be the confirmation of negative self-views. Further, building on previous findings (described earlier), it was predicted that individuals with SAD would be more likely than individuals with GAD and nonclinical individuals to seek negative feedback in order to avoid 
threatening the social hierarchy by appearing "too good" in front of others, and to lower the expectations that others hold of them. In other words, it was predicted that there would be no significant differences between individuals with SAD and individuals with GAD in the tendency to engage in NFS designed to confirm negative self-views, whereas individuals with SAD would engage in significantly more NFS designed to avoid appearing "too good" and to lower others" expectations of them compared to the GAD group and the nonclinical comparison group.

Hypothesis 7: Given the cognitive features of individuals with SAD and individuals with GAD, it was predicted that there would be no significant differences between these two groups in the tendency to terminate PFS following reductions in social/evaluative concerns, whereas individuals with GAD would be significantly more likely to terminate PFS following reductions in general safety/security threats.

Hypothesis 8: It was predicted that one of the most common termination criteria of NFS in both clinical groups would be the confirmation of negative self-views. Further, it was predicted that individuals with SAD would be more likely than individuals with GAD and healthy individuals to terminate NFS once the threat of appearing "too good" in front of others was reduced or once they perceived that others' expectations of them were lowered. In other words, there would be no significant differences between the two clinical groups in the tendency to terminate NFS due to confirming negative self-views, whereas individuals with SAD would be significantly more likely than the other two groups to terminate NFS because the threat of appearing "too good" was decreased or others' expectations were lowered.

Hypothesis 9: First, it was predicted that the receipt of positive feedback would lead to decreases in negative affective states (e.g., anxiety, anger) and increases in positive affective states (e.g., happiness, relaxation) in individuals with GAD and healthy individuals, whereas the 
receipt of positive feedback would lead to increases in negative affective states and decreases in positive affective states in individuals with $\mathrm{SAD}$, which is consistent with the BFOE model (Weeks \& Howell, 2012). Second, it was predicted that the receipt of negative feedback would lead to increases in negative affective states and decreases in positive affective states within each group. Third, it was predicted that the receipt of either type of feedback (positive or negative) would lead to decreases in uncertainty and increases in certainty, particularly when the type of feedback received was congruent with the type of feedback hoped for (i.e., receiving positive feedback during episodes of PFS and receiving negative feedback during episodes of NFS).

Exploratory Question: Would there be significant group differences in how much negative affective states, positive affective states, uncertainty, and certainty changed following the receipt of positive and negative feedback during episodes of PFS and NFS? 


\section{Method}

\section{Participants}

The current study included 70 participants belonging to one of three groups: 1) SAD group ( $n=24), 2)$ GAD group ( $n=23)$, and 3) nonclinical comparison group (or healthy group) $(n=23)$. To be eligible for the SAD group, individuals had to have a principal diagnosis of SAD and no diagnosis of GAD. To be eligible for the GAD group, individuals had to have a principal diagnosis of GAD and no diagnosis of SAD. Individuals for whom diagnostic criteria were met for both SAD and GAD were not eligible to participate. Individuals in the SAD or GAD groups could have other comorbid diagnoses as long as SAD or GAD were the principal diagnoses, respectively, as determined by the Mini International Neuropsychiatric Interview for DSM-5 Disorders (MINI-5; Sheehan, 2014), a brief structured diagnostic interview that participants completed over the telephone (see Procedure). To be eligible for the nonclinical comparison group, individuals had to have no lifetime history of mental health difficulties. Consistent with previous research (Parrish \& Radomsky, 2010), participants who denied engaging in either ERS or NFS in the past 6 months were excluded. Participants were recruited through paper advertisements (Appendix A) posted in various public locations in the downtown Toronto area (e.g., university campuses, grocery stores, coffee shops, and bus shelters), as well as online advertisements posted on Kijiji, Craigslist, Facebook, and Reddit. All potential participants completed the MINI-5 as well as additional questions (Appendix B) based on DSM-5 diagnostic criteria to assess for anxiety-related disorders (e.g., specific phobia, body dysmorphic disorder) that are not included in the MINI to determine study eligibility. All participants received $\$ 30$ incentive following study completion. 
A total of 151 potential participants completed the telephone interview. Of these individuals, $31(20.53 \%)$ were deemed not eligible for having neither a current principal diagnosis of SAD nor GAD (e.g., met criteria for a different principal diagnosis, such as MDD, agoraphobia, or a personality disorder, or had subthreshold SAD or GAD) and $30(19.87 \%)$ were deemed not eligible for meeting diagnostic criteria for both SAD and GAD. Additional exclusion criteria for the clinical groups included a diagnosis of substance use disorder in the past 3 months for which five (3.31\%) individuals were excluded, evidence of psychotic disorder in the past 6 months for which two $(1.32 \%)$ individuals were excluded, and evidence of uncontrolled bipolar disorder (i.e., a manic or hypomanic episode in the past 6 months) for which four $(2.65 \%)$ individuals were excluded. Consistent with previous research (Parrish \& Radomsky, 2010), one $(0.66 \%)$ individual with GAD was excluded for reporting an absence of feedback seeking behaviours (ERS or NFS) in the past 6 months. All participants had to be at least 17 years of age, report a good level of proficiency in the English language, and have daily Internet access. Two individuals $(1.32 \%)$ were excluded due to poor or fair proficiency in the English language. Following completion of the telephone interview, four (2.65\%) eligible individuals were no longer interested in taking part in the study and two $(1.32 \%)$ individuals did not show up to their first laboratory visit. Two (1.32\%) participants were excluded from data analyses as they demonstrated limited understanding of the diary recording protocol, including the definitions of feedback seeking behaviours. Further, two (1.32\%) participants were excluded from data analyses because more than $20 \%$ of their diary records were removed due to missing data, and five $(3.31 \%)$ participants were excluded for being outliers on self-report questionnaires or diary records. Thus, a total of 90 telephone-screened individuals were ineligible (based on the telephone interview) or excluded from analyses for the reasons listed above, resulting in a final 
sample size of 61 (44 females, 16 males), ranging in age from 17 to 69 years $(M=26.92$ years, $S D=11.56)$, with 21 participants in the SAD group, 20 in the GAD group, and 20 in the nonclinical comparison group. The demographic characteristics of participants stratified by group are presented in Table 1.

\section{Measures}

Mini International Neuropsychiatric Interview for DSM-5 (MINI-5; Sheehan, 2014). The MINI is a brief, structured diagnostic interview that derives its diagnoses from criteria outlined in the DSM-5 to determine the presence of major forms of psychopathology as well as suicidality. The full MINI was conducted over the telephone with all potential participants to determine study eligibility. The author of the current study, a Master's student with extensive training in conducting diagnostic interviews, administered all telephone interviews. Previous versions of the MINI have demonstrated strong psychometric properties, including good convergent validity with other widely used diagnostic interviews (e.g., the Structured Clinical Interview for DSM-III-R Disorders, Patient Edition (SCID-P; Spitzer, Williams, Gibbon, \& First, 1999), excellent interrater reliability, very good test-retest reliability, and $85 \%$ diagnostic agreement between general practitioners and expert psychiatrists, with the highest level of agreement for diagnoses of MDD, GAD, and SAD (Sheehan et al., 1998).

Social Phobia Inventory (SPIN; Connor et al., 2000). The SPIN is a 17-item self-report measure that assesses the severity of three symptom domains in SAD: fear (e.g., "Parties and social events scare me"), avoidance (e.g., "I avoid having to give speeches") and physiological symptoms (e.g., "Sweating in front of people causes me distress"). Individuals respond to items based on the degree to which symptoms have been experienced in the past week. Items are measured on a 5-point Likert scale ranging from 0 (Not at all) to 4 (Extremely), with higher 
scores indicating greater levels of distress. Total SPIN scores range from 0 to 68 . A cutoff score of 19 distinguishes between individuals with and without SAD (diagnostic accuracy of 79\%), a cutoff score of 16 distinguishes between individuals with SAD and nonsocially anxious psychiatric controls (diagnostic accuracy of $80 \%$ ), and a cutoff of 15 distinguishes between individuals with SAD and individuals with no psychiatric conditions (Connor et al., 2000). Therefore, the SPIN can be a useful predictor of the likelihood that an individual will meet diagnostic criteria for SAD. The SPIN has demonstrated good psychometric properties, including good internal consistency, good test-retest reliability, construct validity, convergent validity, discriminant validity, and sensitivity to treatment effects (Antony, Coons, McCabe, Ashbaugh, \& Swinson, 2006; Connor et al., 2000). Cronbach's alpha in the present sample was .95, indicating excellent internal consistency.

Generalized Anxiety Disorder Questionnaire (GAD-Q-IV; Newman et al., 2002). The GAD-Q-IV is a widely used self-report measure that evaluates the presence of each DSM-IV diagnostic criterion of GAD (Rodebaugh, Holaway, \& Heimberg, 2008), including the occurrence of excessive and uncontrollable worry, topics and duration of worry, associated symptoms (e.g., restlessness, muscle tension), and the degree of impairment and distress caused by these symptoms. Despite a number of proposed revisions for DSM-5-defined GAD (Andrews et al., 2010), only one minor change was made from DSM-IV to DSM-5 (i.e., elimination of the hierarchical rule that GAD cannot occur exclusively in the context of a mood disorder, psychotic disorder, pervasive developmental disorder, or posttraumatic stress disorder), justifying the use of a measure based on DSM-IV diagnostic criteria. The GAD-Q-IV consists of 9 items with a range of response formats, including yes/no questions, checklists, fill-in-the-blank responses, and 
9-point Likert scales ranging from 0 (None) to 8 (Very Severely) to assess levels of symptomrelated distress and interference.

The GAD-Q-IV can be used as a measure of symptom severity or as a diagnostic screening device. Item responses are summed to yield total scores ranging from 0-13 with higher scores indicating greater symptom severity. Alternatively, a cutoff score of 7.67 can be used to predict the likelihood that an individual's symptoms will meet diagnostic criteria for GAD. A recent study found that this cutoff score achieved a better balance between diagnostic sensitivity ( $85 \%$ of individuals with GAD were predicted to have GAD) and specificity ( $74 \%$ of individuals without GAD were predicted to not have GAD) than a previously derived cutoff score of 5.7 (Moore, Anderson, Barnes, Haigh, \& Fresco, 2014), which yielded better diagnostic sensitivity (90\%), but poorer specificity (66\%). The GAD-Q-IV has demonstrated good psychometric properties, including good test-retest reliability, and good convergent and discriminant validity (Newman et al., 2000; Robinson, Klenck, \& Norton, 2010). Cronbach's alpha in the present sample was .93, indicating excellent internal consistency.

Penn State Worry Questionnaire (PSWQ; Meyer, Miller, Metzger, \& Borkovec, 1990). The PSWQ is 16-item self-report measure that assesses the frequency and intensity of trait-level worry (e.g., "I am always worrying about something"). Items are rated on a 5-point Likert scale ranging from 1 (Not at all typical of me) to 5 (Very typical of me), with higher scores corresponding to greater levels of worry. Total scores range from 16-80. Previous research has demonstrated the utility of the PSWQ as a screening measure using a cutoff score of 45 to distinguish between individuals with GAD and nonanxious, nondepressed individuals with a high degree of sensitivity (0.99) and specificity (0.98) (Behar, Alcaine, Zuellig, \& Borkovec, 2003). In a sample of patients seeking treatment for anxiety, a higher cutoff score of 65 
distinguished between individuals with GAD and individuals with SAD with a good degree of sensitivity (.68) and specificity (.65) (Fresco, Mennin, Heimberg, \& Turk, 2003). The PSWQ has demonstrated strong psychometric properties, including excellent internal consistency, good testretest reliability, convergent, discriminant, criterion validity, and sensitivity to treatment effects (Meyer et al., 1990). Cronbach's alpha in the present sample was .95, indicating excellent internal consistency.

Depression Anxiety Stress Scales, 21-item version (DASS-21; Lovibond \& Lovibond, 1995). The DASS-21 is a 21 -item self-report measure used to evaluate symptoms of depression, anxiety, and stress/distress/tension experienced in the past week (Osman et al., 2012). It consists of three subscales each with seven items: Depression (DASS-D; e.g., "I couldn't seem to experience any positive feeling at all"), Anxiety (DASS-A; e.g., "I experienced trembling (e.g., in the hands)"), and Stress (DASS-S; e.g., "I found it hard to wind down"). Items are measured on a 4-point Likert scale ranging from 0 (Did not apply to me at all) to 3 (Applied to me very much, or most of the time), with higher scores indicating greater severity or frequency. Items assessing depression reflect symptoms of low mood, hopelessness, and worthlessness, items assessing anxiety focus on the physiological symptoms of anxiety, and items assessing stress reflect general distress and tension, which are shared features of depression and anxiety (Antony, Bieling, Cox, Enns, \& Swinson, 1998; Clark \& Watson, 1991). Only the DASS-D subscale was used in the current study in order to measure depressive symptom severity (on a continuum, rather than as a diagnostic tool). The DASS-21 has demonstrated strong psychometric properties, including good internal consistency and concurrent validity (Antony et al., 1998), and sensitivity to treatment effects (Page, Hooke, \& Morrison, 2007). Cronbach's alpha for the DASS-D subscale in the present sample was .89 . 
Intolerance of Uncertainty Scale (IUS; Buhr \& Dugas, 2002). The IUS is a 27-item selfreport measure that assesses IU, which is the excessive tendency to respond negatively to uncertainty and its perceived consequences, regardless of its actual probability (Dugas, Gagnon, Ladouceur, \& Freeston, 1998b; Sexton \& Dugas, 2009). The IUS consists of two factors: 1) "Uncertainty has negative behavioural and self-referent implications" (IU-1; e.g., "Being uncertain means that a person is disorganized") and 2) "Uncertainty is unfair and spoils everything" (IU-2; e.g., "It's unfair not having any guarantees in life"), both of which correlate highly with pathological worry (Sexton \& Dugas, 2009). Items are measured on a 5-point Likert scale ranging from 1 (Not at all characteristic of me) to 5 (Extremely characteristic of me), with higher scores corresponding to greater IU. Total scores can be calculated by summing the responses for all items, or two scores can be obtained by summing the responses for Factor 1 (15 items) and Factor 2 (12 items). In the current study, total IUS scores rather than two factor scores were calculated. The IUS has demonstrated strong psychometric properties, including excellent internal consistency and test-retest reliability, and good convergent and discriminant validity (Buhr \& Dugas, 2002; Sexton \& Dugas, 2009). Cronbach's alpha in the present sample was .95, indicating high internal consistency.

State-Trait Inventory for Cognitive and Somatic Anxiety (STICSA; Ree, French, MacLeod, \& Locke, 2008). The STICSA is a 21-item self-report measure that assesses cognitive (e.g., worry, lack of concentration) and somatic (e.g., sweating, muscle tension) dimensions of state and trait anxiety. State anxiety refers to transient symptoms of anxiety that are experienced in the moment, whereas trait anxiety refers to a dispositional tendency to experience anxiety most of the time (Spielberger, 1983). For the purposes of the current study, only the STICSATrait scale was administered to participants. This scale consists of 10 items assessing cognitive 
symptoms of anxiety (e.g., "I think the worst will happen") and 11 items assessing somatic symptoms of anxiety (e.g., "My heart beats fast"). Participants are instructed to rate, in general, how often each item applies to them on a 4-point Likert scale ranging from 1 (Almost never) to 4 (Almost always), with higher scores indicating greater trait anxiety. Because of its recent development, there is limited research on the psychometric properties of the STICSA. However, the research that does exist has indicated strong psychometric properties of each subscale, including excellent internal consistency, and good construct, convergent, and discriminant validity (Grös, Antony, Simms, \& McCabe, 2007; Grös, Simms, \& Antony, 2010; Ree et al., 2008). Cronbach's alphas in this sample was . 92 , indicating excellent internal consistency. Brief Fear of Negative Evaluation Scale (BFNE-S; Leary, 1983). The BFNE is a 12-item self-report measure that assesses the degree to which individuals fear negative evaluation from others (e.g., "I am afraid that people will find fault with me"). Items are measured on a 5-point Likert scale ranging from 1 (Not at all characteristic of me) to 5 (Extremely characteristic of $m e$ ), with higher scores corresponding to greater fear of negative evaluation. More recent research, however, has suggested excluding the four reverse-worded items from total score calculations, as these items appear to be less representative of fear of negative evaluation and social anxiety compared to the eight straightforwardly-phrased items in both undergraduate samples (Rodebaugh et al., 2004) and clinical samples (Weeks et al., 2005). The BFNE has demonstrated good psychometric properties, including high internal consistency, and factorial, construct, convergent, and discriminant validity (Rodebaugh et al., 2004; Weeks et al., 2005; Weeks, Heimberg, \& Rodebaugh, 2007). Cronbach's alpha in the present sample was .94, indicating excellent internal consistency. 
Fear of Positive Evaluation Scale (FPES; Weeks et al., 2008). The FPES is a 10-item self-report measure that assesses the degree to which individuals fear being positively evaluated by people that they "do not know very well” (e.g., "I feel uneasy when I receive praise from authority figures"). Participants are instructed to rate the degree to which each item is characteristic of them. Items are rated on a 10-point Likert scale ranging from 0 (Not at all) to 9 (Very true), with higher scores corresponding to greater fear of positive evaluation. Two items (Items 5 and 10) are reverse-worded to decrease individuals' biased tendency to positively endorse all items. Total scores, however, do not include these reverse-worded items. The FPES has demonstrated good internal consistency, good test-retest reliability over a 5-week period, as well as factorial, construct, convergent, and discriminant validity (Weeks et al., 2008). In terms of construct validity, Weeks and colleagues (2008) found that fear of positive evaluation and fear of negative evaluation are related, but distinct constructs, and that fear of positive evaluation accounted for a significant amount of variance in social interaction anxiety over and above the variance already accounted for by fear of negative evaluation. Cronbach's alpha in the present sample was .88, indicating high internal consistency.

Positive Feedback Seeking Frequency Questionnaire (PFSF-Q; Wilson, 2014). The PFSF-Q (Appendix D), which was developed for the current study, is a 25-item self-report measure that assesses the frequency with which individuals seek positive feedback or reassurance across a wide range of topics/domains (e.g., social competence, personal safety, intelligence) and from different sources (e.g., romantic partner, family member, websites). The topics/domains of feedback seeking were chosen to reflect topics assumed to be the most characteristic of social anxiety (e.g., social/evaluative domains) or generalized worry (e.g., general safety/security domains). Prior to data collection, these items were pilot tested on a group 
of 11 graduate students who conduct research in the area of anxiety and related disorders. Each student rated the extent to which each item reflects sources of fear and anxiety in socially anxious individuals versus individuals with high generalized worry. More than $75 \%$ of raters had to agree that a given item was more characteristic of either social anxiety or generalized worry for it to be considered a social/evaluative threat or a general threat, respectively. The following topics were identified as representing social/evaluative threats (i.e., topics rated as more characteristic of social anxiety): social skills or social competence, physical appearance, and signs of anxiety (e.g., sweating, shaking, blushing, stuttering). The following topics were identified as representing more general threats (i.e., topics rated as more characteristic of generalized worry): personal abilities (e.g., work, parenting, housekeeping, arts, athletics etc.), life achievements (e.g., career, education etc.), decision-making, personal health or well being, health or well being of others, personal safety, safety of others, and financial security. Fewer than $75 \%$ of raters agreed that the following topics were more characteristic of either social anxiety or generalized anxiety: Level of performance or accuracy in a specific situation, quality or security of interpersonal relationships, self worth or lovability, and intelligence. These topics were retained in the PFSF-Q and classified as “other," as they may represent topics that are characteristic of both social anxiety and generalized worry or neither.

Scores can be calculated for the three subscales (which correspond to the graduate students' ratings): PFSF-Q-Social subscale, PFSF-Q-General subscale, and PFSF-Q-Other subscale. A full scale (PFSF-Q-Total) score can also be calculated. Items are measured on 5point Likert scale, ranging from 0 (Never) to 4 (Very Often), with higher scores indicating more frequent PFS. This self-report measure was developed to assess a general tendency to seek positive feedback or reassurance. As outlined by Antony and colleagues (2005), many factors 
aside from actual behaviour frequency may account for the number of completed diaries, including increased recognition of behaviours, whether the situation enabled diary recording, forgetfulness, fatigue, and additional practical barriers. The aim of developing the PFSF-Q was to remedy this issue by providing a more trait-like measure of how often individuals, in general, seek positive feedback or reassurance about a range of topics and from different sources. Given its recent development, an examination of its psychometric properties is required. Cronbach's alphas in the present sample were .96 for items assessing topics/themes, indicating excellent internal consistency, and .72 for items assessing sources, indicating adequate internal consistency.

Negative Feedback Seeking Frequency Questionnaire (NFSF-Q; Wilson, 2014). The NFSF-Q (Appendix E), which was also developed for the current study, is a 25-item self-report measure that assesses the frequency with which individuals seek negative information about themselves across a wide range of topics/domains and sources (Appendix D). The items are identical to those included in the PFSF-Q (described earlier) and are measured on 5-point Likert scale, ranging from 0 (Never) to 4 (Very Often), with higher scores indicating more frequent NFS. Similar to the PFSF-Q, the NFSF-Q has three subscales (NFSF-Q-Social, NFSF-QGeneral, NFSF-Q-Other) and a full scale (NFSF-Q-Full). This measure was developed primarily to assess a general tendency to seek feedback, given the previously discussed concerns about the number of completed diaries failing to accurately reflect the frequency of actual behaviours (Antony et al., 2005). Cronbach's alphas in the present sample were .92 for items assessing topics/themes, indicating excellent internal consistency, and .80 for items assessing sources, indicating good internal consistency. 
Depressive Interpersonal Relationships Inventory-Reassurance-Seeking Subscale (DIRIRS; Joiner, Alfano, \& Metalsky, 1992). The DIRI-RS is a 4-item self-report measure of ERS centering on themes of worth and lovability, and the perceived reactions of close others to this behaviour. The four items of the DIRI-RS are derived from the original 24-item DIRI (Coyne, 1976), which comprises three subscales: Reassurance-Seeking, General Dependency, and Doubt in the Sincerity of Others. Given the purposes of the current study, responses to the four items comprising the Reassurance-Seeking subscale (e.g., "Do you find yourself often asking the people you feel close to how they truly feel about you?") were collected and analyzed. Items are rated on a 7-point Likert scale ranging from 1 (Not At All) to 7 (Very Much), with higher scores indicating higher reassurance seeking (or positive feedback seeking) tendencies. Total DIRI-RS scores are obtained by calculating a mean score across the four items. The criterion and construct validity of the DIRI-RS have been supported by previous research (Joiner et al., 1992). Cronbach's alpha in the present sample was .90 , indicating excellent internal consistency. Reassurance Seeking Scale (RSS; Rector, Kamkar, Cassin, Ayeasrt, \& Laposa, 2011). The RSS is a recently developed 30-item self-report questionnaire that assesses a range of factors that may underlie ERS in anxiety-based problems. The RSS consists of three factors: 1) RSSDecision-Making, which comprises 13 items that assess the need to seek reassurance about decision-making (e.g., ERS "Prior to making a decision"), 2) RSS-Social Attachment, which comprises eight items that assess the need to seek reassurance about interpersonal attachment and relationship security (e.g., ERS to determine "whether you have received a negative evaluation"), and 3) RSS-General Threat, which comprises nine items that assess the need to seek reassurance about perceived general threats (e.g., ERS to determine "whether you are safe"). All items are measured on a 5-point Likert scale ranging from 1 (Not at all) to 5 
(Extremely), with higher scores corresponding to more frequent ERS. The RSS has demonstrated good internal consistency, as well as construct and convergent validity (Rector et al., 2011). Cronbach's alphas in the present sample were .91 for RSS-Decision-Making, .90 for RSS-Social Attachment, and .86 for RSS-General Threat, indicating high internal consistency.

Feedback Seeking Questionnaire (FSQ: Swann et al., 1992a). The FSQ is a 30-item selfreport measure that assesses feedback seeking preferences within the following five domains: social abilities, intellectual abilities, artistic/musical abilities, athletic abilities, and physical appearance. Participants are instructed to select two questions (out of six questions) from each domain that they would hypothetically want a close other to answer about them in order to "learn more about themselves." Three of the six questions in each domain are phrased in positive terms (e.g., "What is some evidence you have seen that [participant's name] has good social skills?"), whereas the other three questions are phrased in negative terms (e.g., "What is some evidence you have seen that [participant's name] doesn't have very good social skills?"). Total scores were obtained by adding the number of selected questions that were negatively framed, with higher scores reflecting a greater preference for negative feedback (Note: the FSQ was not designed to measure preference for positive feedback). The FSQ has demonstrated satisfactory psychometric properties, including adequate internal consistency and construct validity (e.g., Joiner, Alfano, \& Metalsky, 1993; Joiner, Katz, \& Lew, 1997). Feedback Seeking Diary (FSD; Wilson, 2014). The FSD, which was developed for the current study, was adapted from the Rochester Social Comparison Diary (RSCD; Wheeler \& Miyake, 1992), designed for recording instances of social comparisons in day-to-day life. Modified versions of the RSCD have been successfully implemented in previous research on social comparison processes in social anxiety (Antony et al., 2005). The FSD was designed for 
recording episodes of PFS and NFS each time they occur. As mentioned earlier, the term PFS was chosen over ERS for the purposes of clarity and to emphasize that this form of feedback seeking can be conceptualized as the opposite of NFS. Whereas PFS is believed to reflect a desire for positive self-relevant information, NFS is believed to be motivated by a desire for negative self-relevant information. For each instance of PFS or NFS, participants were asked to record: (a) their name, the date, time of recording, and time of feedback seeking, (b) the type of feedback seeking behaviour (PFS or NFS), (c) the triggers of feedback seeking (d) the topics/domains on which their feedback seeking focused (e.g., security of relationships, personal safety), (e) the source of their feedback seeking (i.e., from whom feedback was sought) (e.g., romantic partner, professional/authority figure), (f) how they felt just before seeking feedback and just after receiving feedback (e.g., anxious, uncertain, ashamed), all of which are measured on a 7-point Likert scale ranging from 0 (Not at all) to 6 (Extremely) $(\mathrm{g})$ the extent to which received feedback is trusted, measured on a 7-point Likert scale ranging from 0 (Not at all) to 6 (Very much), with higher scores corresponding to greater perceived trust in received feedback, (h) the type of feedback (negative, positive, or neutral) that was expected, hoped for, and received (which are all separate questions in the FSD), (i) the reasons for seeking feedback (e.g., to ensure loved ones are safe), and (j) the reasons for terminating feedback seeking (e.g., to avoid interpersonal conflict) (Appendix C). The topics/domains included in the FSD are identical to those included in the PFSF-Q and the NFSF-Q (described earlier), and were therefore categorized as topics reflecting social/evaluative concerns (e.g., signs of anxiety), general concerns (e.g., safety of others), or "other" concerns (e.g., quality or security of interpersonal relationships). In addition, when participants completed the FSDs, there was no limit on the number of feedback topics/domains that could be selected. For instance, an individual who 
sought feedback about his or her performance during a presentation could select both "level of performance or accuracy in specific situation" and "signs of anxiety (e.g., sweating, shaking, blushing, stuttering)" as the topics/domains on which their feedback seeking focused. Of note, responses to how much received feedback was trusted were not analyzed because predictions about trust were not central to the purpose of the current study.

On the second page of the FSD were brief descriptions of PFS and NFS to help participants accurately identify their feedback seeking behaviours. A brief description of neutral feedback (i.e., feedback that is neither clearly positive nor clearly negative) was also provided. The author reviewed each definition with participants and provided an example of each behaviour. Previous research found that individuals' reports of their own reassurance seeking moderately and positively correlated with others' reports of the individuals' reassurance seeking, suggesting that individuals have some insight into their reassurance seeking and are therefore able to identify this behaviour (see Evraire \& Dozois, 2011). Further, researchers have suggested that the desire to self-enhance (via seeking positive feedback) is a more automatic process than the desire to self-verify (via seeking negative feedback). Self-enhancement requires the individual to simply decide whether received feedback is favourable or not, whereas selfverification requires the individual to identify their self-beliefs and then determine whether received feedback is consistent with these beliefs. Therefore, the process of self-verification is believed to require greater cognitive resources (e.g., greater awareness of negative self-beliefs) than does self-enhancement, suggesting that episodes of PFS may be easier to identify and therefore easier to report compared to episodes of NFS. 


\section{Procedure}

This study took place in the Anxiety Research and Treatment Lab at Ryerson University. Individuals who responded to recruitment advertisements completed the MINI over the telephone to determine their eligibility. Individuals were asked additional questions that assessed for disorders not included in the MINI (e.g., specific phobia, body dysmorphic disorder) to ensure that SAD or GAD was the principal diagnosis, or that individuals had no lifetime history of mental health difficulties. Following completion of the MINI, eligible individuals who agreed to participate in the study were scheduled to come into the lab for their first laboratory session. Upon arrival at the lab, participants were provided with a Letter of Information and Consent Form, which outlined the tasks involved in the study, limits of confidentiality, and voluntary participation. All participants provided written informed consent.

Next, participants provided basic demographic information (e.g., age, gender, ethnicity) and completed the following questionnaires (see Measures for detailed descriptions): SPIN, GAD-Q-IV, PSWQ, DASS-21, IUS, STICSA, FPES, BFNE, PFSF-Q, NFSF-Q, DIRI-RS-RS, RSS, and FSQ. Participants were then shown a copy of the FSD (see Measures), which they were asked to fill out each time they engaged in either PFS or NFS over the following 2 weeks. Each diary record included definitions of positive and negative feedback seeking to facilitate participants in accurately identifying each of these behaviours. The author of the current study reviewed each item of the diary record in detail while addressing participants' questions or concerns to ensure that they understood the diary protocol. Before leaving the lab, participants were provided with a stack of hard-copy diary records to complete in the event that they were unable to access the electronic diary records. 
During the 2 weeks of diary recording, participants were sent a daily email reminder with a link to an electronic version of the diary record, which was developed using Qualtrics Online Survey Software. The purpose of sending a daily email reminder with electronic diary records attached was to prompt participants to accurately complete their diaries on a daily basis. Following the 2 weeks of diary recording, all participants returned to the lab at which point any completed hard copy diary records were submitted and participants' questions or concerns about the study were addressed. Participants were asked to report their compliance ratings, which was the percentage of feedback seeking behaviours captured in the hard copy and/or online FSDs. For example, if a participant completed 10 FSDs, but reported capturing $50 \%$ of their feedback seeking behaviours, then the estimated frequency (accounting for reported compliance) would be 20 feedback seeking episodes. This specific protocol for measuring participants' compliance has been used in previous diary studies conducted in the Anxiety Research and Treatment Lab at Ryerson University (McCabe-Bennett, 2014). Finally, all participants were debriefed and compensated 30 dollars for completing the study. 


\section{Results}

All questionnaire and diary data were checked for missing values, outliers, and normality.

Missing data. Missing questionnaire values were replaced with the individual's mean score on the respective scale or subscale. Diary records that had more than $20 \%$ missing data (due to noncompliance to the diary protocol) were excluded from analyses, and participants who had more than $20 \%$ of their diary records removed were excluded from analyses. Twelve diary records were removed because more than $20 \%$ of the data were missing. One diary record was removed because the individual reported receiving feedback without actually seeking feedback. Another diary record was removed because the individual indicated considering seeking feedback, but did not actually engage in this behaviour. Two participants were excluded because more than $20 \%$ of their total diary records were removed due to missing data. This resulted in a sample size of 66 and a total of 522 diary records (before excluding outliers).

Outliers. Outliers were defined as values greater than 2.5 standard deviations above or below the mean of a particular scale or subscale. One participant was an outlier on the DASS-D and the NFSF-Q-Social, one participant was on outlier on the NFSF-Q-General, and one participant was on outlier on the FSQ. Another participant was an outlier on the frequency of PFS diaries and overall diaries, and two participants were outliers on the frequency of NFS diaries. These outliers were excluded from analyses, resulting in a final sample size of 61 and a total of 461 diary records. See Table 2 for descriptive statistics for frequency of PFS, NFS, and overall feedback seeking (based on diary records) stratified by group.

Normality. Kolmogorov-Smirnov (K-S) tests were conducted to determine whether the distributions of questionnaire and diary data within each group significantly deviated from normality. The GAD-Q- scores, $D(20)=0.20, p<.05$, SPIN scores, $D(20)=0.20, p<.05$, 
PSWQ scores, $D(20)=0.21, p<.05$, and IUS scores, $D(20)=0.20, p<.05$, of individuals with GAD were significantly nonnormal, such that all distributions were negatively skewed (with the exception of IUS scores). The healthy comparison group's GAD-Q scores, $D(20)=0.30, p<$ .001 , DASS-D scores, $D(20)=0.27, p<.001$, RSS Social- Attachment scores, $D(20)=0.20, p<$ .05 , DIRI-RS scores, $D(20)=0.23, p<.01$, PFSF-Q-Social scores, $D(20)=0.24, p<.01$, NFSFQ-Social scores, $D(20)=0.30, p<.001$, NFSF-Q-General scores, $D(20)=0.30, p<.001$, and FPES scores, $D(20)=0.21, p<.05$, were significantly nonnormal, such that all distributions were positively skewed. The FSQ scores of the GAD group and the healthy comparison group were positively skewed, and the scores of the SAD group were platykurtic.

Further, the frequency of PFS diaries for the SAD and GAD groups were significantly nonnormal, $D(21)=0.23, p<.01$ and $D(20)=0.21, p<.05$, respectively; however, the skewness and kurtosis values were not significant (all $z$-scores $<1.96$ ). All three groups' scores on frequency of NFS diaries were significantly nonnormal (all $p s<.01$ ); however, only the SAD and healthy comparison groups had significant positive skewness ( $z$-scores $>1.96)$. The total number of diaries (PFS and NFS diaries combined) were significantly nonnormal for individuals with GAD, $D(20)=0.21, p<.05$; however, this distribution had neither significant skewness nor kurtosis $(z$-scores $<1.96)$.

The distributions of the following topics/domains and sources were significantly nonnormal (all $p s<.05)$ : PFS-Social in the healthy comparison group, which was significantly positively skewed and leptokurtic (z-scores $>1.96)$; NFS-Social, NFS-General, and NFS-Other for all groups (all $p s<.05$ ), all of which were significantly positively skewed with the exception of NFS-General in the GAD group ( $z$-score $<1.96)$; overall feedback seeking about social/evaluative and "other" concerns in the healthy comparison group; however, only 
social/evaluative concerns were significantly positively skewed and significantly leptokurtic; and number of NFS sources for all groups, with all distributions significantly positively skewed.

The following triggers, functions, and termination criteria were significantly nonnormal (all $p s<.05$ ); PFS triggered by general concerns in the healthy comparison group; PFS triggered by "other" concerns in all groups; NFS triggered by social/evaluative and general concerns in all groups; NFS triggered by "other" concerns for individuals with GAD and healthy individuals; overall feedback seeking triggered by "other" concerns for all groups; PFS aimed at reducing general concerns in the healthy comparison group; PFS aimed at reducing "other" concerns in all groups; NFS designed to avoid appearing "too good" in the healthy comparison group; termination of PFS related to social/evaluative concerns in the two clinical groups; termination of PFS related to general concerns in the healthy comparison and SAD groups; termination of PFS related to "other" concerns in healthy individuals; and termination of NFS related to confirmation of negative self-views in all groups.

Positive affect before engaging in NFS was significantly nonnormal for individuals with GAD, $D(13)=0.27, p<.05$, such that the distribution was significantly positively skewed $(z-$ score $>1.96$ ). Negative affect after receiving positive feedback was significantly nonnormal for individuals with GAD, $D(7)=0.31, p<.05$, such that the distribution was positively skewed, albeit not to a significant degree $(z$-score $<1.96)$. Positive affect following the receipt of negative feedback was significantly nonnormal for individuals with $\mathrm{SAD}, D(7)=0.32, p<.05$, and individuals with $\mathrm{GAD}, D(7)=0.40, p<.01$, such that these distributions were positively skewed however, albeit not to a significant degree $(z$-scores $<1.96)$.

Preliminary Analyses. A series of analyses of variance (ANOVA) tests and Pearson Chi-square tests were conducted to evaluate potential group differences on gender, age, ethnicity, 
relationship status, current program enrolment, type of program, level of education, employment status, and self-reported compliance ratings. There were no significant group differences on any of these variables: gender, $\chi^{2}(4)=4.12, p>.05$, age, $F(2,57)=1.35, p>.05$, ethnicity, $\chi^{2}(10)=$ $10.78, p>.05$, relationship status, $\chi^{2}(8)=5.68, p>.05$, program enrolment, $\chi^{2}(2)=.12, p>$ .05 , type of program, $\chi^{2}(4)=2.06, p>.05$, level of education, $\chi^{2}(8)=7.26, p>.05$, employment status, $\chi^{2}(4)=.76, p>.05$, and compliance ratings, $F(2,58)=1.92, p>.05$. The demographic characteristics of participants stratified by group are presented in Table 1.

Next, a series of ANOVAs and Kruskal-Wallis tests were conducted to evaluate potential group differences on worry (measured by the GAD-Q-IV and PSWQ), social anxiety (measured by the SPIN), depressive symptoms (measured by the DASS-D), trait anxiety (measured by the STICSA), IU (measured by the IUS), and fear of negative and positive evaluation (measured by the BFNE and FPES, respectively).

A Kruskal-Wallis test indicated a significant group difference on GAD-Q-IV scores, $H(2)$ $=33.85, p<.001$, such that the SAD group $(M d n=6.50)$ and the GAD group $(M d n=10.09)$ had significantly greater GAD-Q-IV scores than the healthy comparison group $(M d n=.67), U=$ $34.50, z=-4.60, p<.001, r=-0.59$, and $U=8.50, z=-5.21, p<.001, r=-0.67$, respectively. There was no significant difference between the two clinical groups, $U=143.50, z=-1.74, p>$ $.05, r=-.27$ (however, $r=.27$ represents a medium effect). The GAD-Q-IV can also be used as a diagnostic screening device to predict the likelihood that an individual's symptoms will meet diagnostic criteria for GAD. The recommended cutoff score is 7.67 (Moore et al., 2014). Seventeen individuals diagnosed with GAD (85\%), nine individuals diagnosed with SAD (42.90\%), and one healthy individual (5\%) scored at least 7.67 on the GAD-Q-IV. A Chi-square test indicated a significant association between group and whether individuals were above or 
below the 7.67 cutoff score, $\chi^{2}(2)=25.97, p<.001$, Cramer's $V=.65$. Based on the odds ratio, individuals with GAD were 7.56 times more likely to score above the cutoff score than individuals with SAD and 113.40 times more likely than healthy individuals. The odds of individuals with SAD scoring above the cutoff score were 15 times higher than those of healthy individuals.

Following a square root transformation, the PSWQ scores of individuals with GAD were no longer significantly nonnormal, $D(20)=0.15, p>.05$. Therefore an ANOVA was conducted, indicating a significant group difference on PSWQ scores, $F(2,58)=36.24, p<.001, \omega^{2}=0.54$. Bonferroni tests indicated that the SAD group $(M=62.64, S D=8.36)$ had significantly greater PSWQ scores than the healthy comparison group $(M=37.05, S D=11.23), p<.05, r=.80$. The GAD group $(M=64.43, S D=10.75)$ also had significantly greater PSWQ scores than the healthy comparison group $(M=37.05, S D=11.23), p<.05, r=.79$. However, there was no significant difference between the two clinical groups, $p>.05, r=.10$. As mentioned earlier, the PSWQ has a recommended cutoff score of 65 that was found to distinguish between individuals with GAD and individuals with SAD with a good degree of sensitivity and specificity (Fresco et al., 2003). Nine individuals diagnosed with GAD (45\%), nine individuals diagnosed with SAD (42.90\%), and no healthy individuals $(0 \%)$ scored at least 65 on the PSWQ. A Chi-square test indicated a significant association between group and whether individuals were above or below the 65 cutoff score, $\chi^{2}(2)=12.50, \mathrm{p}<.01$, Cramer's $\mathrm{V}=.45$. Based on the odds ratio, individuals with GAD were 1.09 times more likely to score above the cutoff score than individuals with SAD. Odds ratios could not be calculated for the healthy group because none of these individuals scored above the cutoff score. 
A Kruskal-Wallis test indicated a significant group difference on SPIN scores, $H(2)=$ 45.94, $p<.001$, such that the SAD group $(M d n=43.00)$ had significantly greater SPIN scores than the healthy comparison group $(M d n=6.50), U=.00, z=-5.48, p<.001, r=-.70$. The GAD group $(M d n=30.00)$ also had significantly greater SPIN scores than the healthy comparison group $(M d n=6.50), U=20.00, z=-4.87, p<.001, r=-.62$, and the SAD group $(M d n=43.00)$ had significantly higher SPIN scores than the GAD group $(M d n=30.00), U=$ $40.50, z=-4.43, p<.001, r=-.57$. Similar to the GAD-Q-IV, the SPIN can be a useful predictor of the likelihood that diagnostic criteria will be met for SAD (Connor et al., 2000). The recommended cutoff score for distinguishing between individuals with and without SAD is 19 . Twenty-one individuals diagnosed with SAD (100\%), 17 individuals diagnosed with GAD (85\%), and two healthy individuals (10\%) scored above 19 on the SPIN. A Chi-square test indicated a significant association between group and whether individuals were above or below the cutoff score, $\chi^{2}(2)=41.73, p<.001$, Cramer's $\mathrm{V}=.83$. Based on the odds ratio, individuals with SAD were 3.70 times more likely to score above the SPIN cutoff score than individuals with GAD and 190.91 times more likely than healthy individuals. The odds of individuals with GAD scoring above the cutoff score were 51.55 times higher than healthy individuals.

A Kruskall-Wallis test revealed a significant group difference on DASS-D scores, $H(2)=$ $24.89, p<.001$, such that the SAD group $(M d n=12.00)$ and GAD group $(M d n=19.00)$ had significantly greater DASS-D scores than the healthy group ( $M d n=2.00), U=82.00, z=-3.37$, $p<.01, r=-.43$, and $U=25.50, z=-4.76, p<.001, r=-.61$, respectively. The two clinical groups did not significantly differ $(p>.05), r=.30$ (however, $r=.30$ represents a medium effect). Similarly, there was a significant group difference on STICSA scores, Welch's $F(2)=$ $81.93, p<.001, \omega^{2}=.59$, such that the SAD group $(M=44.76, S D=10.59)$ and GAD group $(M$ 
$=50.30, S D=6.38)$ had significantly greater STICSA scores than the healthy group $(M=28.10$, $S D=4.89)(p s<.05), r=.70$ and $r=.90$, respectively. There was no significant difference between the two clinical groups $(p>.05), r=.31$ (however, $r=.31$ represents a medium effect).

A Kruskal-Wallis test indicated a significant group difference on IUS scores, $H(2)=$ $26.11, p<.001$, such that the SAD group $(M d n=85.00)$ and GAD group $(M d n=82.50)$ had significantly greater IUS scores than the healthy group ( $M d n=49.00), U=42.50, z=-4.37, p<$ $.001, r=-.68, U=35.00, z=-4.47, p<.001, r=-.71$, respectively. There was no significant difference in IUS scores between the two clinical groups, $U=209.00, z=-.03, p>.05, r=-.004$.

There were significant group differences in BFNE scores, $F(2,58)=43.22, p<.001, \omega^{2}$ $=.58$, such that the $\mathrm{SAD}$ group $(M=29.57, S D=7.17)$ and $\mathrm{GAD}$ group $(M=31.85, S D=4.65)$ had significantly greater BFNE scores than the healthy group $(M=15.10, S D=6.44)(p s<.05)$, $r=.74$, and $r=.84$, respectively. There was no significant difference between the two clinical groups, $p>.05, r=.19$. Similarly, there was a significant group difference in FPES sores, $H(2)=$ $11.70 p<.01$, such that the SAD group $(M d n=37.00)$ and GAD group $(M d n=34.50)$ had significantly greater FPES scores than the healthy group $(M d n=12.00), U=93.50, z=-3.04, p$ $<.01, r=-.39$, and $U=96.50, z=-2.80, p<.01, r=-.36$, respectively. There was no significant difference in FPES scores between the two clinical groups, $U=184.50, z=-.67, p>.05, r=-$ 0.10 . See Table 3 for self-report questionnaire scores stratified by group.

In addition, as shown in Table 2, the frequency of feedback seeking over a 2-week period was quite low. Although no predictions were made about how frequently each group would engage in PFS and NFS over 2 weeks, the reported frequency was lower than expected given that these behaviours have been consistently described as "excessive" in the literature (see Discussion for more detail). In the SAD group, the mean frequency of PFS was $5.67(S D=3.67)$ and the 
mean frequency of NFS was $.90(S D=1.22)$. In the GAD group, the mean frequency of PFS was $7.90(S D=4.54)$ and the mean frequency of NFS was $1.35(S D=1.22)$. In the healthy group, the mean frequency of PFS was $5.85(S D=4.72)$ and the mean frequency of NFS was $1.05(S D=$ 1.47). During the 2-week period, PFS was reported significantly more frequently than NFS in individuals with $\mathrm{SAD}, t(20)=6.28, p<.001, r=.81$, individuals with $\mathrm{GAD}, t(19)=6.14, p<$ $.001, r=.82$, and healthy individuals, $t(19)=5.04, p<.001, r=.76$. Further, the frequency of PFS and NFS combined (i.e., overall feedback seeking) and adjusted for participants' selfreported compliance ratings was $8.81(S D=5.69)$ for the $\mathrm{SAD}$ group, $13.30(S D=7.62)$ for the GAD group, and $8.40(S D=6.46)$ for the healthy group, which seemed low for an ostensibly problematic or "excessive" behaviour.

\section{Hypothesis 1. Individuals with SAD and individuals with GAD will engage in} significantly more feedback seeking than individuals in the healthy comparison group. To determine whether there were significant group differences in the frequency of PFS, NFS, and overall feedback seeking (PFS and NFS combined) over a 2-week period, a series of multiple linear regressions were conducted. Because group was a categorical predictor, two dummy variables were created (with the healthy comparison group as the baseline) (Field, 2009). The first dummy variable compared the frequency of feedback seeking between the SAD group and the nonclinical comparison group and the second dummy variable compared the frequency of feedback seeking between the GAD group and the nonclinical comparison group.

In the first series of regression analyses the first two dummy variables were entered as the independent variables and frequency of feedback seeking was entered as the dependent variable. As displayed in the first row of Table 4, there were no significant group differences in the frequency of PFS, $R^{2}=.05, F(2,58)=1.66, p>.05$. As displayed in the second row of Table 4, 
there were no significant group differences in the frequency of NFS, $R^{2}=.02, F(2,58)=.60, p>$ .05. While there were no significant group differences in the frequency of PFS or NFS, paired samples t-tests indicated that PFS was significantly more frequent than NFS in individuals with $\mathrm{SAD}, t(20)=6.28, \mathrm{p}<.001, r=.81$, individuals with $\mathrm{GAD}, t(19)=6.12, \mathrm{p}<.001, r=.81$, and healthy individuals, $t(19)=5.04, \mathrm{p}<.001, r=.76$ (see Table 2 ). As displayed in the third row of Table 4, there were no significant group differences in the frequency of overall feedback seeking (based on the number of FSDs), $R^{2}=.06, F(2,58)=1.86, p>.05$.

Another regression was conducted in which overall feedback seeking adjusted for participants' reported compliance ratings was entered as the dependent variable. As displayed in the fourth row of Table 4 , the overall model was significant, $R^{2}=.10, F(2,58)=3.36, p<.05$. There was no significant difference between individuals with $\operatorname{SAD}(M=8.81, S D=5.69)$ and healthy individuals $(M=8.40, S D=6.46), \beta=.03, t(58)=.20, p>.05$. However, there was a significant difference between individuals with GAD $(M=13.30, S D=7.69)$ and healthy individuals $(M=8.40, S D=6.46), \beta=.34, t(58)=2.33, p<.05$, such that individuals with GAD engaged in significantly more overall feedback seeking (adjusted for compliance) than healthy individuals.

An additional regression was conducted in which IUS scores were entered in the first step of the model to determine whether the association between group (individuals with GAD versus healthy individuals) and overall feedback seeking was significant over and above the effect of IU. The group variable and IUS scores were standardized and multiplied together to create an interaction term, so that potential moderation could be evaluated. The group variable was entered in the second step of the model, followed by the interaction term. The overall model, which included IUS scores, the group variable, and the interaction term was significant, $R^{2}=.22, F(3$, 
$57)=5.24, p<.01$. As displayed in the fifth row of Table 4, IUS scores were significantly positively associated with frequency of overall feedback seeking, $\beta=.39, t(57)=2.63, p<.05$, such that increases in IUS scores predicted increases in feedback seeking. The group variable was no longer significantly associated with the dependent variable over and above the effect of IUS scores, $\beta=.09, t(57)=.61, p>.05$. Further, when the group variable was entered into the model before IUS scores, IU remained a significant predictor of overall feedback seeking frequency over and above the effect of group, $\beta=.39, t(57)=2.63, p<.05$. Importantly, the interaction term was significant, $\beta=.34, t(57)=2.36, p<.05$, indicating that IUS scores significantly interacted with the group variable in predicting the frequency of overall feedback seeking adjusted for compliance ratings. The same analyses were conducted to determine whether DASS-D, BFNE, FPES, and STICSA scores significantly moderated the relation between group and frequency of overall feedback seeking; however, none of these interaction terms were significant, $\beta=-.04, t(57)=-.28, p>.05, \beta=.06, t(57)=-.33, p>.05, \beta=.06, t(57)$ $=.46, p>.05$, and $\beta=.02, t(57)=.14, p>.05$, respectively.

Simple effects analyses were conducted to examine whether IUS scores significantly moderated the effect of group (individuals with GAD versus healthy individuals) on overall feedback seeking frequency. IUS scores that were at least one standard deviation above the mean were labeled as "high" $(M=103.07, S D=6.85)$ and scores that were at least one standard deviation below the mean were labeled as "low" $(M=37.23, S D=1.13)$ (Preacher, Curran, \& Bauer, 2006). Results indicated that levels of IUS scores did not significantly moderate the relation between group and feedback seeking frequency, such that there were no significant group differences in feedback seeking frequency at low levels of IU, $t(36)=-1.58, p>.05$, or high levels of IU, $t(36)=1.88, p>.05$. Additional simple effects analyses were conducted to 
examine whether group (individuals with GAD versus healthy individuals) moderated the relation between IUS scores and feedback seeking frequency. There was no significant association between IUS scores and feedback seeking frequency in the healthy group, $t(36)=$ $.13, p>.05$. However, there was a significant association between IUS scores and feedback seeking frequency in the GAD group, $t(36)=2.89, p<.01$, such that increases in IUS scores significantly predicted increases in the frequency of overall feedback seeking (adjusted for participants' self-reported compliance ratings). The moderating effect of group on the association between IUS scores and overall feedback seeking frequency is displayed in Figure 1.

Lastly, due to concerns about whether the number of completed diaries accurately reflected the actual frequency of feedback seeking behaviours (Antony et al., 2005), a series of Spearman's correlations were conducted to evaluate potential associations between the number of completed FSDs and scores on the PFSF-Q, NFSF-Q, RSS, DIRI-RS, and FSQ, all of which are valid and reliable self-report measures of feedback seeking. FSDs on PFS significantly correlated with the PFSF-Q-General subscale and the PFSF-Q-Full scale. FSDs on NFS significantly correlated with the the PFSF-Q-Social and Other subscales, the PFSF-Q-Full scale, the NFSF-Q-Social, General, and Other subscales, and the NFSF-Q-Full scale, the RSS-Decision subscale, and the DIRI-RS. Total FSDs (PFS and NFS combined) not adjusted for compliance significantly correlated with the PFSF-Q-General and Other subscales, and the PFSF-Q-Full scale. Total FSDs adjusted for compliance significantly correlated with the PFSF-Q-Social, General, and Other subscales, and the PFSF-Q-Full scale. Refer to Table 5 for a summary of these correlations.

Hypothesis 2. There will be no significant differences between individuals with SAD and individuals with GAD in feedback seeking about social/evaluative concerns, whereas 
individuals with GAD will seek significantly more feedback about general threats. Similar to the analyses performed by Antony and colleagues (2005), the topics of feedback seeking were measured by adding the number of feedback seeking episodes that focused on each topic for each participant. This sum was divided by the total number of diaries completed by each participant to yield a percentage of feedback seeking that focused on each domain. An average percentage of the items comprising each topic category (social/evaluative, general, and other) was calculated, yielding an average percentage of diaries that focused on each category.

A series of multivariate analyses of variance (MANOVA) tests were conducted to evaluate potential group differences on various topics of PFS, NFS, and overall feedback seeking. Using Pillai's Trace, there were no significant group differences on social/evaluative concerns, general concerns, or "other" concerns for PFS, $V=0.12, F(6,114)=1.26, p>.05$, NFS, $V=0.11, F(6,114)=1.11, p>.05$, or overall feedback seeking, $V=0.13, F(6,114)=$ $1.30, p>.05$. See Table 6 for a summary of mean percentages of topics of overall feedback seeking over a 2-week period (based on diary records) stratified by group.

Additional MANOVAs were conduced to evaluate potential group differences on the PFSF-Q and NFSF-Q. Using Pillai's Trace, there was a significant effect of group on PFS topics, $V=0.27, F(6,114)=2.96, p<.05$, with follow-up ANOVAs indicating significant group differences on social/evaluative concerns, $F(2,58)=8.61, p<.01$, general concerns, $F(2,58)=$ $6.50, p<.01$, and "other" concerns, $F(2,58)=5.60, p<.01$. Helmert contrasts revealed that individuals with SAD and individuals with GAD (combined) reported significantly more PFS focused on social/evaluative concerns $(M=6.34, S D=2.47)$, general concerns $(M=17.59, S D=$ 5.03), and "other" concerns $(M=9.80, S D=3.27)$ compared to healthy individuals $(M=3.55$, $S D=2.44$ for social/evaluative; $M=11.78, S D=7.36$ for general; $M=6.60, S D=4.04$ for 
"other"), $p s<.05$. There were no significant differences between the two clinical groups on PFS focused on social/evaluative concerns $(M=6.48, S D=2.23$ for the SAD group; $M=6.20, S D=$ 2.75 for the GAD group), general concerns $(M=17.29, S D=3.82$ for the SAD group; $M=$ $17.90, S D=6.15$ for the GAD group), or "other" concerns $(M=10.10, S D=2.83$ for the $\mathrm{SAD}$ group; $M=9.50, S D=3.72$ for the GAD group), $p s>.05$. Further, IUS, DASS-D, BFNE, and STICSA scores did not significantly moderate associations between group and any topics of PFS, $p s>.05$, and FPES scores did not moderate associations between group and PFS focused on social and general topics, $p s>.05$. However, there was a significant interaction between FPE and group (GAD versus healthy) in predicting PFS focused on "other" concerns (e.g., quality or security of relationships, self-worth), $\beta=-.26, t(57)=-2.10, p<.05$. FPES scores that were at least one standard deviation above the mean were labeled as "high" $(M=54.82, S D=5.63)$ and scores that were at least one standard deviation below the mean were labeled as "low" ( $M=5.07$, $S D=3.33)$. Simple effects analyses indicated that individuals with GAD reported engaging in significantly more PFS focused on "other" concerns than healthy individuals at low levels of FPE, $t(57)=2.53, p<.05$, whereas there was no significant group difference at high levels of FPE, $t(57)=-1.33, p>.05$. Additional simple effects analyses indicated that healthy individuals sought significantly more positive feedback about "other" concerns at high levels of FPE than at low levels, $t(57)=3.00, p<.01$, whereas there was no association between PFS focused on “other" concerns and levels of FPE in individuals with GAD, $t(57)=-1.38, p>.05$.

Similarly, there was a significant group effect on topics of NFS, $V=0.27, F(6,114)=$ 2.90, $p<.05$, with follow-up ANOVAs indicating significant group differences on NFS focused on social/evaluative concerns, $F(2,58)=9.81, p<.01$, and "other" concerns, $F(2,58)=7.46, p$ $<.01$. Group differences on NFS focused on "general" concerns approached significance, $F(2$, 
$58)=3.02, p=.05$. Helmert contrasts revealed that individuals with SAD and individuals with GAD (combined) reported significantly more NFS focused on social/evaluative concerns ( $M=$ 4.51, $S D=2.93)$, general concerns $(M=8.02, S D=6.25)$, and "other" concerns $(M=5.65, S D=$ 3.75) compared to healthy individuals ( $M=1.30, S D=1.89$ for social/evaluative; $M=3.80, S D$ $=6.04$ for general; $M=2.00, S D=2.69$ for "other"), $p s<.05$. There were no significant differences between the two clinical groups on NFS focused on social/evaluative concerns ( $M=$ 4.48, $S D=3.01$ for the $\mathrm{SAD}$ group; $M=4.55, S D=2.91$ for the GAD group), general concerns ( $M=7.90, S D=6.72$ for the $\mathrm{SAD}$ group; $M=8.15, S D=5.89$ for the GAD group), or "other" concerns ( $M=5.51, S D=3.71$ for the SAD group; $M=4.46, S D=3.83$ for the GAD group), $p s$ $>$.05. Further, IUS, DASS-D, FPE, FNE, and STICSA scores did not significantly moderate associations between group and topics of NFS, ps $>.05$.

Hypothesis 3: Individuals with GAD will seek feedback from a significantly greater number of sources than individuals with SAD and healthy individuals, whereas individuals with SAD will seek feedback from significantly more sources than healthy individuals. A series of MANOVAs were conducted to evaluate potential group differences in the number of different sources from which feedback was sought. The number of different sources from which positive feedback was sought across all participants ranged from one (e.g., family member) to six (e.g., family member, friend, acquaintance, oneself, professional/authority figure, and websites). There were no significant differences in the number of sources of PFS between individuals with $\operatorname{SAD}(M=2.67, S D=1.65)$, individuals with $\operatorname{GAD}(M=3.45, S D=1.57)$, and healthy individuals $(M=2.70, S D=1.89), F(2,58)=1.35, p>.05$. The number of different sources from which negative feedback was sought across all participants ranged from one (e.g., family member) to five (e.g., romantic partner, friend, acquaintance, stranger, and professional/authority 
figure). There were no significant group differences in the number of sources of NFS between individuals with $\operatorname{SAD}(M=.90, S D=1.37)$, individuals with $\operatorname{GAD}(M=1.35, S D=1.35)$, and healthy individuals $(M=.85, S D=1.09), F(2,58)=.92, p>.05$.

Next, a series of MANOVAs were conducted to evaluate potential group differences in the percentage of feedback seeking episodes in which feedback was sought from each source. The number of times that each participant sought feedback from each source was added and divided by the total number of completed diaries to yield a percentage of feedback seeking episodes in which feedback was sought from each source. For example, if a participant completed 10 PFS diary records and four of those episodes involved seeking positive feedback from family members, then $40 \%$ of the participant's positive feedback was sought from family members. Using Pillai's Trace, there were no significant group differences in the percentage of positive feedback sought from each source, $V=.35, F(16,104)=1.38, p>.05$, the percentage of negative feedback sought from each source, $V=.18, F(16,104)=.63, p>.05$, or the percentage of overall feedback sought from each source, $V=.34, F(16,104)=1.33, p>.05$.

Next, a series of repeated-measures ANOVAs were conducted to evaluate potential differences in the percentage of feedback sought from people, oneself, and sources other than people (e.g., websites, books) within each group. In the SAD group there was a significant difference between these sources, $V=.74, F(2,19)=26.38, p<.01$, such that these individuals sought significantly more feedback from other people $(M=14.25, S D=5.53)$ compared to sources other than people $(M=2.81, S D=5.59), p<.001, r=.81$. However, there were no significant differences in the percentage of overall feedback sought from other people ( $M=$ $14.25, S D=5.53)$ versus oneself $(M=12.52, S D=25.83), p>.05, r=.06$, or from oneself $(M=$ $12.52, S D=25.83)$ versus sources other than people $(M=2.81, S D=5.59), p>.05, r=.36$. In 
the GAD group there was a significant difference between these sources, $F(1.82,34.60)=4.44, p$ $<.05$, such that these individuals sought significantly more feedback from other people $(M=$ 15.69, $S D=3.53)$ compared to oneself $(M=4.80, S D=12.47), p<.01, r=.62$. However, there were no significant differences in the percentage of overall feedback sought from other people $(M=15.69, S D=3.53)$ versus sources other than people $(M=8.18, S D=14.77), p>.05, r=.41$, or from oneself $(M=4.80, S D=12.47)$ versus sources other than people $(M=8.18, S D=14.77)$, $p>.05, r=.18$. Similarly, in the healthy comparison group there was a significant difference between these sources, $F(1.47,28.01)=7.05, p<.01$, such that more feedback was sought from other people $(M=12.89, S D=8.20)$ compared to oneself $(M=1.39, S D=4.56), p<.001, r=$ .81. However, there were no significant differences in how often healthy individuals sought feedback from other people $(M=12.89, S D=8.20)$ versus sources other than people $(M=5.28$, $S D=13.80), p>.05, r=.42$, or oneself $(M=1.39, S D=4.56)$ versus sources other than people $(M=5.28, S D=13.80), p>.05, r=.26$. Refer to Table 7 for a summary of mean percentages of sources of overall feedback seeking over a 2-week period (based on diary records) stratified by group.

Additional MANOVAs were conduced to evaluate group differences in the frequency with which feedback was sought from each source as measured by the PFSF-Q and the NFSF-Q. Using Roy's Largest Root, there was a significant effect of group on sources of PFS, $\theta=.59$, $F(8,49)=3.61, p<.01$ (Wilk's Lambda and Hotelling's Trace were also significant, $p s<.05$, and Pillai's Trace approached significance, $p=.06$ ), with follow-up ANOVAs indicating significant group differences in the tendency to seek positive feedback from friends, $F(2,55)=$ $5.49, p<.01$, acquaintances, $F(2,55)=3.83, p<.05$, oneself, $F(2,55)=3.78, p<.05$, and sources other than people, $F(2,55)=5.55, p<.01$. Bonferroni tests revealed that individuals 
with GAD sought significantly more positive feedback than healthy individuals from friends ( $M$ $=3.05, S D=.85$ for GAD; $M=1.84, S D=1.34$ for healthy), $p<.01, r=.44$, acquaintances $(M=$ 1.47, $S D=1.17$ for GAD; $M=.68, S D=.75$ for healthy), $p<.05, r=.40$, and oneself $(M=2.84$, $S D=1.07$ for GAD; $M=1.84, S D=1.01$ for healthy), $p<.05, r=.40$. Individuals with SAD did not significantly differ from the other two groups in how often positive feedback was reportedly sought from friends $(M=2.55, S D=1.15$ for $\mathrm{SAD})$, acquaintances $(M=.85, S D=.81$ for $\mathrm{SAD})$, or oneself $(M=2.70, S D=1.49$ for $\mathrm{SAD}), p s>.05$. However, individuals with $\mathrm{SAD}(M=2.50$, $S D=1.43)$ and individuals with $\operatorname{GAD}(M=2.74, S D=1.37)$ reported seeking significantly more positive feedback from sources other than people compared to healthy individuals $(M=1.37, S D$ $=1.26), p<.05, r=.41$ and $p<.01, r=.50$, respectively, whereas there was no significant difference between the two clinical groups, $p>.05, r=.10$.

A series of multiple linear regressions were conducted to evaluate whether IUS, DASSD, BFNE, FPES, and STICSA scores significantly moderated the association between group and percentage of positive feedback sought from sources other than people. The only significant interaction was between the first dummy variable (individuals with SAD versus healthy individuals) and IUS scores (but not DASS-D or STICSA scores) in predicting the amount of positive feedback sought from others, $\beta=-.36, t(55)=-2.45, p<.05$. Simple effects analyses indicated that individuals with SAD sought significantly more feedback from sources other than people compared to healthy individuals at low levels of IU (i.e., scores one standard deviation below the mean), $t(57)=2.16, p<.05$, whereas there was no significant group difference at high levels of IU (i.e., scores one standard deviation above the mean), $t(57)=-1.80, p>.05$. Additional simple effects analyses indicated that healthy individuals sought significantly more feedback from sources other than people at high levels of IU compared to low levels, $t(57)=$ 
$5.33, p<.001$, whereas there was no significant association between feedback sought from sources other than people and levels of IU in individuals with SAD, $t(57)=-1.25, p>.05$. This interaction is displayed in Figure 2.

Lastly, there was no significant effect of group on sources of NFS, $V=.34, F(16,98)=$ 1.26, $p>.05$ (Wilk's Lambda, Hotelling's Trace, and Roy's Largest Root were also nonsignificant, $p s<.05)$.

\section{Hypothesis 4: There will be no significant differences between the two clinical} groups in feedback seeking triggered by social/evaluative concerns, whereas high worriers will seek significantly more feedback triggered by general threats. Triggers of feedback seeking were expected to fall under the category of social/evaluative threat or general threat. The author coded each diary record according to definitions of social/evaluative and general threats used in previous research (e.g., Cougle et al., 2012). Feedback seeking triggered by social/evaluative threats was defined as feedback "carried out so that the individual is assured that others do not think negatively of him or her" (Cougle et al., 2012, p. 118), and feedback seeking triggered by general threats was defined as feedback "carried out to receive assurance from others that negative outcomes will not occur" (Cougle et al., 2012, p. 118). Triggers that were neither social/evaluative nor general were coded as "other."

A series of MANOVAs were conducted to determine group effects on triggers of PFS, NFS, and overall feedback seeking. There were no significant group differences in PFS triggers, $V=.11, F(6,114)=1.11, p>.05, \mathrm{NFS}$ triggers, $V=.10, F(6,114)=1.00, p>.05$, or triggers of overall feedback seeking, $V=.09, F(6,114)=.93, p>.05$. Table 8 presents the mean percentages of triggers of overall feedback seeking over a 2-week period (based on diary records) stratified by group. 
Hypothesis 5: There will be no significant differences between the two clinical groups in PFS aimed at reducing social/evaluative concerns, whereas high worriers will engage in significantly more PFS aimed at reducing general threats. The author coded each diary record for functions of PFS according to the definitions of social/evaluative and general threats described above. A series of MANOVAs indicated that there were no significant group differences in the functions of PFS, $V=.15, F(6,114)=1.55, p>.05$. Table 9 presents the mean percentages of functions of PFS over a 2-week period (based on diary records) stratified by group.

Hypothesis 6: There will no significant differences between the two clinical groups in NFS designed to confirm negative self-views, whereas socially anxious individuals will engage in significantly more NFS designed to avoid appearing "too good" and to lower others' expectations compared to the other two groups. The author coded each diary record for the proposed functions of NFS. A series of MANOVAs indicated that there were no significant group differences in the functions of NFS, $V=.12, F(6,114)=1.20, p>.05$. Table 9 presents the mean percentages of functions of NFS over a 2-week period (based on diary records) stratified by group.

Hypothesis 7: There will be no significant differences between the two clinical groups in terminating PFS following reductions in social/evaluative concerns, whereas high worriers will be significantly more likely to terminate PFS following reductions in general threats. The author coded each diary record for termination of PFS according to the definitions of social/evaluative and general threats described above. A series of MANOVAs indicated that there were no significant group differences PFS termination criteria, $V=.17, F(6,114)=1.75, p$ 
$>.05$. Table 10 presents the mean percentages of PFS termination criteria over a 2-week period (based on diary records) stratified by group.

Hypothesis 8: There will be no significant differences between the two clinical groups in terminating NFS following the confirmation of negative self-views, whereas socially anxious individuals will be significantly more likely to terminate NFS following avoiding appearing "too good" and lower others' expectations compared to the other two groups. The author coded each diary record for termination of NFS according to the proposed termination criteria. A series of MANOVAs indicated that there were no significant group differences in NFS termination criteria, $V=.06, F(4,116)=.90, p>.05$. Table 10 presents the mean percentages of NFS termination criteria over a 2-week period (based on diary records) stratified by group.

Hypothesis 9: First, the receipt of positive feedback will lead to decreases in negative affective states and increases in positive affective states in individuals with GAD and healthy individuals, whereas the receipt of positive feedback will lead to increases in negative affective states and decreases in positive affective states in individuals with SAD. Second, the receipt of negative feedback will lead to increases in negative affective states and decreases in positive affective states within each group. Third, the receipt of either type of feedback (positive or negative) will lead to decreases in uncertainty and increases in certainty. Paired samples $t$-tests were conducted to evaluate changes in each negative affective state (i.e., fear, anxiety, sadness, frustration, disgust, anger, shame, guilt), each positive affective state (i.e., confidence, relaxation, happiness), uncertainty, and certainty following the receipt of positive and negative feedback during episodes of PFS and NFS within each group. 
In the healthy group, the receipt of positive feedback during episodes of PFS (i.e., when positive feedback was hoped for) was followed by nonsignificant decreases in fear, $t(15)=2.01$, $p>.05, r=.46$, sadness, $t(15)=1.42, p>.05, r=.34$, frustration, $t(15)=1.75, p>.05, r=.41$, shame, $t(15)=.30, p>.05, r=.08$, and guilt, $t(15)=.97, p>.05, r=.24$; nonsignificant increases in disgust, $t(15)=-1.36, p>.05, r=.33$, and anger, $t(15)=-1.20, p>.05, r=30$; and significant decreases in anxiety, $t(15)=2.57, p<.05, r=.55$, and uncertainty, $t(15)=4.78, p<$ $.001, r=.78$. The receipt of positive feedback during PFS was also followed by significant increases in confidence, $t(15)=-3.88, p>.01, r=.71$, relaxation, $t(15)=-3.37, p<.01, r=.66$, happiness, $t(15)=-4.48, p<.001, r=.76$, and certainty, $t(15)=-4.38, p<.01, r=.75$. See Table 11 for mean pre-, post-, and change scores for each affective state, certainty, and uncertainty after receiving positive feedback during episodes of PFS.

In the SAD group, the receipt of positive feedback during episodes of PFS was followed by significant decreases in fear, $t(17)=4.23, p<.01, r=.74$, anxiety, $t(17)=7.77, p<.001, r=$ .88 , sadness, $t(17)=4.90, p<.001, r=.77$, frustration, $t(17)=4.07, p<.01, r=.70$, anger, $t(17)$ $=2.62, p<.05, r=.54$, shame, $t(16)=2.56, p<.05, r=.54$, and uncertainty, $t(17)=9.42, p<$ $.001, r=.92$; and nonsignificant decreases in disgust, $t(17)=1.38, p>.05, r=.32$, and guilt, $t(17)=1.03, p>.05, r=.24$. Further, the receipt of positive feedback during PFS was followed by significant increases in confidence, $t(17)=-7.10, p>.001, r=.86$, relaxation, $t(17)=-6.67, p$ $<.001, r=.85$, happiness, $t(17)=-5.72, p<.001, r=.81$, and certainty, $t(17)=-7.55, p<.001, r$ $=.77$. See Table 11 for mean pre-, post-, and change scores for affective states, certainty, and uncertainty after receiving positive feedback during PFS.

In the GAD group, the receipt of positive feedback during episodes of PFS was followed by significant decreases in fear, $t(18)=4.37, p<.001, r=.72$, anxiety, $t(18)=5.77, p<.001, r=$ 
.81 , sadness, $t(18)=2.96, p<.01, r=.57$, frustration, $t(18)=4.15, p<.01, r=.70$, disgust, $t(18)$ $=2.40, p<.05, r=.49$, anger, $t(18)=3.28, p<.01, r=.61$, and uncertainty, $t(18)=6.74, p<$ $.001, r=.85$; and nonsignificant decreases in shame, $t(18)=1.97, p>.05, r=.42$, and guilt, $t(18)=.04, p>.05, r=.009$. In addition, the receipt of positive feedback during PFS was followed by significant increases in confidence, $t(18)=-4.02, p<.01, r=.69$, relaxation, $t(18)=$ $-4.51, p<.001, r=.73$, happiness, $t(18)=-3.97, p<.01, r=.68$, and certainty, $t(18)=-5.09, p<$ $.001, r=.77$. See Table 11 for mean pre-, post-, and change scores for each affective state, certainty, and uncertainty after receiving positive feedback during PFS.

In the healthy group, the receipt of negative feedback during PFS was followed by nonsignificant increases in fear, $t(4)=-.63, p>.05, r=.30$, anxiety, $t(4)=-.57, p>.05, r=.27$, sadness, $t(4)=-2.01, p>.05, r=.71$, frustration, $t(4)=-.53, p>.05, r=.26$, disgust, $t(4)=-$ $1.63, p>.05, r=.63$, anger, $t(4)=-1.51, p>.05, r=.60$, shame, $t(4)=-1.37, p>.05, r=.57$, and guilt, $t(4)=-.92, p>.05, r=.42$; a nonsignificant decrease in uncertainty, $t(4)=.34, p>.05$, $r=.17$; and nonsignificant decreases in confidence, $t(4)=1.25, p>.05, r=.53$, relaxation, $t(4)=$ 2.29, $p>.05, r=.75$, happiness, $t(4)=1.27, p>.05, r=.54$, and certainty, $t(4)=.16, p>.05, r=$ .09. See Table 12 for mean pre-, post-, and change scores for each affective state, certainty, and uncertainty after receiving negative feedback during PFS.

In the SAD group, the receipt of negative feedback during PFS was followed by nonsignificant increases in fear, $t(8)=-.74, p>.05, r=.25$, sadness, $t(8)=-1.64, p>.05, r=.50$, uncertainty, $t(8)=-.65, p>.05, r=.22$, frustration, $t(8)=-.43, p>.05, r=.15$, disgust, $t(8)=-$ $1.28, p>.05, r=.41$, anger, $t(8)=-.54, p>.05, r=.19$, and guilt, $t(8)=-.74, p>.05, r=.25 ; \mathrm{a}$ significant increase in shame, $t(8)=-3.18, p<.05, r=.75$; and a nonsignificant decrease in anxiety, $t(8)=-1.43, p>.05, r=.45$. The receipt of negative feedback during PFS was also 
followed by nonsignificant decreases in confidence, $t(8)=1.41, p>.05, r=.45$, happiness, $t(8)=$ $1.63, p>.05, r=.50$, and certainty, $t(8)=.12, p>.05, r=.04$; and a nonsignificant increase in relaxation, $t(8)=-.25, p>.05, r=.09$. See Table 12 for mean pre-, post-, and change scores for each affective state, certainty, and uncertainty after receiving negative feedback during PFS.

In the GAD group, the receipt of negative feedback during PFS was followed by nonsignificant increases in fear, $t(9)=-1.08, p>.05, r=.34$, anxiety, $t(9)=-.05, p>.05, r=.02$, sadness, $t(9)=-.77, p>.05, r=.25$, frustration, $t(9)=-.31, p>.05, r=.10$, disgust, $t(9)=-1.07$, $p>.05, r=.35$, anger, $t(9)=-1.57, p>.05, r=.46$, shame, $t(9)=-2.00, p>.05, r=.56$, and guilt, $t(9)=-1.28, p>.05, r=.39$; a nonsignificant decrease in uncertainty, $t(9)=.23, p>.05, r$ $=.07$; a nonsignificant decrease in confidence, $t(9)=1.48, p>.05, r=.44$, relaxation, $t(9)=.04$, $p>.05, r=.01$, and happiness, $t(9)=1.64, p>.05, r=.48$; and a nonsignificant increase in certainty, $t(9)=-.57, p>.05, r=.19$. See Table 12 for mean pre-, post-, and change scores for each affective state, certainty, and uncertainty after receiving negative feedback during PFS.

In the healthy group, the receipt of positive feedback during episodes of NFS (i.e., when negative feedback was hoped for) was followed by nonsignificant decreases in fear, $t(3)=1.20, p$ $>.05, r=.57$, sadness, $t(3)=1.86, p>.05, r=.73$, frustration, $t(3)=2.94, p>.05, r=.86$, disgust, $t(3)=1.00, p>.05, r=.50$, anger, $t(3)=1.71, p>.05, r=.70$, shame, $t(3)=2.91, p>$ $.05, r=.86$, and uncertainty, $t(3)=2.62, p>.05, r=.57$; significant decreases in anxiety, $t(3)=$ $3.88, p<.05, r=.83$, and guilt, $t(3)=4.06, p<.05, r=.92$; significant increases in confidence, $t(3)=-3.80, p<.05, r=.91$, happiness, $t(3)=-5.72, p<.05, r=.96$, and certainty, $t(3)=-4.83, p$ $<.05, r=.94$, and a nonsignificant increase in relaxation, $t(3)=-2.78, p>.05, r=.85$. See Table 13 for mean pre-, post-, and change scores for each affective state, certainty, and uncertainty after receiving positive feedback during NFS. 
In the SAD group, the receipt of positive feedback during NFS was followed by nonsignificant decreases in fear, $t(2)=1.51, p>.05, r=.73$, anxiety, $t(2)=1.61, p>.05, r=.75$, frustration, $t(2)=2.22, p>.05, r=.84$, disgust, $t(2)=1.00, p>.05, r=.58$, shame, $t(2)=1.89, p$ $>.05, r=.80$, uncertainty, $t(2)=.38, p>.05, r=.26$; no change in anger, $t(2)=.00, p>.05, r=$ .00 ; and nonsignificant increases in sadness, $t(2)=-.26, p>.05, r=.18$, and guilt, $t(2)=-1.00, p$ $>.05, r=.58$. Further, the receipt of positive feedback during NFS was followed by nonsignificant increases in confidence, $t(2)=-1.86, p>.05, r=.80$, happiness, $t(2)=-1.00, p>$ $.05, r=.58$, and certainty, $t(2)=-1.19, p>.05, r=.64$; and a nonsignificant decrease in relaxation, $t(2)=.50, p>.05, r=.33$. See Table 13 for mean pre-, post-, and change scores for each affective state, certainty, and uncertainty after receiving positive feedback during NFS.

In the GAD group, the receipt of positive feedback during NFS was followed by nonsignificant decreases in fear, $t(4)=2.30, p>.05, r=.75$, anxiety, $t(4)=1.05, p>.05, r=.46$, sadness, $t(4)=1.61, p>.05, r=.63$, frustration, $t(4)=1.61, p>.05, r=.63$, disgust, $t(4)=1.25$, $p>.05, r=.53$, anger, $t(4)=2.26, p>.05, r=.75$, shame, $t(4)=1.15, p>.05, r=.50$, guilt, $t(4)$ $=.30, p>.05, r=.15$; a significant decrease in uncertainty, $t(4)=3.70, p<.05, r=.88$; and nonsignificant increases in confidence, $t(4)=-1.30, p>.05, r=.54$, relaxation, $t(4)=-1.61, p>$ $.05, r=.63$, happiness, $t(4)=-2.14, p>.05, r=.73$, and certainty, $t(4)=-1.63, p>.05, r=.63$ See Table 13 for mean pre-, post-, and change scores for each affective state, certainty, and uncertainty after receiving positive feedback during NFS.

In the healthy group, the receipt of negative feedback during NFS was followed by nonsignificant decreases in fear, $t(5)=1.17, p>.05, r=.46$, anxiety, $t(5)=2.03, p>.05, r=.67$, sadness, $t(5)=.66, p>.05, r=.28$, frustration, $t(5)=1.02, p>.05, r=.41$, and uncertainty, $t(5)$ $=.40, p>.05, r=.18$; and nonsignificant increases in disgust, $t(5)=-1.18, p>.05, r=.25$, 
anger, $t(5)=-.98, p>.05, r=.40$, shame, $t(5)=-.30, p>.05, r=.13$, guilt, $t(5)=-.75, p>.05, r$ $=.32$. The receipt of negative feedback during NFS was also followed by nonsignificant decreases in confidence, $t(5)=1.46, p>.05, r=.55$, and nonsignificant increases in relaxation, $t(5)=-.57, p>.05, r=.25$, happiness, $t(5)=-.86, p>.05, r=.36$, and uncertainty, $t(5)=-.89, p$ $>.05, r=.37$. See Table 14 for mean pre-, post-, and change scores for each affective state, certainty, and uncertainty after receiving negative feedback during NFS.

In the SAD group, the receipt of negative feedback during NFS was followed by nonsignificant decreases in fear, $t(4)=.45, p>.05, r=.22$, anxiety, $t(4)=1.63, p>.05, r=.63$, uncertainty, $t(4)=.14, p>.05, r=.07$, anger, $t(4)=.30, p>.05, r=.15$, shame, $t(4)=.39, p>$ $.05, r=.19$, and guilt, $t(4)=1.50, p>.05, r=.60$; no change in frustration, $t(4)=.00, p>.05, r$ $=.00$; and nonsignificant increases in sadness, $t(4)=-.70, p>.05, r=.33$, disgust, $t(4)=-1.50, p$ $>.05, r=.60$, confidence, $t(4)=-.22, p>.05, r=.11$, relaxation, $t(4)=-.75, p>.05, r=.35$, happiness, $t(4)=-.49, p>.05, r=.30$, and certainty, $t(4)=-1.41, p>.05, r=.58$, See Table 14 for mean pre-, post-, and change scores for each affective state, certainty, and uncertainty after receiving negative feedback during NFS.

In the GAD group, the receipt of negative feedback during NFS was followed by nonsignificant decreases in fear, $t(7)=.56, p>.05, r=.21$, anxiety, $t(7)=1.76, p>.05, r=.55$, frustration, $t(7)=.68, p>.05, r=.25$, anger, $t(7)=.66, p>.05, r=.24$, shame, $t(7)=.15, p>$ $.05, r=.05$; nonsignificant increases in sadness, $t(7)=-1.27, p>.05, r=.43$, and disgust, $t(7)=$ $.07, p>.05, r=.03$; a significant increase in guilt, $t(7)=-2.77, p<.05, r=.73$; and no change in uncertainty, $t(7)=.00, p>.05, r=.00$. The receipt of feedback during NFS was also followed by nonsignificant increases in confidence, $t(7)=-.33, p>.05, r=.12$, relaxation, $t(7)=-.92, p>$ $.05, r=.33$, and certainty, $t(7)=-1.46, p>.05, r=.48$; and a nonsignificant decrease in 
happiness, $t(7)=.30, p>.05, r=.11$. See Table 14 for mean pre-, post-, and change scores for each affective state, certainty, and uncertainty after receiving negative feedback during NFS.

Exploratory Question: Are there significant group differences in how much negative affective states, positive affective states, uncertainty, and certainty change following the receipt of positive and negative feedback during episodes of PFS and NFS? A series of oneway ANOVAs were conducted to evaluate group differences in the degree to which negative affective states (i.e., fear, anxiety, sadness, frustration, disgust, anger, shame, guilt), positive affective states (i.e., confidence, relaxation, happiness), uncertainty, and certainty changed following the receipt of positive and negative feedback during PFS and NFS within each group.

Following the receipt of positive feedback during episodes of PFS, there was a significant group difference in the degree to which anxiety decreased, $F(2,50)=5.64, p<.01$, such that individuals with SAD experienced a significantly greater reduction in anxiety than healthy individuals. There was a significant group difference in how much sadness decreased, $F(2,50)=$ $4.23, p<.05$, such that individuals with SAD experienced a significantly greater reduction in sadness than healthy individuals. There was a significant group difference in how much anger decreased, $F(2,50)=5.11, p<.05$, such that individuals with SAD and individuals with GAD both experienced a significantly greater reduction in anger than healthy individuals (in fact, healthy individuals reported a nonsignificant increase in anger). There was a significant group difference in changes in certainty, $F(2,50)=3.36, p<.05$, such that individuals with SAD experienced a significantly greater increase in certainty than individuals with GAD. There were no significant group differences in the degree to which affective states, uncertainty, or certainty changed following the receipt of negative feedback during PFS. See Table 11 and Table 12 for 
mean pre-, post-, and change scores for each affective state, certainty, and uncertainty after receiving positive feedback and negative feedback during PFS stratified by group, respectively. Following the receipt of positive feedback during episodes of NFS, the only significant group difference was in the extent to which guilt decreased, $F(2,9)=5.28, p<.05$, such that healthy individuals experienced a significantly greater reduction in guilt compared to individuals with SAD (in fact, individuals with SAD experienced a nonsignificant increase in guilt). There were no significant group differences in the degree to which affective states, uncertainty, or certainty changed following the receipt of negative feedback during NFS. See Table 13 and Table 14 for mean pre-, post-, and change scores for each affective state, certainty, and uncertainty after receiving positive feedback and negative feedback during NFS stratified by group, respectively. 


\section{Discussion}

The purpose of the current study was to investigate various features of PFS and NFS in individuals with SAD compared to individuals with GAD and individuals with no lifetime history of mental health difficulties to determine whether certain forms and features of feedback seeking were specific to SAD. The prediction that individuals with SAD and individuals with GAD would engage in significantly more PFS, NFS, and overall feedback seeking (PFS and NFS combined) than healthy individuals as determined by the number of completed FSDs was not supported. However, when the frequency of overall feedback seeking was adjusted for participants' reported compliance ratings, individuals with GAD were found to engage in significantly more overall feedback seeking than healthy individuals. Further, increases in IUS scores significantly predicted increases in the frequency of overall feedback seeking, but only for individuals with GAD. Importantly, the lack of significant group differences in the frequency of PFS, NFS, and overall feedback seeking (not adjusted for compliance) does not reflect similarly high levels of these behaviours across groups, but rather similarly low levels of these behaviours, which was unexpected given that these behaviours are consistently described as "excessive" or problematic in the literature. In the SAD group, the mean frequency was 5.67 for PFS and .90 for NFS; in the GAD group, the the mean frequency was 7.90 for PFS and 1.35 for NFS; and in the healthy group, the mean frequency was 5.85 for PFS and 1.05 for NFS. During the 2-week period, all three groups reported engaging in significantly more PFS than NFS.

Consistent with predictions, there were no significant differences between individuals with SAD and individuals with GAD in their tendency to seek positive, negative, or overall feedback about social/evaluative concerns according to the FSDs. Contrary to predictions, there were no significant differences between these two groups in their tendency to seek positive, 
negative, or overall feedback about general safety/security concerns. Although no specific predictions were made about differences between the two clinical groups and healthy individuals on topics of feedback seeking, the PFSF-Q and NFSF-Q, which were designed to provide a traitlike measure of PFS and NFS, respectively, indicated that individuals with SAD and individuals with GAD (combined) sought significantly more feedback about social/evaluative, general, and "other" concerns compared to healthy individuals, whereas no significant differences were found between the two clinical groups, which corroborated findings based on the FSDs.

The prediction that individuals with GAD would seek feedback from a significantly greater number of sources than individuals with SAD and healthy individuals, and that individuals with SAD would seek feedback from a significantly greater number of sources than healthy individuals (as measured by the FSDs) was not supported. Further, according to the FSDs, there were no significant group differences in the percentage of feedback sought from each individual source. However, within each group, there were significant differences in how often feedback was sought from other people (e.g., romantic partner, friend), oneself, and sources other than people (e.g., websites, books), with other people being the most common source of feedback for all three groups. According to the PFSF-Q, the two clinical groups sought significantly more feedback from sources other than people than healthy individuals. However, further analyses revealed that individuals with SAD sought significantly more positive feedback from sources other than people compared to healthy individuals only at low levels of IU.

Hypotheses regarding group differences in the triggers, functions, and termination criteria of feedback seeking were only partially supported. As predicted, there were no significant differences between individuals with SAD and individuals with GAD in their tendency to seek positive or negative feedback triggered by social/evaluative concerns. In fact, none of the groups 
differed significantly in terms of social/evaluative triggers. Contrary to predictions, there were no significant group differences in PFS or NFS triggered by general concerns. Also, as predicted, individuals with SAD and individuals with GAD did not differ significantly in their tendency to engage in PFS aimed at reducing social/evaluative concerns; however, once again none of the groups significantly differed in this respect. Inconsistent with predictions was the finding that the two clinical groups did not differ significantly in their tendency to seek positive feedback for the purpose of reducing general concerns. Similarly, as expected, there were no significant differences between the two clinical groups in their tendency to terminate PFS due to reductions in social/evaluative concerns. Contrary to predictions, individuals with GAD were not more likely than individuals with SAD to terminate PFS following reductions in general concerns.

As expected, there were no significant differences between the two clinical groups in their tendency to engage in NFS aimed at confirming negative self-views. In fact, none of the groups differed in regards to this proposed function of NFS. Further, the prediction that individuals with SAD would engage in significantly more NFS in order to avoid appearing "too good" or to lower others' standards of them compared to the other two groups was not supported. The prediction that the two clinical groups would not differ significantly in their tendency to terminate NFS due to the confirmation of self-beliefs was supported, whereas the prediction that individuals with SAD would be more likely to terminate NFS following reductions in appearing "too good" and lowering others' expectations of them was not supported.

Consistent with hypotheses, the receipt of positive feedback during PFS was generally followed by decreases in negative affective states and uncertainty and increases in positive affective states and certainty in all three groups. However, changes in negative affective states in the healthy group were largely nonsignificant with the exception of anxiety and uncertainty, 
which was likely an artifact of very low levels of these negative affective states to begin with (i.e., prior to seeking feedback). The receipt of negative feedback during PFS was generally followed by increases in negative affective states, and decreases in positive affective states, albeit not to a significant degree for all affective states, and nonsignificant changes in uncertainty and certainty in all three groups. Further, the number of participants who engaged in NFS and ended up receiving positive or negative feedback was very small; three participants in the SAD group, five participants in the GAD group, and four participants in the healthy group engaged in NFS and received positive feedback, whereas five participants in the SAD group, eight participants in the GAD group, and six participants in the healthy group engaged in NFS and received negative feedback, thereby limiting the interpretability of findings regarding how much affective states, uncertainty, and certainty changed in each group during NFS.

In terms of group differences, individuals with $\mathrm{SAD}$ reported a significantly greater reduction in anxiety and sadness than healthy individuals, whereas both clinical groups reported a significantly greater reduction in anger than healthy individuals following the receipt of positive feedback during PFS. Individuals with SAD also reported a significantly greater increase in certainty than individuals with GAD following the receipt of positive feedback during PFS. Further, following the receipt of positive feedback during NFS, healthy individuals experienced a greater reduction in guilt compared to individuals with SAD (in fact, individuals with SAD experienced a nonsignificant increase in guilt).

The finding that there were no significant group differences in the frequency of PFS based on the FSDs is inconsistent with previous research demonstrating higher levels of reassurance seeking in socially anxious individuals (Heerey \& Kring, 2007) and in individuals with a diagnosis of GAD (Beesdo-Baum et al., 2012) compared to nonsocially anxious 
individuals and healthy controls, respectively. The finding that there were no significant group differences in the frequency of NFS is also unexpected given recent evidence suggesting that socially anxious individuals preferentially seek negative feedback from others (Valentiner et al., 2011) - a behaviour that is believed to manifest more frequently in individuals with negative self-beliefs as an attempt to confirm these beliefs (North \& Swann, 2009) in order to maintain a coherent sense of self and establish greater control and predictability in their social worlds (e.g., to ensure that others have realistic expectations of them) (see Evraire \& Dozois, 2011). There are a few possible explanations for these null findings, including the fact that previous studies (e.g., Heerey \& Kring, 2007; Valentiner et al., 2011) used analogue samples of undergraduate students, thereby limiting the generalizability of previous research findings to clinical samples, which were used in the current study. Whereas individuals with elevated, but nonclinical levels of social anxiety may frequently solicit feedback from others in order to feel more secure and certain about the status of their interpersonal interactions and relationships (e.g., Heerey \& Kring, 2007), individuals with a diagnosis of SAD may fear the probability of behaving in a socially inappropriate manner and the perceived consequences for doing so (e.g., reduced social standing, rejection) (Clark \& Wells, 1995) to such an extent that they avoid engaging in certain interpersonal behaviours that could lead to negative evaluation, including feedback seeking. Thus, there may be a dissociation in the frequency of feedback seeking between individuals with moderate, subclinical levels of social anxiety and individuals with severe SAD, which represents an intriguing topic for future research.

An alternative explanation for these null findings is the methodology used in the current study, which was a daily diary method that used event-contingent recording (Moskowitz \& Young, 2006), in which participants were asked to complete a diary record every time they 
engaged in a feedback seeking behaviour over 2 weeks. Although the daily diary method has several advantages over more traditional methods of assessing behaviours (e.g., self-report questionnaires), including greater ecological validity and reduced recall bias, this method has a number of potential drawbacks (Gunthert \& Wenze, 2012). A phenomenon called measurement reactivity, which "occurs when the act of repeated assessment systematically changes participants' behaviours or responses" (Gunthert \& Wenze, 2012, p. 147) can compromise the validity of daily diary studies. For example, it is possible that asking participants to monitor and record their feedback seeking behaviours, which may have fostered greater self-awareness, caused them to systematically change their behaviours. As mentioned earlier, one FSD was removed because the participant stated that he or she considered seeking negative feedback, but ultimately decided not to engage in this behaviour. This participant, who was in the GAD group, reported that not acting on this urge was likely a result of participating in the study, and that under normal circumstances he or she would have sought feedback. This recorded instance of failing to act on an urge that would have otherwise manifested in the form of feedback seeking is a clear example of measurement reactivity, raising questions about how often participants experienced such urges, but chose not to act on them, whereas under more realistic circumstances (i.e., when not participating in a study that involved monitoring and recording their behaviours) they would have readily sought feedback.

Measurement reactivity may have masked significant group differences in the frequency of PFS and NFS if differences did, in fact, exist. This phenomenon may have been particularly problematic for individuals with $\mathrm{SAD}$ if during the monitoring and recording process these individuals suspected that feedback seeking was a socially aversive behaviour that could potentially lead to negative evaluation by others in naturalistic contexts, which is consistent with 
socially anxious individuals' tendency to hold negative self-images (e.g., images of the self behaving in a socially inept manner) and to overestimate the negative consequences of social behaviours, which may have led to intentional avoidance of feedback seeking (Clark \& Wells, 1995; see Hoffman, 2007 for a review). Concerns around social desirability and self-presentation may have further contributed to an underreporting of feedback seeking behaviours in individuals with SAD (Schwarz, 2012). That is, individuals with SAD may have been concerned that the experimenter of the current study would evaluate them unfavourably for reporting repeated feedback seeking episodes over the 2-week period (as indicated by the number of completed FSDs). Unfortunately, the current study did not measure individuals' urges to seek feedback versus actually engaging in feedback seeking, which represents a limitation of this study and an important area for future research. In addition, motivational and personality factors may have biased participants' daily diary recording, such that individuals with lower levels of motivation and conscientiousness may have been less compliant with daily monitoring and recording. Of note, individuals with SAD and individuals with GAD had significantly higher depressive scores than healthy individuals, which may have contributed to decreased motivation and thus lower levels of compliance (both clinical groups had lower compliance ratings than healthy individuals, albeit not to a significant degree). Indeed, participants' depression scores were significantly negatively correlated with their reported compliance ratings, $r=-.33, p<.05$, such that higher levels of depression were associated with a lower tendency to capture each episode of feedback seeking in the FSD.

As previously mentioned, in addition to the absence of significant group differences in the frequency of PFS, NFS, and overall feedback seeking (not adjusted for compliance ratings), the frequency of feedback seeking behaviours within each group was quite low. Table 2 
demonstrates that the mean frequency of overall feedback seeking (adjusted for compliance) was approximately 9 times for individuals with SAD, 13 times for individuals with GAD, and 8 times for healthy individuals over a 2-week period, raising the question of whether feedback seeking in the current sample can be considered pathological or excessive in the first place. Some major limitations of the extant literature on feedback seeking behaviours in the context of anxiety disorders and depression (the majority of research to date has focused on feedback seeking in depression) is, first, the lack of clear definitions of feedback seeking behaviours and, second, a lack of consensus regarding when these behaviours are considered problematic or excessive, as there are currently no normative data on these behaviours (Evraire \& Dozois, 2011). For instance, in the Heerey and Kring (2007) study, a wide range of behaviours that were perceived as "soliciting support" from interaction partners were coded as reassurance seeking, including complaints (e.g., "I get nervous doing research studies"), apologies (e.g., "I apologize for interrupting"), and direct requests for feedback (e.g., "What should I do?"), which may explain the higher frequency with which participants were reported to engage in this behaviour compared to that observed in the current study. Based on this broad definition of reassurance seeking, socially anxious individuals in the Heerey and Kring study sought reassurance, on average, 4.64 times, and nonsocially anxious individuals sought reassurance, on average, 2.72 times during a 5-minute interaction of "getting to know one another." Participants in the current study sought positive, reassuring feedback at a substantially lower rate, which may be due, at least in part, to the narrower definition of PFS used in this study (i.e., "seeking positive, reassuring self-relevant information from different sources often in the form of direct verbal requests"). Further, BeesdoBaum and colleagues (2012) assessed reassurance seeking using just one item (i.e., "sought reassurance from other people") that participants rated on a 9-point scale (ranging from $0=N o t$ 
at all to $4=$ Moderate to $8=$ All the time) based on how frequently they had engaged in reassurance seeking over the past week, which yielded even lower rates of reassurance seeking than those in the current study, with healthy individuals scoring, on average, 1.18 and GAD patients scoring, on average, 2.71 (i.e., somewhere between Not at all and Moderate). Taken together, these findings suggest that the way in which feedback seeking behaviours are operationally defined can greatly influence the self-reported or observed frequency of these behaviours, which may explain the discrepant findings across studies of feedback seeking behaviours in anxious individuals.

As discussed in a recent review by Dozois and Evraire (2012), the absence of normative data on feedback seeking behaviours makes it difficult to determine when these behaviours can be deemed excessive. Does feedback seeking become excessive or problematic according to its frequency, its associated affective states (e.g., how much affective states change following feedback seeking), or its adverse interpersonal consequences (or a unique combination of these factors)? Although more research is required to adequately address the question of what differentiates adaptive or normative manifestations of feedback seeking from more pathological forms, researchers have suggested that there are "secure and nonsecure forms of ERS" (Evraire \& Dozois, 2011, p. 1295). Anxious individuals may engage in "nonsecure" forms of feedback seeking during which they express high levels of distress (e.g., fear, anxiety), which may elicit negative affect (e.g., frustration) in interaction partners and, in turn, lead to negative interpersonal outcomes, such as rejection by others (Coyne, 1976). Healthy individuals, on the other hand, may engage in more "secure" forms of feedback seeking during which they express lower levels of distress. This more adaptive form of feedback seeking may not be associated with the same adverse interpersonal consequences and may even facilitate healthy social interactions. 
Whether or not individuals with SAD or GAD express higher levels of distress during feedback seeking (e.g., through verbal or nonverbal cues, such as tone of voice) compared to healthy individuals, which may lead to differential psychological and interpersonal outcomes, such as negative evaluation or rejection by others, represents an interesting topic for future research.

The current study's findings of significant group differences in the extent to which affective states and certainty changed following the receipt of positive and negative feedback may shed some light on the question surrounding what makes feedback seeking problematic. The finding that individuals with SAD experienced a significantly greater decrease in levels of anxiety and sadness than healthy individuals after receiving positive feedback during episodes of PFS - a finding that is inconsistent with the BFOE model (Weeks \& Howell, 2012), which posits that individuals with SAD fear positive evaluation and are therefore expected to experience increases in negative affect in response to positive feedback - suggests that feedback seeking behaviours may be more strongly negatively reinforced in individuals with SAD compared to healthy individuals. To test whether feedback seeking is indeed a safety behaviour that maintains social anxiety over time, as it has been suggested in the literature (Helbig-Lang \& Petermann, 2010), a prospective study should be implemented to examine whether feedback seeking reduces negative affect in the short-term (as demonstrated in the current study), but increases or maintains negative affect in the long-term. Both clinical groups also experienced a significantly greater decrease in anger following the receipt of positive feedback during PFS compared to healthy individuals, again suggesting that feedback seeking may be more strongly negatively reinforced in the clinical groups due to greater short-term reductions in anger. Previous research has demonstrated elevated levels of anger in response to perceived negative evaluation and unfair treatment in socially anxious individuals (e.g., Erwin, Heimberg, Schneier, \& Liebowitz, 
2003), and heightened levels of several dimensions of anger (e.g., trait anger, hostility) in a GAD analogue sample (e.g., Deschênes, Dugas, Fracalanza, \& Koerner, 2012), suggesting that perhaps individuals with SAD and individuals with GAD sought positive feedback in order to curtail high levels of anger. However, as noted earlier, prospective studies are needed to examine whether feedback seeking maintains or worsens anger over time in individuals with SAD and individuals with GAD. Importantly, the two clinical groups may have experienced significantly greater reductions in certain negative affective states compared to healthy individuals simply because these states were higher in these two groups to begin with and therefore had more room to decrease than in the healthy individuals. In addition, the finding that healthy individuals experienced a reduction in guilt following the receipt of positive feedback during NFS, whereas individuals with SAD experienced no significant change in guilt indicates that the receipt of positive feedback alleviates guilt in healthy individuals, but has little impact on levels of guilt for individuals with SAD.

Although expected group differences did not emerge in the frequency of PFS, NFS, and overall feedback seeking for the potential reasons discussed above, when the frequency of overall feedback seeking (PFS and NFS combined) was adjusted for participants' self-reported compliance ratings (i.e., the percentage of feedback seeking behaviours that participants estimated they were able to capture in the diary records) there was a significant group difference, such that individuals with GAD engaged in significantly more overall feedback seeking than healthy individuals. This finding is consistent with previous research demonstrating higher levels of reassurance seeking in GAD patients compared to healthy individuals (e.g., Beesdo-Baum et al., 2012, Townsend et al., 1999). Further, this finding suggests that earlier null findings were due, at least in part, to participants' failure to accurately capture each instance of feedback 
seeking in the diary records. The overall mean compliance rating was $79 \%$, which despite being quite high was based on retrospective self-report and therefore response biases must be considered when interpreting these findings. Further analyses indicated that group (individuals with GAD versus healthy individuals) significantly moderated the association between IU and overall feedback seeking (adjusted for compliance), such that this association was significant only for individuals with GAD. Although the purpose of the current study was to evaluate whether specific forms or features of feedback seeking were specific to SAD, this finding is nonetheless important given its potential theoretical and therapeutic implications for GAD and therefore warrants further discussion.

The Intolerance of Uncertainty Model (IUM) of GAD (Dugas, Gagnon, Ladouceur, \& Freeston, 1998; Ladouceur et al., 1999) posits that holding beliefs that uncertainty is negative and threatening plays a crucial role in the development and maintenance of excessive worry. Individuals with GAD are proposed to have strong, negative responses to even small doses of uncertainty or ambiguity regardless of the probability of an uncertain event occurring, which has been described as an "allergic-type reaction" to uncertainty (Dugas \& Robichaud, 2007). In response to elevated levels of IU and subsequent worry and anxiety, individuals with GAD engage in a number of maladaptive behaviours (i.e., safety behaviours) that are designed to reduce or eliminate uncertainty, including reassurance seeking (see Dugas \& Robichaud, 2007). This low threshold for uncertainty may explain, at least in part, why individuals with SAD reported a significantly greater increase in certainty following the receipt of positive feedback during PFS compared to individuals with GAD; perhaps individuals with GAD required more instances of positive feedback or reassurance (and from multiple sources) to experience similar increases in levels of certainty as that observed in socially anxious individuals. 
The current study's finding of a moderating effect of group on the association between IU and overall feedback seeking (adjusted for compliance) begs the question as to why IU significantly predicted feedback seeking in individuals with GAD, but not in healthy individuals. One possible explanation for this finding is that individuals with GAD and healthy individuals use different strategies to cope with IU. Whereas individuals with GAD may feel compelled to excessively seek feedback from others when faced with uncertainty, healthy individuals may engage in alternative behaviours shown to be associated with IU, such as procrastinating, refusing to delegate tasks to others, or not fully committing to projects (Dugas \& Robichaud, 2007). Whether there are more or less adaptive ways to cope with uncertainty and whether these strategies differ between individuals with GAD and healthy individuals (or other diagnostic groups) represents an interesting direction for future research.

In addition, worry has been linked to a number of dysfunctional beliefs surrounding one's ability to solve problems, collectively known as a negative problem orientation (Robichaud \& Dugas, 2005). Individuals with this orientation tend to view problems as threatening, undermine their ability to solve problems, experience negative affect in response to problem solving, and anticipate a negative outcome regardless of their problem-solving efforts, which can maintain or worsen worry (see Koerner \& Dugas, 2006). Although negative problem orientation was not measured in the current study, previous research and cognitive theories of GAD would suggest that in response to uncertain situations that require some form of problem-solving, individuals with GAD might avoid or delay solving their problems, which is a behavioural consequence of a negative problem orientation (Dugas \& Robichaud, 2007). Instead of attempting to solve their problems, individuals with GAD may depend on others by excessively requesting reassurance (e.g., asking loved ones if everything is going to be alright). Healthy individuals, on the other 
hand, have been shown to have a less negative problem orientation than individuals with GAD (Dugas et al., 1998), suggesting that healthy individuals have more confidence in their problemsolving abilities and may therefore engage in more adaptive problem-solving behaviours (even in the context of elevated IU). Although there is some evidence of an association between IU and deficits in problem solving (Tallis \& Eysenck, 1994), this may hold only in the context of excessive worry. As such, it is possible that healthy individuals with increasing levels IU will not necessarily endorse a negative problem orientation, and may therefore engage in less feedback seeking as a means to cope with their problems as compared to individuals with GAD.

The finding that there were no significant group differences in the range or type of sources from which feedback was sought (based on the FSDs) is unexpected given the wide range of worry topics in GAD (Woody \& Rachman, 1994) and the significantly higher depressive scores in individuals with SAD and individuals with GAD compared to healthy individuals. This finding may be due, at least in part, to the methodology of the current study given that an average of $20 \%$ of participants' feedback seeking behaviours were reportedly not recorded. However, when sources were categorized into other people (e.g., romantic partner, friend), oneself, and sources other than people (e.g., websites), there were significant differences in the amount of overall feedback sought from each category. Within each group, the most common source of feedback were other individuals, which is consistent with research demonstrating that feedback seeking is a highly interpersonal process with potential interpersonal implications (Evraire \& Dozois, 2011; Heerey \& Kring, 2007). The finding that individuals with SAD were most likely to seek feedback from other individuals compared to oneself and sources other than people is particularly interesting in light of current cognitivebehavioural models of SAD suggesting that socially anxious individuals overestimate the 
occurrence of negative social outcomes, resulting in avoidance and safety behaviours (Hofmann, 2007). Perhaps in some social contexts, the need to gain assurance from others that one is not being negatively evaluated (via feedback seeking) competes with, and overrides the fear of interacting with others. It is also noteworthy that there were no significant differences in how often individuals with SAD sought feedback from other people versus oneself, which is consistent with cognitive-behavioural conceptualizations of SAD regarding heightened selffocused attention in the context of perceived social threat. The tendency to shift attention inward during social interactions may lead socially anxious individuals to seek feedback from themselves as they continually monitor their own behaviours and signs of anxiety.

Further, individuals with SAD reported seeking significantly more positive feedback from external sources (e.g., websites) compared to healthy individuals (based on the PFSF-Q), but only at lower levels of IU. At lower levels of IU, the mean rating of how often individuals with SAD sought feedback from external sources was 3, indicating "Often." This type of feedback seeking could be particularly problematic for individuals with SAD given the proliferation of social media sites that can easily generate social comparisons. Previous research demonstrated that individuals with SAD engaged in significantly more upward social comparisons, which involves comparing oneself to individuals perceived as being better off, and significantly fewer downward social comparisons, which involves comparing oneself to others perceived as lesser or worse off, compared to healthy individuals (Antony et al., 2005). The tendency to engage in upward social comparisons while seeking feedback on social media sites (e.g., Facebook, Instagram) or other external sources (e.g., fashion magazines) may contribute to negative self-views and exacerbate social anxiety. Interestingly, as IU increased, the frequency with which individuals with SAD sought feedback from external sources decreased, suggesting 
that at higher levels of IU individuals with SAD seek feedback from alternative sources, such as other individuals.

Contrary to predictions, there were no significant group differences in the topics, triggers, functions, or termination criteria of feedback seeking based on the FSDs, which is inconsistent with the cognitive and behavioural features of SAD (Clark \& Wells, 1995) and GAD (Woody \& Rachman, 1994). There are several possible explanations for these null findings. First, the three groups may, in fact, not significantly differ on these features of feedback seeking. That is, the feedback seeking behaviours of individuals with SAD, individuals with GAD, and healthy individuals may focus on similar topics, have similar triggers, serve similar functions, and be terminated for similar reasons. However, as demonstrated in Table 6, group differences on certain topics of feedback seeking, including signs of anxiety and safety of others, approached significance, such that individuals with SAD sought a greater percentage of feedback about these topics compared to the other two groups. The finding that individuals with SAD sought more feedback about signs of anxiety is not surprising given that exhibiting signs of anxiety (e.g., sweating, shaking, stuttering) is a core diagnostic feature of SAD. The finding that individuals with SAD sought more feedback about others' safety, however, is unexpected given that concerns about the safety and wellbeing of others is considered to be more characteristic of GAD than SAD. The percentage of feedback seeking about other topics, such as personal abilities, decision-making, and level of importance, were very similar across all three groups.

Further, individuals with SAD, individuals with GAD, and healthy individuals may significantly differ on triggers, functions, and termination criteria of feedback seeking that were not explicitly addressed and coded in the current study (e.g., boredom may trigger some instances of PFS and desire for improvement may trigger some instances of NFS) - a topic that 
warrants further investigation. An alternative explanation for these null findings is the methodology of the current study. Since participants, on average, did not record approximately $20 \%$ of their feedback seeking behaviours it is possible that important data regarding specific features of their behaviours were missed and therefore true significant differences, if any, were not found. Another possible explanation for the null findings regarding group differences in the frequency and various features of feedback seeking may be that the two clinical groups were not as distinct as expected. For example, individuals with SAD and individuals with GAD did not significantly differ on self-report measures of worry, IU, FNE, and FPE. Further, nearly half of the SAD group scored above the clinical cutoff score on the GAD-Q-IV and the PSWQ, whereas $85 \%$ of the GAD group scored above the clinical cutoff score on the SPIN, raising concerns about diagnostic overlap between the two clinical groups.

Further, the sample in the current study may have been too small to detect any significant differences. As such, future research should examine differential group effects on these various features of feedback seeking in a larger sample, and perhaps use an ecological momentary assessment method, in which participants are prompted several times per day (rather than one daily email reminder) ideally on some type of mobile device to further increase levels of compliance and reduce response bias (Gunthert \& Wenze, 2012). Future research should also implement an experimental design to evaluate potential group differences in feedback seeking in order to increase internal validity and establish causal relations of feedback seeking to anxiety and worry.

Despite these limitations, the current study provides preliminary evidence that what determines whether feedback seeking is excessive may not be based on frequency, but rather on other features of feedback seeking, such as changes in affective states following the receipt of 
feedback or the sources of feedback seeking, which has been suggested in previous research (see Evraire \& Dozois, 2011). In terms of the aspects of feedback seeking that are specific to individuals with SAD, the current study found that individuals with SAD experienced significantly greater reductions in anxiety, sadness, and anger than healthy individuals, and a significantly greater increase in certainty than individuals with GAD following the receipt of positive feedback during PFS. As noted earlier, future research should aim to extend the current findings by examining whether feedback seeking behaviours in SAD prospectively predict increases in negative affective states over time. This type of research could have important implications for advancing the cognitive model of SAD by incorporating feedback seeking as another safety behaviour that reduces negative affective states in the short-term (as demonstrated in the current study), but contributes to the maintenance or worsening of social anxiety in the long-term, which could have clinical implications for targeting this behaviour in treatment.

Further, individuals with SAD were significantly more likely than healthy individuals to seek feedback from external sources (e.g., websites, books) (albeit only at low levels of IU). Given research demonstrating the tendency for individuals with SAD to engage in upward social comparisons, leading to increases in social anxiety (Antony et al., 2005), it may be important to assess the sources from which patients seek feedback if this behaviour is deemed problematic. Further, although the purpose of the current study was to evaluate unique aspects of feedback seeking to SAD, the finding that that IU significantly predicted overall feedback seeking (adjusted for compliance) in individuals with GAD, but not in healthy individuals is nonetheless an intriguing finding with potentially important clinical implications for targeting feedback seeking in treatment. Indeed, existing treatment manuals for GAD propose that reassurance seeking is a behavioural manifestation of IU and thus IU is the primary treatment target for 
reducing maladaptive behaviours that contribute to worry (Dugas \& Robichaud, 2007). Taken together, the current study provides insight into the feedback seeking behaviours of individuals with SAD, individuals with GAD, and healthy individuals with findings suggesting that certain features of feedback seeking (e.g., changes in affective states, sources) are specific to SAD and GAD, which could have important theoretical and clinical implications for these disorders. 
Table 1

Demographic Characteristics of the Sample Stratified by Group

\begin{tabular}{|c|c|c|c|c|}
\hline & $\begin{array}{c}\text { SAD } \\
(n=21)\end{array}$ & $\begin{array}{c}\text { GAD } \\
(n=20)\end{array}$ & $\begin{array}{l}\text { Nonclinical } \\
\text { Comparison } \\
\quad(n=20)\end{array}$ & $\chi^{2}$ or $F$ \\
\hline \multicolumn{5}{|l|}{ Gender $n(\%)$} \\
\hline Female & $17(80.95)$ & $13(65)$ & $14(70)$ & \\
\hline Male & $3(14.29)$ & $7(35)$ & $6(30)$ & \\
\hline Transgender & $1(4.76)$ & $0(0)$ & $0(0)$ & \\
\hline Age $M(S D)$ & $22.08(5.35)$ & $23.50(8.82)$ & $21.83(1.64)$ & 1.35 \\
\hline Ethnicity $n(\%)$ & & & & 10.78 \\
\hline Asian & $6(28.57)$ & $10(50)$ & $10(50)$ & \\
\hline White & $11(52.38)$ & $8(40)$ & $5(25)$ & \\
\hline Black & $2(9.52)$ & $0(0)$ & $3(15)$ & \\
\hline Biracial/Multiracial & $2(9.52)$ & $1(5)$ & $1(5)$ & \\
\hline Hispanic & $0(0)$ & $0(0)$ & $1(5)$ & \\
\hline Other & $0(0)$ & $1(5)$ & $0(0)$ & \\
\hline Relationship Status $n(\%)$ & & & & 5.68 \\
\hline Single & $12(57.14)$ & $13(65)$ & $12(60)$ & \\
\hline Steady Relationship & $6(28.57)$ & $5(25)$ & $5(25)$ & \\
\hline Cohabiting & $2(9.52)$ & $1(5)$ & $1(5)$ & \\
\hline Married & $0(0)$ & $0(0)$ & $2(10)$ & \\
\hline Divorced & $1(4.76)$ & $1(5)$ & $0(0)$ & \\
\hline Enrolment: Yes (\%) & $13(61.90)$ & $13(65)$ & $12(60)$ & .12 \\
\hline Type of Program $n(\%)$ & & & & 2.06 \\
\hline University & $11(52.38)$ & $11(55)$ & $12(60)$ & \\
\hline Community College & $1(4.76)$ & $1(5)$ & $0(0)$ & \\
\hline Adult/Continuing & $1(4.67)$ & $1(5)$ & $0(0)$ & \\
\hline
\end{tabular}


Education Level $n(\%)$

Some HS

$1(4.76)$

$0(0)$

$0(0)$

Completed HS/Equivalency

$2(9.52)$

4 (20)

$2(10)$

Some College or University

10 (47.62)

$5(25)$

$10(50)$

Graduated College or

7 (33.33)

$7(35)$

$5(25)$

University

Employment Status $n(\%)$

.76

Not Working

$10(47.62)$

$10(50)$

$11(55)$

Part-Time

$9(47.86)$

9 (45)

$7(35)$

Full-Time

$2(9.52)$

$1(5)$

$2(10)$

Compliance Ratings $M(S D)$

79.52 (13.78)

$74.75(14.73)$

$83.90(15.82)$

1.92

Note. $\mathrm{SAD}=$ Social Anxiety Disorder; GAD = Generalized Anxiety Disorder; Nonclinical Comparison $=$ individuals with no lifetime history of mental health difficulties; Chi-square tests $\left(\chi^{2}\right)$ were conducted for Gender, Ethnicity, Relationship Status, Enrolment, Type of Program, Education Level, and Employment Status; ANOVA $(F)$ tests were conducted for Age and Compliance Ratings. 
Table 2

Descriptive Statistics for Frequency of Feedback Seeking Behaviours Over A 2-Week Period (Based on Diary Records) Stratified by Group

\begin{tabular}{|c|c|c|c|c|c|c|}
\hline \multicolumn{7}{|c|}{$\begin{array}{c}\text { SAD } \\
(n=21)\end{array}$} \\
\hline Variable & $M$ & $S D$ & Min & Max & Total & $\begin{array}{c}\% \text { of Total } \\
\text { (compliance) }\end{array}$ \\
\hline PFS & 5.67 & 3.67 & 0 & 14 & 119 & $64.32 \%$ \\
\hline NFS & .90 & 1.22 & 0 & 4 & 19 & $10.27 \%$ \\
\hline $\begin{array}{l}\text { Total FS (no } \\
\text { compliance) }\end{array}$ & 6.57 & 4.21 & 0 & 17 & 138 & $74.59 \%$ \\
\hline $\begin{array}{l}\text { Total FS } \\
\text { (compliance) }\end{array}$ & 8.81 & 5.69 & 0 & 21 & 185 & $100.00 \%$ \\
\hline \multicolumn{7}{|c|}{$\begin{array}{c}\text { GAD } \\
(n=20)\end{array}$} \\
\hline Variable & $M$ & $S D$ & Min & Max & Total & $\begin{array}{c}\% \text { of Total } \\
\text { (compliance) }\end{array}$ \\
\hline PFS & 7.90 & 4.54 & 1 & 16 & 158 & $59.40 \%$ \\
\hline NFS & 1.35 & 1.27 & 0 & 4 & 27 & $10.15 \%$ \\
\hline $\begin{array}{l}\text { Total FS (no } \\
\text { compliance) }\end{array}$ & 9.25 & 4.66 & 2 & 17 & 185 & $69.55 \%$ \\
\hline $\begin{array}{l}\text { Total FS } \\
\text { (compliance) }\end{array}$ & 13.30 & 7.62 & 2 & 27 & 266 & $100.00 \%$ \\
\hline
\end{tabular}

\begin{tabular}{|c|c|c|c|c|c|c|}
\hline \multicolumn{7}{|c|}{$\begin{array}{l}\text { Nonclinical Comparison } \\
\qquad(\mathrm{n}=20)\end{array}$} \\
\hline Variable & $M$ & $S D$ & Min & Max & Total & $\begin{array}{c}\% \text { of Total } \\
\text { (compliance) }\end{array}$ \\
\hline
\end{tabular}




\begin{tabular}{lcccccc} 
PFS & 5.85 & 4.72 & 0 & 17 & 117 & $71.78 \%$ \\
NFS & 1.05 & 1.47 & 0 & 5 & 21 & $12.88 \%$ \\
$\begin{array}{l}\text { Total FS (no } \\
\text { compliance) }\end{array}$ & 6.90 & 5.53 & 0 & 18 & 138 & $84.66 \%$ \\
$\begin{array}{l}\text { Total FS } \\
\text { (compliance) }\end{array}$ & 8.40 & 6.46 & 0 & 20 & 163 & $100.00 \%$ \\
\hline
\end{tabular}

Note. Min = Minimum number of diary records completed over a two-week period; Max = Maximum number of diary records completed over a two-week period; Total $=$ Total number of diary records completed over a two-week period; $\%$ of Total (compliance) $=$ Percentage of total feedback seeking behaviours (taking compliance ratings into account); PFS = Positive Feedback Seeking; NFS = Negative Feedback Seeking; Total FS (no compliance) = Total Feedback Seeking (based on completed records and therefore not taking compliance ratings into account); Total FS (compliance) = Total Feedback Seeking (taking compliance ratings into account); $\%$ of Total (compliance) $=\%$ of Total (taking compliance ratings into account). 
Table 3

Self-Report Questionnaire Scores Stratified by Group

\begin{tabular}{lcccc}
\hline & $\begin{array}{c}\text { SAD } \\
(n=21)\end{array}$ & $\begin{array}{c}\text { GAD } \\
(n=20)\end{array}$ & $\begin{array}{c}\text { Nonclinical } \\
\text { Comparison } \\
(n=20)\end{array}$ & $F$ or $H$ \\
\hline $\begin{array}{l}\text { GAD-Q-IV } M \\
(S D)\end{array}$ & $7.31(3.42)$ & $9.44(2.29)$ & $1.60(2.46)$ & $33.85^{* *}$ \\
PSWQ $M(S D)$ & $62.64(8.36)$ & $64.43(10.75)$ & $37.05(11.23)$ & $36.24^{* *}$ \\
SPIN $M(S D)$ & $43.24(8.46)$ & $28.15(8.60)$ & $8.23(7.02)$ & $45.94^{* *}$ \\
DASS-D $M(S D)$ & $14.00(10.70)$ & $19.70(8.66)$ & $4.20(4.89)$ & $24.89^{* *}$ \\
STICSA $M(S D)$ & $44.76(10.59)$ & $50.30(6.38)$ & $28.10(4.89)$ & $81.93^{* *}$ \\
IUS $M(S D)$ & $84.17(15.15)$ & $84.88(12.62)$ & $54.10(20.45)$ & $26.11^{* *}$ \\
BFNE $M(S D)$ & $29.57(7.17)$ & $31.85(4.65)$ & $15.10(6.44)$ & $43.22^{* *}$ \\
FPES $M(S D)$ & $35.81(18.89)$ & $32.20(16.99)$ & $17.05(12.56)$ & $11.70^{*}$ \\
\hline
\end{tabular}

Note. GAD-Q-IV = Generalized Anxiety Disorder Questionnaire; PSWQ = Penn State Worry Questionnaire; SPIN = Social Phobia Inventory; DASS-D = Depression Anxiety Stress Scales, 21-item version-Depression subscale; STICSA = State-Trait Inventory for Cognitive and Somatic Anxiety; IUS = Intolerance of Uncertainty Scale; BFNE = Brief Fear of Negative Evaluation Scale; FPES = Fear of Positive Evaluation Scale; ANOVA $(F)$ tests were conducted to evaluate group differences on the PSWQ, STICSA, and BFNE; Kruskal-Wallis $(H)$ tests were conducted to evaluate group differences on the GAD-Q-IV, SPIN, DASS-D, IUS, and FPES.

$* p<.01 ; * * p<.001$ 
Table 4

Regression Coefficients for Models Predicting Feedback Seeking Frequency from Group

\begin{tabular}{lccccc}
\hline Model 1: PFS & $\Delta R^{2}$ & $\Delta F$ & $\beta$ & $t$ & $p$ \\
\hline & & & & & \\
Step 1: SAD vs. Nonclinical Comparison & .05 & 1.66 & -.02 & -.14 & .89 \\
GAD vs. Nonclinical Comparison & & & .22 & 1.50 & .14 \\
& & & & & \\
\hline
\end{tabular}

Model 2: NFS

Step 1: SAD vs. Nonclinical Comparison $\quad .02$

GAD vs. Nonclinical Comparison

$.02 \quad .60$

$\begin{array}{llll}60 & -.05 & -.35 & .73\end{array}$

$.11 \quad .72 \quad .48$

Model 3: Overall FS (no compliance)

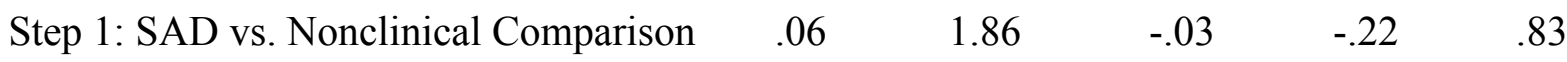

$\begin{array}{llll}\text { GAD vs. Nonclinical Comparison } & .23 & 1.54 & .13\end{array}$

Model 4: Overall FS (compliance)

Step 1: SAD vs. Nonclinical Comparison $\quad .10$

GAD vs. Nonclinical Comparison

$.10 \quad 3.36$

.03

.20

.84

$.34 \quad 2.33$

.02

Model 5: Overall FS (compliance)

Step 1: IUS

Step 2: GAD vs. Nonclinical Comparison

Step 3: Interaction

$\begin{array}{lllcl}.08 & 5.34 & .39 & 2.63 & .01 \\ .06 & 3.83 & .09 & .61 & .55 \\ .08 & 5.55 & .34 & 2.36 & .02\end{array}$

Note. PFS = Positive Feedback Seeking; NFS = Negative Feedback Seeking; Overall FS (no compliance) = Overall Feedback Seeking (based on completed diary records and therefore not taking compliance ratings into account); Overall FS (compliance) = Overall Feedback Seeking (taking compliance ratings into account); IUS = Intolerance of Uncertainty Scale scores; Interaction = Interaction between group (GAD vs. healthy) and Intolerance of Uncertainty Scale scores. 
Table 5

Correlations between Frequency of Feedback Seeking Behaviours Over a 2-Week Period (Based on Diary Records) and Scores on Feedback Seeking Questionnaires $(N=61)$

\begin{tabular}{lcccccccc}
\hline & $\begin{array}{c}\text { PFSF- } \\
\text { Q- } \\
\text { Social }\end{array}$ & $\begin{array}{c}\text { PFSF- } \\
\text { Q- } \\
\text { General }\end{array}$ & $\begin{array}{c}\text { PFSF- } \\
\text { Q-Other }\end{array}$ & $\begin{array}{c}\text { PFSF- } \\
\text { Q-Full }\end{array}$ & $\begin{array}{c}\text { NFSF- } \\
\text { Q- } \\
\text { Social }\end{array}$ & $\begin{array}{c}\text { NFSF- } \\
\text { Q- } \\
\text { General }\end{array}$ & $\begin{array}{c}\text { NFSF- } \\
\text { Q- } \\
\text { Other }\end{array}$ & $\begin{array}{c}\text { NFSF- } \\
\text { Q-Full }\end{array}$ \\
\hline PFS Diaries & .16 & $.26^{*}$ & .24 & $.25^{*}$ & .05 & -.05 & .05 & -.02 \\
NFS Diaries & $.45^{* * *}$ & .23 & $.42^{*}$ & $.37^{* *}$ & $.39 * *$ & $.27 *$ & $.40^{* *}$ & $.33^{*}$ \\
$\begin{array}{l}\text { Total FSDs } \\
\text { (No compliance) }\end{array}$ & .25 & $.27^{*}$ & $.31^{*}$ & $.30^{*}$ & .14 & .03 & .15 & .07 \\
$\begin{array}{l}\text { Total FSDs } \\
\text { (Compliance }\end{array}$ & $.32 *$ & $.30^{*}$ & $.34^{* *}$ & $.34 *$ & .22 & .11 & .25 & .15 \\
\hline
\end{tabular}

$\begin{array}{llll}\text { RSS- } & \text { RSS-Social } & \text { RSS-Threat } & \text { DIRI-RS }\end{array}$

\begin{tabular}{|c|c|c|c|c|c|}
\hline PFS Diaries & .11 & .14 & .03 & .17 & -.11 \\
\hline NFS Diaries & $.31 *$ & .21 & .13 & $.31^{*}$ & .18 \\
\hline $\begin{array}{l}\text { Total Diaries } \\
\text { (No compliance) }\end{array}$ & .18 & .18 & .06 & .21 & -.02 \\
\hline $\begin{array}{l}\text { Total Diaries } \\
\text { (Compliance) }\end{array}$ & .21 & .19 & .03 & .24 & .02 \\
\hline
\end{tabular}

Note. PFS Diaries = Positive Feedback Seeking Diaries; NFS Diaries = Negative Feedback Seeking Diaries; Total FSDs (no compliance = Total Feedback Seeking Diaries not adjusted for compliance; Total FSDs (Compliance) = Total Feedback Seeking Diaries adjusted for compliance; PFSF-Q-Social = Positive Feedback Seeking Frequency Questionnaire- Social subscale; PFSF-Q-General = Positive Feedback Seeking Frequency Questionnaire- General subscale; PFSF-Q-Other = Positive Feedback Seeking Frequency Questionnaire-Other subscale; PFSF-Q-Total = Positive Feedback Seeking Frequency Questionnaire- Full scale; NFSF-Q-Social = Negative Feedback Seeking Frequency Questionnaire- Social subscale; NFSFQ-General = Negative Feedback Seeking Frequency Questionnaire- General subscale; NFSF-QOther = Negative Feedback Seeking Frequency Questionnaire- Other subscale; NFSF-Q-Total = Negative Feedback Seeking Frequency Questionnaire- Full scale; RSS-Decision = Reassurance Seeking Scale-Decision-Making subscale: RSS-Social = Reassurance Seeking Scale-Social 
Attachment subscale; RSS-Threat $=$ Reassurance Seeking General Threat subscale; DIRI-RS = Depressive Interpersonal Relationships Inventory-Reassurance-Seeking Subscale; FSQ = Feedback Seeking Questionnaire.

$* p<.05 ; * * p<.01 ; * * *<.001$ 
Table 6

Mean Percentages of Topics of Overall Feedback Seeking Over a 2-Week Period (Based on Diary Records) Stratified by Group

\begin{tabular}{|c|c|c|c|c|c|}
\hline & $\begin{array}{c}\text { SAD } \\
(n=21)\end{array}$ & $\begin{array}{c}\text { GAD } \\
(n=20)\end{array}$ & $\begin{array}{l}\text { Nonclinical } \\
\text { Comparison } \\
\quad(n=20)\end{array}$ & $F$ & $p$ \\
\hline \multicolumn{6}{|c|}{ Social/Evaluative $M \%(S D)$} \\
\hline $\begin{array}{l}\text { Social skills or social } \\
\text { competence }\end{array}$ & $15.49(17.98)$ & $12.21(13.23)$ & $9.86(18.92)$ & .58 & .57 \\
\hline Physical appearance & $24.53(23.76)$ & $23.27(23.92)$ & $16.97(23.40)$ & .59 & .56 \\
\hline Signs of anxiety & $7.04(10.98)$ & $3.33(5.82)$ & $1.33(4.64)$ & 2.89 & .06 \\
\hline \multicolumn{6}{|l|}{ General $M \%(S D)$} \\
\hline Personal abilities & $18.34(16.26)$ & $19.51(15.27)$ & $20.99(23.94)$ & .10 & .90 \\
\hline Life achievements & $14.23(19.38)$ & $20.45(24.25)$ & $8.91(12.89)$ & 1.77 & .18 \\
\hline Decision-making & $25.02(23.29)$ & $27.56(26.73)$ & $23.57(26.45)$ & .13 & .88 \\
\hline Personal health & $11.96(19.93)$ & $13.34(14.58)$ & $3.76(6.21)$ & 2.45 & .10 \\
\hline Health of others & $6.02(13.93)$ & $3.48(8.11)$ & $1.71(5.36)$ & .99 & .38 \\
\hline Personal safety & $1.54(5.67)$ & $3.14(8.37)$ & $3.25(10.29)$ & .27 & .77 \\
\hline Safety of others & $6.85(12.22)$ & $.81(2.57)$ & $2.00(8.94)$ & 2.65 & .08 \\
\hline Financial security & $2.55(6.77)$ & $5.11(12.99)$ & $1.05(3.45)$ & 1.12 & .33 \\
\hline
\end{tabular}


Other $M \%(S D)$

Quality or security of relationships

13.04 (18.90)

$6.15(12.25)$

1.56

.22

Self-worth or lovability

$18.21(22.21)$

$17.77(3.93)$

$11.28(4.92)$

.71

.50

Level of performance

$30.82(25.84)$

$27.73(23.95)$

23.33 (18.63)

.54

.58

Intelligence

$9.70(13.07)$

12.54 (11.96)

6.99 (18.92)

.69

.51

Note. Social/Evaluative $=$ Topics that were rated as more characteristic of social anxiety than generalized anxiety by more than 75\% of raters; General = Topics that were rated as more characteristic of generalized anxiety than social anxiety by more than 75\% of raters; Other = Topics that fewer than 75\% of raters agreed were more characteristic of social anxiety or generalized anxiety; therefore, these topics may be characteristic of both social anxiety and generalized anxiety or of neither social anxiety or generalized anxiety. 
Table 7

Mean Percentages of Sources of Overall Feedback Seeking Over a 2-Week Period (Based on Diary Records) Stratified by Group

$\begin{array}{ccccc}\text { SAD } & \text { GAD } & \begin{array}{c}\text { Nonclinical } \\ \text { Comparison } \\ (n=21)\end{array} & F & p\end{array}$

Sources $M \%(S D)$

Romantic partner

$12.55(21.61) \quad 9.38(16.20) \quad 18.01(31.27) \quad .67 \quad .51$

Family member

$\begin{array}{lllll}27.63(36.17) & 18.14(24.64) & 16.53(17.54) & .99 & .38\end{array}$

Friend

$\begin{array}{lllll}29.19(34.50) & 46.64(30.50) & 25.19(28.08) & 2.68 & .08\end{array}$

Acquaintance

$\begin{array}{llllll}4.65(7.93) & 7.51(10.83) & 6.63(12.37) & .40 & .67\end{array}$

Stranger

$\begin{array}{lllll}3.57(6.52) & 3.28(6.33) & 1.05(3.45) & 1.22 & .30\end{array}$

Oneself

Professional/authority $12.52(25.83) \quad 4.80(12.47) \quad 1.39(4.56) \quad 2.32 \quad .12$ $\begin{array}{lllll}7.89(13.83) & 9.19(11.43) & 9.93(14.87) & .12 & .89\end{array}$

Sources other than people (e.g., websites)

Note. Individuals with SAD completed a total of 138 diary records; individuals with GAD completed a total of 185 diary records; and the nonclinical comparison group completed a total of 138 diary records. 
Table 8

Mean Percentages of Triggers of Positive Feedback Seeking, Negative Feedback Seeking, and

Overall Feedback Seeking Over a 2-Week Period (Based on Diary Records) Stratified by Group

$\begin{array}{ccccc}\text { SAD } & \text { GAD } & \begin{array}{c}\text { Nonclinical } \\ \text { Comparison } \\ (n=21)\end{array} & F & p\end{array}$

Triggers of PFS $M \%(S D)$

Social/evaluative

$56.47(34.74)$

$42.92(30.88)$

$37.93(33.46)$

1.73

.19

General

$30.33(31.01)$

$44.56(30.93)$

$33.24(35.12)$

$1.09 \quad .34$

Other

$7.96(14.95)$

$10.85(25.08)$

$13.83(26.90)$

.34

.71

Triggers of NFS $M \%(S D)$

Social/evaluative

General

Other

Triggers of Overall FS $M \%$ $(S D)$

Social/evaluative

General

Other
$27.78(40.25)$

$15.08(29.30)$

$.00(.00)$
$45.42(47.56)$

36.33 (46.82)

.79

.46

$16.25(34.67)$

$6.17(15.30)$

.80

.46

$8.33(26.21)$

$7.50(24.47)$

1.03

.36

Note. Individuals with SAD completed a total of 138 diary records; individuals with GAD completed a total of 185 diary records; and the nonclinical comparison group completed a total of 138 diary records. "Other" refers to triggers that were neither social/evaluative nor general. 
Table 9

Mean Percentages of Functions of Positive Feedback Seeking and Negative Feedback Seeking Over a 2-Week Period (Based on Diary Records) Stratified by Group

$\begin{array}{ccccc}\text { SAD } & \text { GAD } & \begin{array}{c}\text { Nonclinical } \\ \text { Comparison } \\ (n=21)\end{array} & F & p\end{array}$

Functions of PFS $M \%(S D)$

$\begin{array}{llllll}\text { Social/evaluative } & 56.95(34.57) & 39.01(31.25) & 37.01(33.61) & 2.26 & .11 \\ \text { General } & 30.81(30.60) & 48.29(32.04) & 33.95(34.58) & 1.68 & .20 \\ \text { Other } & 7.48(13.65) & 12.36(26.69) & 13.21(24.91) & .39 & .68\end{array}$

Functions of NFS $M \%(S D)$

$\begin{array}{lccccc}\text { Confirm } & 19.05(33.45) & 27.92(39.21) & 12.00(26.87) & 1.13 & .33 \\ \text { Avoid } & .00(.00) & .00(.00) & 1.25(5.59) & 1.03 & .37 \\ \text { Lower } & .00(.00) & .00(.00) & .00(.00) & & \\ \text { Other } & 23.81(37.48) & 42.08(44.55) & 36.75(45.72) & 1.00 & .37\end{array}$

Note. Individuals with SAD completed 119 PFS diary records and 19 NFS diary records; individuals with GAD completed 158 PFS diary records and 27 NFS diary records; and the nonclinical comparison group completed 117 PFS diary records and 21 NFS diary records. Confirm = Confirm negative self views; Avoid = Avoid appearing "too good" in front of others; Lower = Lower others' expectations of oneself; Other = Other functions of feedback seeking (e.g., desire for improvement). 
Table 10

Mean Percentages of Termination Criteria of Positive Feedback Seeking and Negative Feedback Seeking Over a 2-Week Period (Based on Diary Records) Stratified by Group

$\begin{array}{ccccc}\text { SAD } & \text { GAD } & \begin{array}{c}\text { Nonclinical } \\ \text { Comparison } \\ (n=21)\end{array} & F & p\end{array}$

Termination of PFS $M \%(S D)$

Social/evaluative

General

Other

$\begin{array}{lllll}35.48(30.38) & 25.55(25.23) & 33.45(32.64) & .64 & .53 \\ 17.95(23.41) & 28.21(25.15) & 28.88(31.93) & 1.06 & .35 \\ 41.81(29.15) & 45.57(29.80) & 22.67(26.68) & 3.71 & .03\end{array}$

Termination of NFS $M \%$ $(S D)$

$\begin{array}{lcccrr}\text { Confirm } & 4.76(15.04) & 7.50(24.47) & 2.50(11.18) & .40 & .67 \\ \text { Avoid } & .00(.00) & .00(.00) & .00(.00) & & \\ \text { Lower } & .00(.00) & .00(.00) & .00(.00) & & \\ \text { Other } & 38.09(47.19) & 62.50(48.33) & 47.50(49.93) & 1.32 & .28\end{array}$

Note. Individuals with SAD completed 119 PFS diary records and 19 NFS diary records; individuals with GAD completed 158 PFS diary records and 27 NFS diary records; and the nonclinical comparison group completed 117 PFS diary records and 21 NFS diary records. Confirm = Confirm negative self views; Avoid = Avoid appearing "too good" in front of others; Lower = Lower others' expectations of oneself; Other = Other functions of feedback seeking (e.g., desire for improvement). 
Table 11

Mean Pre-, Post-, and Change Scores on Affective States, Certainty, and Uncertainty After Receiving Positive Feedback During Episodes of Positive Feedback Seeking

$\begin{array}{cccc}\text { SAD } & \text { GAD } & \text { Nonclinical } & \\ (n=21) & (n=20) & \begin{array}{c}\text { Comparison } \\ (n=20)\end{array} & \end{array}$

Fearful $M(S D)$

Pre $\quad 1.96(1.37) \quad 1.83(1.28) \quad .84(1.12)$

Post $\quad .84(1.13) \quad 1.06(.94) \quad .37(.69)$

$\begin{array}{llllll}\text { Change } & -1.12(1.15) & -.77(.77) & -.47(.93) & 1.97 & .15\end{array}$

Anxious $M(S D)$

$\begin{array}{llllll}\text { Pre } & 3.02(1.32) & 3.01(1.26) & 1.15(1.34) & \\ \text { Post } & .96(1.17) & 1.46(1.06) & .41(.67) & & \\ \text { Change } & -2.07(1.13) & -1.55(1.17) & -.74(1.16) & 5.64 & .01\end{array}$

$\operatorname{Sad} M(S D)$

$\begin{array}{llllll}\text { Pre } & 1.70(1.23) & 1.56(1.38) & .64(.81) & \\ \text { Post } & .83(1.09) & 1.11(1.18) & .43(.71) & & \\ \text { Change } & -.87(.75) & -.45(.67) & -.21(.58) & 4.23 & .02\end{array}$

Uncertain $M(S D)$

\begin{tabular}{|c|c|c|c|c|}
\hline Pre & $3.59(1.20)$ & $3.42(1.24)$ & $2.12(1.38)$ & \\
\hline Post & $1.01(1.07)$ & $1.32(.99)$ & $.50(.62)$ & \\
\hline Change & $-2.58(1.16)$ & $-2.11(1.36)$ & $-1.63(1.36)$ & 2.29 \\
\hline
\end{tabular}


Frustrated $M(S D)$

$\begin{array}{llllll}\text { Pre } & 1.77(1.42) & 1.85(1.22) & .84(.74) & & \\ \text { Post } & .79(1.12) & 1.03(1.03) & .49(.66) & & \\ \text { Change } & -.98(1.02) & -.82(.86) & -.35(.80) & 2.22 & .12\end{array}$

Disgusted $M(S D)$

$\begin{array}{llll}\text { Pre } & .82(1.22) & .55(.83) & .20(.48) \\ \text { Post } & .59(1.02) & .31(.61) & .27(.54)\end{array}$

Change

$\begin{array}{llllll}-.23 & -.24 & .07 & 2.10 & .13\end{array}$

Angry $M(S D)$

Pre

$\begin{array}{lll}.86(1.10) & .72(.86) \quad .19(.40)\end{array}$

Post

$\begin{array}{lll}.50(.83) & .29(.70) \quad .28(.54)\end{array}$

Change

$\begin{array}{lll}-.37(.59) & -.42(.56) & .09(.30)\end{array}$

$5.11 \quad .01$

Ashamed $M(S D)$

$\begin{array}{llllll}\text { Pre } & .95(1.14) & .92(.97) & .26(.40) & & \\ \text { Post } & .45(.75) & .59(.81) & .24(.53) & & \\ \text { Change } & -.50(.80) & -.33(.72) & -.02(.31) & 3.34 & .05\end{array}$

Guilty $M(S D)$

$\begin{array}{llllll}\text { Pre } & 1.30(1.42) & .72(1.08) & .42(.51) & & \\ \text { Post } & 1.05(1.32) & .72(.77) & .28(.53) & & \\ \text { Change } & -.25(1.02) & -.01(.62) & -.13(.55) & .46 & .64\end{array}$

Certain $M(S D)$
Pre
$.97(.72)$
$1.26(1.19)$
$2.18(1.44)$
Post
$3.36(1.32) \quad 2.58(1.58) \quad 3.77(1.48)$ 
Change

Confident $M(S D)$

Pre

Post

Change

Relaxed $M(S D)$

Pre

Post

Change

Happy $M(S D)$

Pre

$1.60(1.23) \quad 1.35(1.34) \quad 2.49(1.77)$

Post

$3.40(1.27) \quad 2.48(1.69) \quad 4.26(1.44)$

Change
$2.39(1.35)$

$1.31(1.13)$

$1.59(1.45)$

3.36

.04

$1.30(.95) \quad 1.39(1.29) \quad 2.56(1.63)$

$3.52(1.06) \quad 2.68(1.68) \quad 4.02(1.40)$

$2.22(1.33)$

$1.29(1.40)$

$1.46(1.51)$

2.25

.12

$1.44(1.10) \quad 1.22(1.10) \quad 2.64(1.89)$

$3.25(1.37) \quad 2.57(1.63) \quad 3.96(1.63)$

$1.82(1.16) \quad 1.35(1.30) \quad 1.32(1.56)$

.77

.47

$1.39 \quad .26$

Note . Pre = Prior to seeking feedback; Post $=$ After receiving feedback; Change $=$ Change from prior to seeking feedback to after receiving feedback. 
Table 12

Mean Pre-, Post-, and Change Scores on Affective States, Certainty, and Uncertainty After Receiving Negative Feedback During Episodes of Positive Feedback Seeking

$\begin{array}{cccc}\text { SAD } & \text { GAD } & \text { Nonclinical } \\ (n=21) & (n=20) & \begin{array}{c}\text { Comparison } \\ (n=20)\end{array} & \end{array}$

Fearful $M(S D)$

$\begin{array}{llllll}\text { Pre } & 1.83(1.59) & 1.77(.95) & .44(.50) & \\ \text { Post } & 2.22(2.17) & 2.22(1.62) & .70(1.10) & \\ \text { Change } & .40(1.60) & .45(1.32) & .26(.91) & .03 & .97\end{array}$

Anxious $M(S D)$

$\begin{array}{llllll}\text { Pre } & 2.92(1.35) & 2.72(1.10) & .78(1.04) & & \\ \text { Post } & 2.56(2.24) & 2.75(2.23) & 1.00(1.41) & & \\ \text { Change } & .36(2.13) & .03(1.86) & .23(.88) & .19 & .83\end{array}$

$\operatorname{Sad} M(S D)$

\begin{tabular}{|c|c|c|c|c|}
\hline Pre & $2.25(1.23)$ & $1.71(1.44)$ & $.25(.48)$ & \\
\hline Post & $3.44(1.88)$ & $2.04(2.32)$ & $2.70(2.68)$ & \\
\hline Change & $1.20(2.19)$ & $.33(1.36)$ & $2.46(2.72)$ & 1.88 \\
\hline
\end{tabular}

Uncertain $M(S D)$

$\begin{array}{llllll}\text { Pre } & 3.36(1.44) & 3.26(1.08) & 1.89(1.69) & & \\ \text { Post } & 3.89(1.83) & 3.14(2.16) & 1.70(1.64) & & \\ \text { Change } & .53(2.42) & -.12(1.68) & -.19(1.20) & .34 & .72\end{array}$


Frustrated $M(S D)$

$\begin{array}{lccccc}\text { Pre } & 2.40(1.27) & 2.16(1.10) & 1.12(.96) & & \\ \text { Post } & 2.78(2.28) & 2.32(1.95) & 1.67(1.89) & & \\ \text { Change } & .38(2.67) & .16(1.63) & .54(2.28) & .06 & .95\end{array}$

Disgusted $M(S D)$

$\begin{array}{llllll}\text { Pre } & 1.16(1.34) & .46(.66) & .04(.09) & & \\ \text { Post } & 1.78(2.68) & .76(.80) & .23(.32) & & \\ \text { Change } & .62(1.46) & .30(.83) & .19(.26) & .38 & .69\end{array}$

Angry $M(S D)$

$\begin{array}{llllll}\text { Pre } & 1.20(1.02) & .97(1.02) & .09(.13) & & \\ \text { Post } & 1.44(2.07) & 1.76(1.86) & .90(1.24) & & \\ \text { Change } & .24(1.35) & .79(1.60) & .82(1.21) & .42 & .66\end{array}$

Ashamed $M(S D)$

$\begin{array}{llllll}\text { Pre } & 1.41(1.65) & 1.06(1.09) & .09(.10) & & \\ \text { Post } & 3.00(2.45) & 1.74(1.53) & .53(.77) & & \\ \text { Change } & 1.59(1.50) & .68(1.07) & .44(.72) & 1.97 & .17\end{array}$

Guilty $M(S D)$

$\begin{array}{lccccc}\text { Pre } & 1.51(1.68) & 1.00(1.33) & .19(.19) & & \\ \text { Post } & 2.22(2.39) & 1.52(1.88) & .43(.66) & & \\ \text { Change } & .71(1.50) & .52(1.28) & .24(.58) & .225 & .80\end{array}$

Certain $M(S D)$

$\begin{array}{llll}\text { Pre } & .84(.73) & 1.47(1.39) & 1.83(1.35) \\ \text { Post } & .78(1.72) & 1.60(1.78) & 1.73(1.69)\end{array}$



Change
$-.07(1.69)$
$.13(.71)$
$.10(1.44)$
.074
.93

Confident $M(S D)$

$\begin{array}{llll}\text { Pre } & 1.12(.79) & 1.70(1.60) & 2.27(1.43) \\ \text { Post } & .67(1.12) & 1.32(1.84) & 1.90(1.25)\end{array}$

Change

$-.46(.97) \quad-.38(.81) \quad-.37(.67)$

.026

.98

Relaxed $M(S D)$

Pre

$1.08(.84) \quad 1.39(1.30) \quad 2.52(1.55)$

Post

$1.22(1.72) \quad 1.38(1.71) \quad 1.53(1.45)$

Change

$\begin{array}{lll}.14(1.71) & -.01(1.01) \quad-.99(.97)\end{array}$

1.29

.30

Happy $M(S D)$

Pre

$1.24(.97) \quad 1.64(1.57) \quad 2.56(1.54)$

Post

$.67(1.00) \quad 1.24(1.75) \quad 2.00(1.41)$

Change

$-.58(1.06) \quad-.40(.78) \quad-.56(.99)$

.09

.91

Note. Pre $=$ Prior to seeking feedback; Post $=$ After receiving feedback; Change $=$ Change from prior to seeking feedback to after receiving feedback. 
Table 13

Mean Pre-, Post-, and Change Scores on Affective States, Certainty, and Uncertainty After Receiving Positive Feedback During Episodes of Negative Feedback Seeking

$\begin{array}{cccc}\text { SAD } & \text { GAD } & \text { Nonclinical } \\ (n=21) & (n=20) & \begin{array}{c}\text { Comparison } \\ (n=20)\end{array} & \end{array}$

Fearful $M(S D)$

$\begin{array}{llllll}\text { Pre } & 3.00(2.00) & 2.03(1.57) & 2.69(.90) & & \\ \text { Post } & 1.67(1.53) & 1.00(1.41) & 1.58(1.34) & \\ \text { Change } & -1.33(1.53) & -1.03(1.00) & -1.10(1.85) & .04 & .96\end{array}$

Anxious $M(S D)$

$\begin{array}{lllll}\text { Pre } & 4.33(1.61) & 3.00(1.41) & 3.38(1.36) & \\ \text { Post } & 3.17(1.76) & 2.00(1.87) & 1.42(1.26) & \\ \text { Change } & -1.17(1.26) & -1.00(2.12) & -1.96(1.01) & .41\end{array}$

$\operatorname{Sad} M(S D)$

$\begin{array}{lllll}\text { Pre } & 3.75(2.38) & 1.93(1.83) & 2.69(2.27) & \\ \text { Post } & 4.00(1.00) & 1.00(1.41) & 1.58(1.71) & \\ \text { Change } & .25(1.64) & .93(1.30) & -1.10(1.19) & 1.00\end{array}$

Uncertain $M(S D)$

\begin{tabular}{|c|c|c|c|c|}
\hline Pre & $4.00(1.00)$ & $3.07(.95)$ & $3.50(1.14)$ & \\
\hline Post & $3.67(1.15)$ & $1.00(1.73)$ & $1.08(1.34)$ & \\
\hline Change & $-.33(1.53)$ & $-2.07(1.25)$ & $-2.42(1.85)$ & 1.77 \\
\hline
\end{tabular}


Frustrated $M(S D)$

$\begin{array}{llllll}\text { Pre } & 4.67(1.53) & 2.77(1.67) & 3.25(2.10) & & \\ \text { Post } & 3.33(1.44) & 1.60(1.67) & 1.50(1.73) & & \\ \text { Change } & -1.33(1.04) & -1.17(1.28) & -1.75(1.19) & .27 & .77\end{array}$

Disgusted $M(S D)$

$\begin{array}{llll}\text { Pre } & 2.17(3.33) & 1.77(1.71) & 1.94(1.78) \\ \text { Post } & 1.83(2.75) & .60(1.34) & 1.50(1.73)\end{array}$

$\begin{array}{llllll}\text { Change } & -.33(.58) & -1.17(2.09) & -.44(.88) & .32 & .74\end{array}$

Angry $M(S D)$

Pre $\quad 1.67(.58) \quad 1.33(1.03) \quad 2.06(2.16)$

$\begin{array}{llll}\text { Post } & 1.67(1.15) & .40(.55) & 1.50(1.73)\end{array}$

$\begin{array}{llllll}\text { Change } & .00(1.73) & -.93(.92) & -.56(.66) & .67 & .53\end{array}$

Ashamed $M(S D)$

$\begin{array}{llllll}\text { Pre } & 4.17(2.36) & 1.77(1.20) & 2.44(1.90) & & \\ \text { Post } & 3.33(3.06) & 1.10(1.24) & 1.75(1.71) & & \\ \text { Change } & -.83(.76) & -.67(1.30) & -.69(.47) & .03 & .97\end{array}$

Guilty $M(S D)$

$\begin{array}{lccccc}\text { Pre } & 2.67(3.06) & 1.10(1.24) & 2.38(2.42) & & \\ \text { Post } & 2.83(3.01) & 1.00(1.73) & 1.17(1.91) & & \\ \text { Change } & .17(.29) & -.10(.74) & -1.21(.60) & 5.28 & .03\end{array}$

Certain $M(S D)$

$\begin{array}{llll}\text { Pre } & .92(1.18) & .40(.89) & 1.00(.82) \\ \text { Post } & 1.67(1.44) & 1.60(1.52) & 3.50(1.23)\end{array}$


$\begin{array}{llllll}\text { Change } & .75(1.09) & 1.20(1.64) & 2.50(1.04) & 1.68 & .24\end{array}$

Confident $M(S D)$

$\begin{array}{llllll}\text { Pre } & .92(1.18) & .80(1.79) & 1.31(1.03) & \\ \text { Post } & 2.00(1.80) & 2.20(2.05) & 3.50(1.23) & \\ \text { Change } & 1.08(1.01) & 1.40(2.41) & 2.19(1.15) & .83 & .48\end{array}$

Relaxed $M(S D)$

$\begin{array}{llllll}\text { Pre } & 1.33(1.04) & .60(.89) & 1.50(1.29) & & \\ \text { Post } & 1.00(.87) & 2.00(2.00) & 3.58(.96) & & \\ \text { Change } & -.33(1.15) & 1.40(1.95) & 2.08(1.50) & 1.90 & .21\end{array}$

Happy $M(S D)$

Pre $\quad 1.58(.63) \quad .80(1.30) \quad 1.25(1.50)$

Post $\quad 1.83(1.04) \quad 3.20(2.17) \quad 3.58(1.34)$

$\begin{array}{llllll}\text { Change } & .25(.43) & 2.40(2.51) & 2.33(.82) & 1.65\end{array}$

Note. Pre = Prior to seeking feedback; Post = After receiving feedback; Change $=$ Change from prior to seeking feedback to after receiving feedback. 
Table 14

Mean Pre-, Post-, and Change Scores on Affective States, Certainty, and Uncertainty After Receiving Negative Feedback During Episodes of Negative Feedback Seeking

$\begin{array}{cccc}\text { SAD } & \text { GAD } & \text { Nonclinical } \\ (n=21) & (n=20) & \begin{array}{c}\text { Comparison } \\ (n=20)\end{array} & \end{array}$

Fearful $M(S D)$

Pre $\quad 1.30(1.20) \quad 2.40(1.83) \quad 1.54(1.03)$

Post $\quad .90(2.01) \quad 2.09(2.67) \quad .79(.93)$

$\begin{array}{lllllll}\text { Change } & -.40(1.98) & -.30(1.52) & -.75(1.57) & .13 & .88\end{array}$

Anxious $M(S D)$

$\begin{array}{llllll}\text { Pre } & 2.80(1.79) & 3.35(1.39) & 2.33(.38) & \\ \text { Post } & 2.00(2.35) & 2.70(2.10) & 1.29(1.49) & \\ \text { Change } & -.80(1.10) & -.66(1.05) & -1.04(1.26) & .20 & .82\end{array}$

$\operatorname{Sad} M(S D)$

Pre $\quad 2.00(2.32) \quad 2.27(1.56) \quad 1.71(1.10)$

Post $\quad 2.60(2.41) \quad 3.03(1.81) \quad 1.29(1.49)$

$\begin{array}{lllllll}\text { Change } & .60(1.92) & .76(1.69) & -.42(1.55) & .89 & .43\end{array}$

Uncertain $M(S D)$

$\begin{array}{llllll}\text { Pre } & 3.30(.67) & 3.27(1.35) & 3.33(.89) & \\ \text { Post } & 3.20(1.64) & 3.27(1.71) & 3.04(1.40) & & \\ \text { Change } & -.10(1.60) & .00(1.54) & -.29(1.78) & .06 & .95\end{array}$


Frustrated $M(S D)$

$\begin{array}{lccccc}\text { Pre } & 2.80(1.04) & 3.18(1.43) & 3.75(.94) & & \\ \text { Post } & 2.80(1.79) & 2.68(1.90) & 2.75(2.36) & & \\ \text { Change } & .00(1.27) & -.50(2.09) & -1.00(2.41) & .33 & .72\end{array}$

Disgusted $M(S D)$

$\begin{array}{lccccc}\text { Pre } & 1.50(1.87) & 2.01(1.47) & 1.29(1.71) & & \\ \text { Post } & 1.80(2.05) & 2.06(2.08) & 1.92(2.54) & & \\ \text { Change } & .30(.45) & .05(2.26) & .63(1.30) & .20 & .82 \\ \text { ngry } M(S D) & & & & & \\ \text { Pre } & 1.60(1.67) & 1.58(1.19) & 1.46(1.82) & & \\ \text { Post } & 1.50(1.66) & 1.33(1.49) & 2.08(2.73) & & \\ \text { Change } & -.10(.74) & -.25(1.08) & .63(1.56) & 1.00 & .39\end{array}$

Ashamed $M(S D)$

$\begin{array}{llllll}\text { Pre } & 1.60(2.04) & 2.94(1.30) & 1.71(1.57) & & \\ \text { Post } & 1.40(1.95) & 2.83(1.71) & 1.92(1.80) & & \\ \text { Change } & -.20(1.15) & -.10(2.02) & .21(1.72) & .09 & .92\end{array}$

Guilty $M(S D)$

$\begin{array}{llllll}\text { Pre } & 1.20(1.79) & 1.22(.99) & 1.50(1.54) & & \\ \text { Post } & .60(1.34) & 2.82(1.92) & 2.00(1.67) & & \\ \text { Change } & -.60(.89) & 1.60(1.64) & .50(1.64) & 3.44 & .06\end{array}$

Certain $M(S D)$

$\begin{array}{llll}\text { Pre } & 1.35(.65) & .79(1.05) & 1.08(1.02) \\ \text { Post } & 2.30(1.57) & 1.77(1.94) & 1.58(1.80)\end{array}$


$\begin{array}{lllllll}\text { Change } & .95(1.50) & .98(1.89) & .50(1.38) & .17 & .85\end{array}$

Confident $M(S D)$

$\begin{array}{llllll}\text { Pre } & 1.05(1.07) & .90(1.39) & 1.63(1.14) & \\ \text { Post } & 1.20(1.30) & 1.03(.97) & 1.50(1.05) & \\ \text { Change } & .15(1.50) & .14(1.16) & -.13(.21) & .13 & .88\end{array}$

Relaxed $M(S D)$

$\begin{array}{llllll}\text { Pre } & 1.20(1.04) & .91(1.34) & 1.42(1.02) & \\ \text { Post } & 1.80(1.64) & 1.22(1.28) & 1.67(1.21) & \\ \text { Change } & .60(1.78) & .31(.96) & .25(1.08) & .12 & .89\end{array}$

Happy $M(S D)$

Pre $\quad 1.05(1.07) \quad .88(1.46) \quad 1.25(1.08)$

Post $\quad 1.50(1.41) \quad .77(1.36) \quad 1.63(1.34)$

$\begin{array}{llllll}\text { Change } & .45(1.57) & -.10(1.00) & .38(1.07) & .44 & .65\end{array}$

Note. Pre $=$ Prior to seeking feedback; Post $=$ After receiving feedback; Change $=$ Change from prior to seeking feedback to after receiving feedback. 


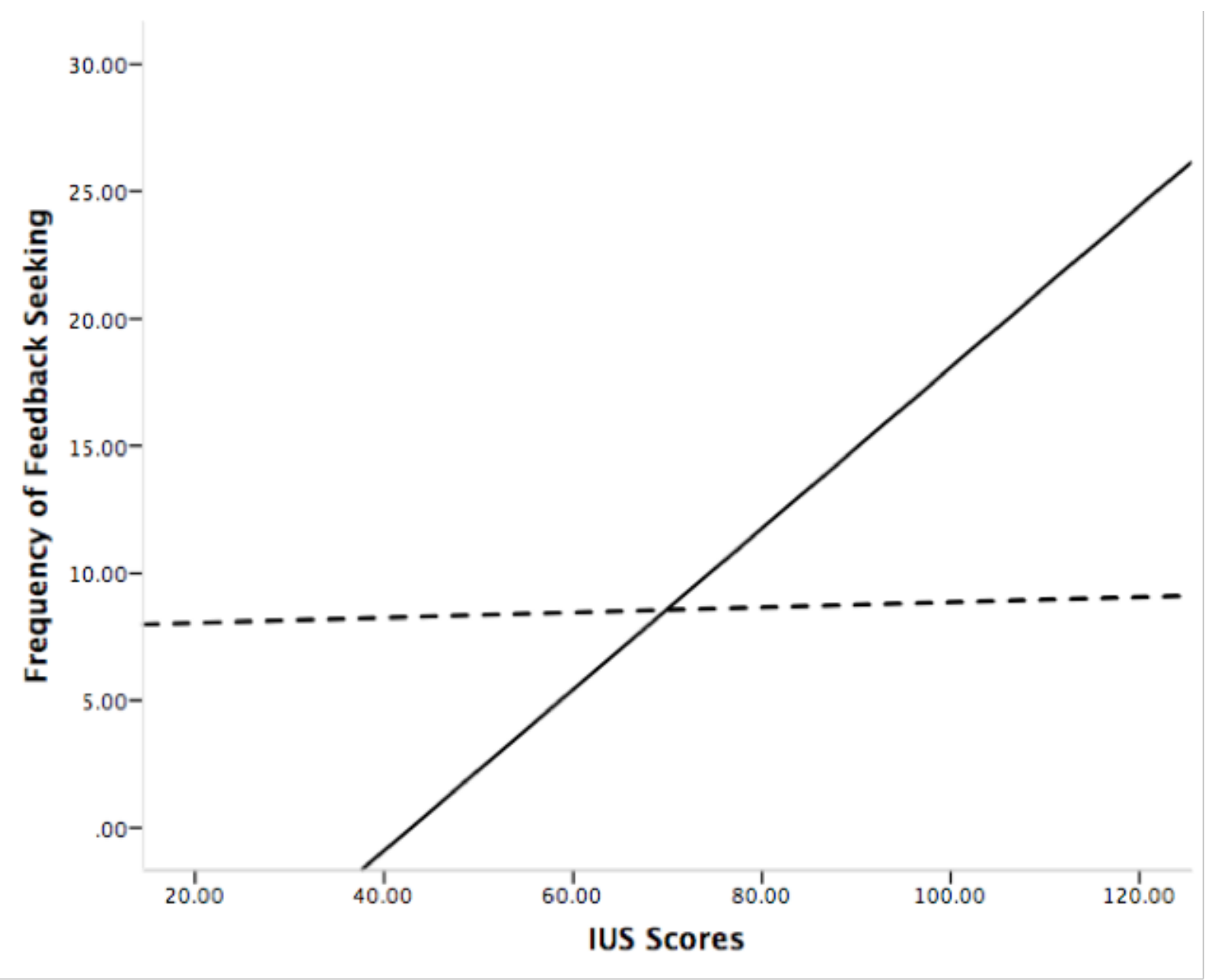

Figure 1. Regression lines of the predicted frequency of overall feedback seeking (adjusted for compliance ratings) from Intolerance of Uncertainty Scale scores stratified by group (GAD group versus nonclinical comparison group). 


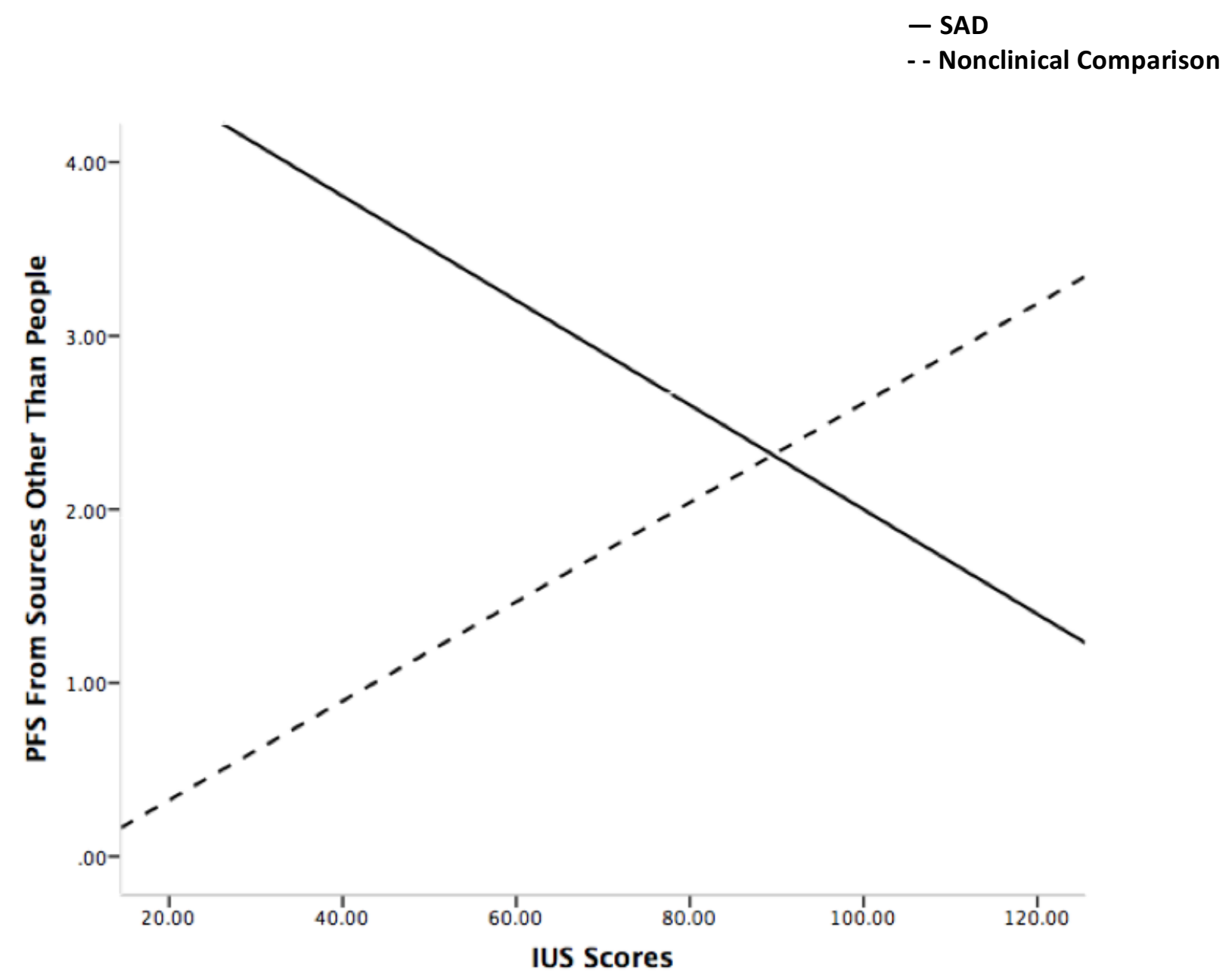

Figure 2. Regression lines of the predicted frequency of positive feedback seeking from sources other than people (e.g., websites, books) from Intolerance of Uncertainty Scale scores stratified by group (SAD group versus nonclinical comparison group). 
Appendix A: Telephone Screening Interview

$\underline{\text { Telephone Screening Interview }}$

Date of Interview:

Interviewer:

Participant ID Number: Email:

Home Telephone: Work Telephone:

\section{1) INTRODUCTION}

Hello, can I please speak to ? My name is and I am calling from the Anxiety Research and Treatment Lab at Ryerson University about your interest in a study being conducted in the lab. Are you interested in learning more about this study?

IF YES: Proceed to Study Description.

IF NO: Thank you very much for your time.

2) CONSENT AGREEMENT SCRIPT

Before I begin the interview to determine your eligibility for the study, I am going to read the following consent agreement so that you understand what your participation will involve. You will have the opportunity to ask any questions before you consent to participate in this interview.

This study is looking at feedback seeking in individuals with high social anxiety compared to individuals with high worry and individuals with no current or past mental health difficulties. Feedback seeking behaviours include seeking positive or negative information from others (e.g., partner, family members), oneself (e.g., reassuring self-talk), or external resources (e.g., websites, books). Some examples of seeking positive feedback include: 1) asking your classmate how you did on a presentation with the hope that he will provide reassurance that you did well, 2) asking your partner if he/she really loves you with the hope that your partner will affirm their love for you, and 3) asking your friend if you made the right decision with the hope that she will reassure you that you did. Some examples of seeking negative feedback include: 1) asking your classmate how you did on a presentation with the hope that he will tell you that you did poorly on the presentation (e.g., you were stuttering, seemed unprepared), 2) asking your partner if he/she really loves you with the hope that your partner will express dislike or hostility towards you, and 3) asking your friend if you made the correct decision with the hope that she will inform you that you made the wrong decision.

This study has several components, requiring participants to visit the lab on two separate occasions. Following this phone interview, eligible participants will be asked to come to the Anxiety Research and Treatment Lab on Ryerson campus. Upon arrival to the lab, participants will be asked to provide demographic information and to complete a packet of self-report questionnaires about anxiety (e.g., "Parties and social events scare me"), worry (e.g., "I am always worrying about something"), depression (e.g., "I couldn't seem to experience any positive feeling at all"), anxiety-related thoughts (e.g., "My mind can't be relaxed if I don't know what will happen tomorrow") and emotions (e.g., "I feel uneasy when I receive praise from authority figures"), and feedback seeking behaviours (e.g., "Do you find yourself often asking the people you feel close to how they truly feel about you?"). Participants will then be shown a diary record that they will be asked to complete every time they seek positive or 
negative feedback over a 2-week period. This in-lab component of the study will take approximately 1 hour to complete.

During the 2 weeks of completing diary records, participants will be sent a daily email reminder to help them accurately complete the records. After the 2 weeks, participants will come to the Anxiety Research and Treatment Lab on Ryerson campus for a second time, at which point they will be debriefed or given an in-depth explanation of the purpose the study, which has previously been described, and will have the opportunity to ask questions and express any concerns about the study. Individuals will be offered a \$30 incentive for their participation at this visit. This part of the study is expected to take approximately 30 minutes to complete.

There may be no direct benefits to you for completing this telephone interview. However, participants may benefit from gaining increased awareness into their anxiety-related thoughts, emotions, and behaviours. This research may also be important for increasing researchers' and clinicians' understanding of how feedback seeking behaviours manifest in the context of social anxiety, high worry, and no lifetime history of mental health problems. There is minimal risk involved in this task. By agreeing to complete this phone interview, you understand that you may experience some discomfort due to the personal nature of some of the questions asked in the interview. As such, the interview will be completed in the Anxiety Research and Treatment lab where your answers will not be overheard by anyone other than myself, the interviewer. Due to the personal nature of some of these questions, it is also important that you are currently in a place where others cannot overhear your answers.

If you feel uncomfortable answering any questions, you may skip the question or stop participation, as participation is completely voluntary. If you choose to stop participating, you may also choose to not have your data included in the study. Your choice of whether or not to participate will not influence your future relations with Ryerson University or the investigators involved in the research. If you choose to withdraw from participating during this telephone interview, however, you will not receive the \$30 incentive since this interview is part of the screening process to determine eligibility for the study. As previously mentioned, this incentive will be offered at the second lab visit. However, participants who voluntarily withdraw during the first visit to the lab or during the diary-recording phase of the study will still be entitled to the full $\$ 30$ incentive.

All data we collect from you is completely confidential, including the data collected during the phone interview, and will be identified with a participant ID number. Data will be collected and stored in password-protected files or locked in filing cabinets in the lab, to which only the primary investigator, research supervisor, and trained research assistants will have access. There are, however, certain conditions under which information must be reported to legal authorities without your consent: if you are at risk of harming yourself or another person, if you disclose information indicating that a child's welfare is in danger, or if you disclose information that a healthcare professional in Ontario has acted in a sexually inappropriate way toward you. In addition, if a court were to subpoena your record, then your file could be forwarded to the court.

Further, online data collected through the diary records will be entered into a computer using software called Qualtrics. The data are securely and confidentially stored on a remote server and you will be identified by number only. Please note that because the data is securely stored on a USA based server (Qualtrics), it is subject to the Patriot Act. If you would like to know more about this, please visit the following link: http://epic.org/privacy/terrorism/hr3162.html. Under the Patriot Act, stored data may be 
intercepted in rare cases if United States government officials have a reason to believe the data contains information related to suspected terrorism. However, your name is not stored with your questionnaire data, and therefore would not be available to these officials.

Do you have any questions about the study or anything that I just reviewed?

Your oral consent to participate in this interview indicates that you understand the contents just reviewed and have had a chance to ask any questions you have about the study. Your consent also indicates that your questions have been answered to your satisfaction, you agree to participate, and you have been told that you can withdraw at any time. Further, you understand that you can decide whether or not you would like to be contacted for future studies in the lab by answering the following question:

Would you like to be contacted for future studies in the Anxiety Research and Treatment Lab and/or the Cognition and Psychopathology Lab? YES NO Are you interested in participating in this study?

IF NO: Thank you very much for your time.

IF YES: Are you available for about 30 minutes to answer some questions now to determine your eligibility?

IF NO: Ask individual if he or she is willing to answer these questions at a later date. If so, schedule a phone interview on this date.

IF YES: Proceed to Inclusion/Exclusion Criteria.

\section{(a) Initial Screen}

1) Are you at least 18 years of age? YES NO

2) Do you have daily Internal access? YES NO

3) How would you rate your English language abilities? Poor Fair Good Excellent

IF YES TO \#1 AND \#2, AND GOOD TO EXCELLENT ON \#3: Proceed to Feedback Seeking Screen.

IF NO TO EITHER \#1 OR \#2, OR FAIR OR LESS ON \#3: Unfortunately, you are not eligible to participate in this study. Thank you for your interest and for taking the time to answer those questions.

\section{(b) Feedback Seeking Screen}

Now I'd like to ask you some questions to determine your eligibility to participate in this study...

\begin{tabular}{|c|c|}
\hline$\underline{\text { Question }}$ & Response: \\
& No (N); Yes (Y) \\
\hline
\end{tabular}




\begin{tabular}{|c|c|c|}
\hline $\begin{array}{l}\text { In the last } 6 \text { months, have you sought positive information or } \\
\text { reassurance from others, yourself (e.g., soothing self-talk), or } \\
\text { external sources (e.g., books)? Some examples of seeking positive } \\
\text { information include: 1) asking your classmate how you did on a } \\
\text { presentation with the hope that he will provide reassurance that you } \\
\text { did well, 2) asking your partner if he/she really loves you with the } \\
\text { hope that your partner will affirm their love for you, and 3) asking } \\
\text { your friend if you made the right decision with the hope that she will } \\
\text { reassure you that you did. } \\
\text { IF YES: Please explain: }\end{array}$ & $\mathrm{N}$ & $\mathrm{Y}$ \\
\hline $\begin{array}{l}\text { In the last } 6 \text { months, have you sought negative feedback about } \\
\text { yourself, from others, or external sources? Some examples of seeking } \\
\text { negative information include: 1) asking your classmate how you did } \\
\text { on a presentation with the hope that he will tell you that you did } \\
\text { poorly on the presentation (e.g., you were stuttering, seemed } \\
\text { unprepared), 2) asking your partner if he/she really loves you with } \\
\text { the hope that your partner will express dislike or hostility towards } \\
\text { you, and 3) asking your friend if you made the correct decision with } \\
\text { the hope that she will inform you that you made the wrong decision. } \\
\text { IF YES: Please explain: }\end{array}$ & $\mathrm{N}$ & $\mathrm{Y}$ \\
\hline
\end{tabular}

IF YES TO ONE OR BOTH: Proceed to the MINI.

IF NO TO BOTH: Unfortunately, you are not eligible to participate in this study. Thank you for your interest and for taking the time to answer those questions.

\section{ADMINISTER THE MINI.}

IF NOT ELIGIBLE: Unfortunately, you are not eligible to participate in this study. Thank you for your interest and for taking the time to answer those questions. Also, your responses indicate that you may be experiencing some difficulties. Would you like me to provide you with some helpful resources?

IF YES: Provide contact information. 


\begin{tabular}{|l|l|}
\hline Centre for Addiction and Mental Health (OHIP-covered) & $416-979-6819$ \\
\hline Clinic on Dupont (Private) & $416-966-1692$ \\
\hline Cognitive and Interpersonal Therapy Centre (Private) & $416-570-5050$ \\
\hline $\begin{array}{l}\text { Cognitive Behavioural Therapy Associates of Toronto } \\
\text { (Private) }\end{array}$ & $416-363-4228$ \\
\hline Sunnybrook CBT Clinic (OHIP-covered) & $416-480-5836$ \\
\hline Humber River Regional Hospital (OHIP-covered) & $416-658-2003$ \\
\hline
\end{tabular}

For further referral options, visit http://martinantony.com/resources/anxiety-referrals/

IF NO: If you change your mind, helpful resources can be found online.

Do you have any concerns about this part of the study?

IF YES: Following is the contact information of the Chair of Research Ethics at Ryerson University: Dr. Lynn Lavellée, 416-979-5000 x 4791

NOTE: Below are additional questions about disorders that are not assessed by the MINI. If not already ineligible, ask the following questions.

\section{(c) Specific Phobia}

1. Have you ever been afraid or nervous when exposed to other specific objects or situations, such as animals (e.g., snakes, bugs, mice, dogs, cats, etc.), seeing blood, getting injections, dentists, heights, storms, water, flying, driving, or enclosed places?

$$
\text { No (go to "BDD") }
$$

Yes (please explain

2. Did it bother you that you felt uncomfortable in this situation?

No

Yes (please explain

3. Did your fear of or with friends and family)? ever interfere with your life (e.g., at work, school, leisure, No

Yes (please explain

Presence of an excessive specific fear: No 


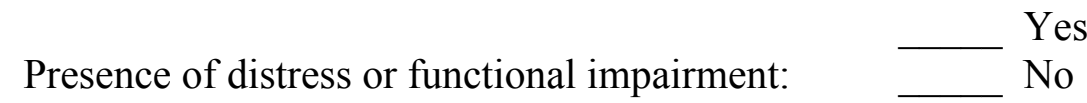

IF SPECIFIC PHOBIA NOT LIKELY: Proceed to BDD.

Yes (rule out of study)

IF SPECIFIC PHOBIA IS PRINCIPAL DIAGNOSIS: Refer to exclusion script above.

(d) Body Dysmorphic Disorder

1. Have you ever been very preoccupied with one or more parts of your physical appearance that you believed was flawed?

No (go to "Hoarding Disorder")

Yes (please explain

2. Did you engage in repetitive behaviours (e.g., checking) or mental acts (e.g., comparing) in response to this preoccupation?

No (go to "Hoarding Disorder")

Yes (please explain

3. Did it bother you that you were preoccupied with these parts of your physical appearance?

No

Yes (please explain

4. Did your preoccupation ever interfere with your life (e.g., at work, school, leisure, or with friends and family)?

No

Yes (please explain

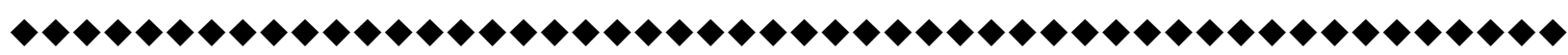

Presence of body dysmorphic concerns: No

Presence of distress or functional impairment:_ _ Y Yes

No

IF BDD NOT LIKELY: Proceed to Hoarding Disorder. Yes (rule out of study)

IF BDD IS PRINCIPAL DIAGNOSIS: Refer to exclusion script above.

\section{(e) Hoarding Disorder}

1. Have you ever had difficulty getting rid of possessions because you felt a need to save them, leading to clutter that compromised the use of living areas?

No (go to "Trichotillomania")

Yes (please explain

2. Did difficulties getting rid of possessions or the clutter bother you?

No

Yes (please explain 
3. Did difficulties getting rid of possessions or the clutter ever interfere with your life (e.g., at work, school, leisure, or with friends and family)?

No

Yes (please explain

Presence of hoarding: No

Presence of distress or functional impairment: $\quad-$ Yes

IF HOARDING NOT LIKELY: Proceed to Trichotillomania.

IF HOARDING IS PRINCIPAL DIAGNOSIS: Refer to exclusion script above.

(f) Trichotillomania

1. Have you ever repeatedly pulled out your hair to the point of causing hair loss? No (go to "Excoriation Disorder")

Yes (please explain

2. Did you repeatedly try to stop or cut down on this behaviour?

No (go to "Excoriation Disorder")

Yes (please explain

3. Did the hair pulling bother you?

No

Yes (please explain

3. Did the hair pulling ever interfere with your life (e.g., at work, school, leisure, or with friends and family)?

No

Yes (please explain )

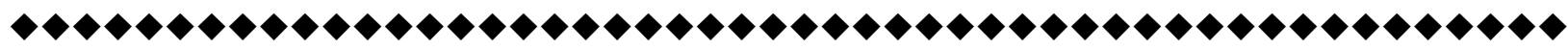

Presence of trichotillomania:

No

Presence of distress or functional impairment: $\quad \longrightarrow$ Nos

Yes (rule out of study)

IF TRICHOTILLOMANIA NOT LIKELY: Proceed to Excoriation Disorder.

IF TRICHOTILLOMANIA IS PRINCIPAL DIAGNOSIS: Refer to exclusion script above.

(g) Excoriation Disorder

1. Have you ever repeatedly picked your skin to the point of causing skin lesions?

No (go to "PTSD")

Yes (please explain 
2. Did you repeatedly try to stop or cut down on this behaviour? No (go to "PTSD")

Yes (please explain

3. Did the skin picking bother you?

No

Yes (please explain

3. Did the skin picking ever interfere with your life (e.g., at work, school, leisure, or with friends and family)?

No

Yes (please explain

Presence of excoriation disorder:

No

Presence of distress or functional impairment: $\quad-$ Yes

IF EXCORIATION NOT LIKELY: Proceed to Wrap-Up. Yes (rule out of study)

IF EXCORIATION IS PRINCIPAL DIAGNOSIS: Refer to exclusion script above.

IF NO SYMPTOMS ENDORSED: Proceed to Wrap-up.

IF SUBSTANCE USE PROBLEMS LIKELY: Refer to exclusion script above.

4) WRAP-UP

IF THE PARTICIPANT IS ELIGIBLE: According to your responses, you are eligible to participate in this study. Now we can set up an appointment for you to come in. The lab is located in the Psychology Research and Training Centre at 105 Bond Street on the first floor.

The room number is SBB114. The phone number is 416-979-5000 x 2184.

Also, your responses indicate that you may be experiencing some difficulties right now. Would you like me to provide you with some helpful resources that you can review before we meet?

IF YES: Provide contact information that is outlined above.

IF NO: If you change your mind, helpful resources can be found online.

\section{Set up an appointment:}

o Date:

o Time:

o Check email 
Appendix B: Feedback Seeking Diary

Name__ Date__time of Recording Feedback Seeking Diary

1. What type of feedback seeking behaviour did you engage in (see definitions on reverse side)?

Positive feedback seeking

Negative feedback seeking

2. What triggered you to seek feedback? Please describe in detail.

3. On which of the following topics/domains did your feedback seeking focus? Check all that apply.

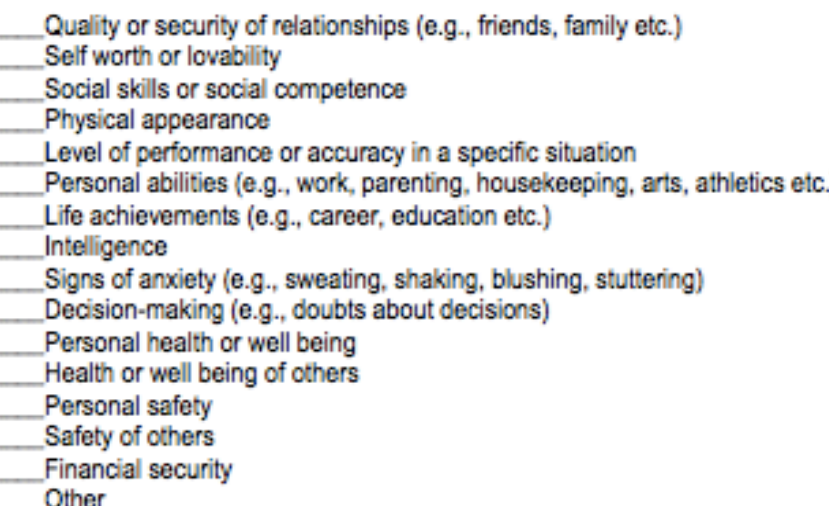

Please describe in detail the topics/domains on which your feedback seeking focused.

4. Who did you seek feedback from?

Romantic partner
Family member; specify
Friend
Acquaintance
$\quad$ Stranger; specify
$\quad$ Oneself
$\quad$ Professionalvauthority figure (e.g., lawyer, doctor, priest, manager etc.); specify
$\quad$ Sources other than people (e.g., websites, books etc.); specify

Sources other than people (e.g., websites, books etc.); specify Other, specify.
Time of Feedback Seeking

5. Please rate each items $5(a)$ and $5(b)$ on the following scale:
Not at all
0
Somewhat
Extremely

3

(a) Just before seeking feedback, I was feeling $(0-6)$ :

1. Fearful

2. Anxious

3. Sad

4. Uncertain

5. Frustrated

6. Disgusted

7. Angry

8. Ashamed

9. Guilty

10. Certain

11. Confident

12. Relaxed

13. Happy

14. Other

$\begin{array}{llllll}0 & 1 & 2 & 3 & 4 & 5 \\ 0 & 1 & 2 & 3 & 4 & 5 \\ 0 & 1 & 2 & 3 & 4 & 5 \\ 0 & 1 & 2 & 3 & 4 & 5 \\ 0 & 1 & 2 & 3 & 4 & 5 \\ 0 & 1 & 2 & 3 & 4 & 5 \\ 0 & 1 & 2 & 3 & 4 & 5 \\ 0 & 1 & 2 & 3 & 4 & 5 \\ 0 & 1 & 2 & 3 & 4 & 5 \\ 0 & 1 & 2 & 3 & 4 & 5 \\ 0 & 1 & 2 & 3 & 4 & 5 \\ 0 & 1 & 2 & 3 & 4 & 5 \\ 0 & 1 & 2 & 3 & 4 & 5 \\ 0 & 1 & 2 & 3 & 4 & 5\end{array}$

(b) Just after receiving feedback, I felt (0-6):

NOTE: If you did not receive any feedback, skip this item, skip \#6, and go to \#7.

$\begin{array}{llllllll}\text { 1. Fearful } & 0 & 1 & 2 & 3 & 4 & 5 & 6 \\ \text { 2. Anxious } & 0 & 1 & 2 & 3 & 4 & 5 & 6 \\ \text { 3. Sad } & 0 & 1 & 2 & 3 & 4 & 5 & 6 \\ \text { 4. Uncertain } & 0 & 1 & 2 & 3 & 4 & 5 & 6 \\ \text { 5. Frustrated } & 0 & 1 & 2 & 3 & 4 & 5 & 6 \\ \text { 6. Disgusted } & 0 & 1 & 2 & 3 & 4 & 5 & 6 \\ \text { 7. Angry } & 0 & 1 & 2 & 3 & 4 & 5 & 6 \\ \text { 8. Ashamed } & 0 & 1 & 2 & 3 & 4 & 5 & 6\end{array}$




$\begin{array}{llllllll}\text { 9. Guilty } & 0 & 1 & 2 & 3 & 4 & 5 & 6 \\ \text { 10.Certain } & 0 & 1 & 2 & 3 & 4 & 5 & 6 \\ \text { 11. Confident } & 0 & 1 & 2 & 3 & 4 & 5 & 6 \\ \text { 12. Relaxed } & 0 & 1 & 2 & 3 & 4 & 5 & 6 \\ \text { 13. Happy } & 0 & 1 & 2 & 3 & 4 & 5 & 6 \\ \text { 14.Other_L } & 0 & 1 & 2 & 3 & 4 & 5 & 6\end{array}$

6. How much do you trust and/or believe the received feedback?

Not at all

Somewhat

0

1

5

7. (a) What kind of feedback were you expecting to receive? Negaive feedback

Positive feedback

Neutral feedback

(b) What kind of feedback were you hoping to receive?

Negative feedback
Positive feedback

Neutral feedback

(c) What kind of feedback did you actually receive? NOTE: Skip this item if you did not receive any feedback.

Negative feedback

Positive feedback

Neutral feedback

8. Please describe in detail why you sought the feedback to begin with (e.g., what motivated you? What were you hoping would happen?)

9. Please describe in detail what caused you to stop seeking feedback.

\section{DESCRIPTIONS OF FEEDBACK SEEKING BEHAVIOURS}

Positive Feedback Seeking involves seeking positive, reassuring information from others (e.g., partner, family), oneself (e.g., reassuring self-talk), or other sources (e.g., websites, books). Seeking positive feedback from others is often in the form of direct verbal requests. When individuals engage in positive feedback seeking they want to receive positive self-relevant information. Some examples include: 1) asking your classmate how you did on a presentation with the hope that he will provide reassurance that you did well, 2) asking your partner if he/she really loves you with the hope that your partner will affirm their love for you, and 3) asking your friend if you made the right decision with the hope that she will reassure you that you did.

Negative Feedback Seeking involves seeking negative information about oneself from others (e.g., partner, family), oneself (e.g., negative self-talk), or other sources (e.g., websites, books). Seeking negative feedback from others is often in the form of direct verbal requests. When individuals engage in this type of feedback seeking they want to receive negative self-relevant information. Some examples include: 1) asking your classmate how you did on a presentation with the hope that he will tell you that you did poorly on the presentation (e.g., you were stuttering, seemed unprepared), 2) asking your partner if he/she really loves you with the hope that your partner will express dislike or hostility towards you, and 3) asking your friend if you made the correct decision with the hope that she will inform you that you made the wrong decision. Although wanting and seeking negative information may seem counterintuitive, there are a number of reasons why individuals engage in this type of behaviour (e.g., to confirm negative self-beliefs, to ensure that interaction partners are aware of their shortcomings etc.).

Neutral Feedback refers to feedback from others (e.g., partner, family), oneself (e.g., neutral self-talk), or other sources (e.g., websites, books) that is neither clearly positive nor clearly negative. 
Appendix C: Positive Feedback Seeking Frequency Questionnaire

\section{Positive Feedback Seeking Frequency Questionnaire (PFSF-Q)}

Instructions: Read the following definition of "Positive Feedback Seeking" before completing this measure.

Positive Feedback Seeking involves seeking positive, reassuring information from others (e.g., partner, family), oneself (e.g., reassuring self-talk), or other sources (e.g., websites, books). Seeking positive feedback from others is often in the form of direct verbal requests. When individuals engage in positive feedback seeking they want to receive positive self-relevant information. Some examples include: 1) asking your classmate how you did on a presentation with the hope that he will provide reassurance that you did well, 2) asking your partner if he/she really loves you with the hope that your partner will affirm their love for you, and 3) asking your friend if you made the right decision with the hope that she will reassure you that you did.

Instructions: Rate each item on a scale from 0 (Never) to 4 (Very Often) based on how often you seek positive feedback about each topic listed below.

\begin{tabular}{|c|c|c|c|c|}
\hline Never & Rarely & Sometimes & Often & Very Often \\
\hline 0 & 1 & 2 & 3 & 4 \\
\hline
\end{tabular}

How often do you seek positive feedback about each of the following topics?

\begin{tabular}{|l|lllll|}
\hline $\begin{array}{l}\text { 1. Quality or security of relationships (e.g., friends, family } \\
\text { etc.) }\end{array}$ & $\begin{array}{l}0 \\
\text { 2. }\end{array}$ & 2 & 3 & 4 \\
\hline 2. Self worth or lovability & 0 & 1 & 2 & 3 & 4 \\
\hline 3. Social skills or social competence & 0 & 1 & 2 & 3 & 4 \\
\hline 4. Physical appearance & 0 & 1 & 2 & 3 & 4 \\
\hline 5. Level of performance or accuracy in a specific situation & 0 & 1 & 2 & 3 & 4 \\
\hline $\begin{array}{l}\text { 6. Personal abilities (e.g., work, parenting, housekeeping, } \\
\text { arts, athletics etc.) }\end{array}$ & 0 & 1 & 2 & 3 & 4 \\
\hline
\end{tabular}




\begin{tabular}{|c|c|c|c|c|c|}
\hline 7. Life achievements (e.g., career, education) & 0 & 1 & 2 & 3 & 4 \\
\hline 8. Intelligence & 0 & 1 & 2 & 3 & 4 \\
\hline $\begin{array}{l}\text { 9. Signs of anxiety (e.g., sweating, shaking, blushing, } \\
\text { stuttering) }\end{array}$ & 0 & 1 & 2 & 3 & 4 \\
\hline 10. Decision-making (e.g., doubts about decisions) & 0 & 1 & 2 & 3 & 4 \\
\hline 11. Personal health or well being & 0 & 1 & 2 & 3 & 4 \\
\hline 12. Health or well being of others & 0 & 1 & 2 & 3 & 4 \\
\hline 13. Personal safety & 0 & 1 & 2 & 3 & 4 \\
\hline 14. Safety of others & 0 & 1 & 2 & 3 & 4 \\
\hline 15. Financial security & 0 & 1 & 2 & 3 & 4 \\
\hline 16. Other & 0 & 1 & 2 & 3 & 4 \\
\hline
\end{tabular}

Instructions: Rate each item on a scale from 0 (Never) to 4 (Very Often) based on how frequently you seek positive feedback from each source listed below.

\begin{tabular}{|c|c|c|c|c|}
\hline Never & Rarely & Sometimes & Often & Very Often \\
\hline 0 & 1 & 2 & 3 & 4 \\
\hline
\end{tabular}

How often do you seek positive feedback from each of the following sources?

$\begin{array}{lllll}0 & 1 & 2 & 3 & 4\end{array}$




\begin{tabular}{|c|c|c|c|c|c|}
\hline 18. Family member; specify & 0 & 1 & 2 & 3 & 4 \\
\hline 19. Friend & 0 & 1 & 2 & 3 & 4 \\
\hline 20. Acquaintance & 0 & 1 & 2 & 3 & 4 \\
\hline 21. Stranger; specify & 0 & 1 & 2 & 3 & 4 \\
\hline 22. Oneself & 0 & 1 & 2 & 3 & 4 \\
\hline $\begin{array}{l}\text { 23. Professional/authority figure (e.g., lawyer, doctor, priest, } \\
\text { manager etc.); specify }\end{array}$ & 0 & 1 & 2 & 3 & 4 \\
\hline $\begin{array}{l}\text { 24. Sources other than people (e.g., websites, books etc.); } \\
\text { specify }\end{array}$ & 0 & 1 & 2 & 3 & 4 \\
\hline 25. Other & 0 & 1 & 2 & 3 & 4 \\
\hline
\end{tabular}


Appendix D: Negative Feedback Seeking Frequency Questionnaire

Negative Feedback Seeking Frequency Questionnaire (NFSF-Q)

Instructions: Read the following definition of "Negative Feedback Seeking" before completing this measure.

Negative Feedback Seeking involves seeking negative information about oneself from others (e.g., partner, family), oneself (e.g., negative self-talk), or other sources (e.g., websites, books). Seeking negative feedback from others is often in the form of direct verbal requests. When individuals engage in this type of feedback seeking they want to receive negative self-relevant information. Some examples include: 1) asking your classmate how you did on a presentation with the hope that he will tell you that you did poorly on the presentation (e.g., you were stuttering, seemed unprepared), 2) asking your partner if he/she really loves you with the hope that your partner will express dislike or hostility towards you, and 3) asking your friend if you made the correct decision with the hope that she will inform you that you made the wrong decision.

Instructions: Rate each item on a scale from 0 (Never) to 4 (Very Often) based on how often you seek negative feedback about each topic listed below.

\begin{tabular}{|c|c|c|c|c|}
\hline Never & Rarely & Sometimes & Often & Very Often \\
\hline 0 & 1 & 2 & 3 & 4 \\
\hline
\end{tabular}

How often do you seek negative feedback about each of the following topics?

\begin{tabular}{|c|c|c|c|c|c|}
\hline $\begin{array}{l}\text { 1. Quality or security of relationships (e.g., family, } \\
\text { friends etc.) }\end{array}$ & 0 & 1 & 2 & 3 & 4 \\
\hline 2. Self worth or lovability & 0 & 1 & 2 & 3 & 4 \\
\hline 3. Social skills or social competence & 0 & 1 & 2 & 3 & 4 \\
\hline 4. Physical appearance & 0 & 1 & 2 & 3 & 4 \\
\hline 5. Level of performance or accuracy in a specific situation & 0 & 1 & 2 & 3 & 4 \\
\hline
\end{tabular}




\begin{tabular}{|c|c|c|c|c|c|}
\hline $\begin{array}{l}\text { 6. Personal abilities (e.g., work, parenting, housekeeping, } \\
\text { arts, athletics etc.) }\end{array}$ & 0 & 1 & 2 & 3 & 4 \\
\hline 7. Life achievements (e.g., career, education etc.) & 0 & 1 & 2 & 3 & 4 \\
\hline 8. Intelligence & 0 & 1 & 2 & 3 & 4 \\
\hline $\begin{array}{l}\text { 9. Signs of anxiety (e.g., sweating, shaking, blushing, } \\
\text { stuttering) }\end{array}$ & 0 & 1 & 2 & 3 & 4 \\
\hline 10. Decision-making (e.g., doubts about decisions) & 0 & 1 & 2 & 3 & 4 \\
\hline 11. Personal health or well being & 0 & 1 & 2 & 3 & 4 \\
\hline 12. Health or well being of others & 0 & 1 & 2 & 3 & 4 \\
\hline 13. Personal safety & 0 & 1 & 2 & 3 & 4 \\
\hline 14. Safety of others & 0 & 1 & 2 & 3 & 4 \\
\hline 15. Financial security & 0 & 1 & 2 & 3 & 4 \\
\hline 16. Other & 0 & 1 & 2 & 3 & 4 \\
\hline
\end{tabular}

Instructions: Rate each item on a scale from 0 (Never) to 4 (Very Often) based on how often you seek negative feedback from each source listed below.

\begin{tabular}{|c|c|c|c|c|}
\hline Never & Rarely & Sometimes & Often & Very Often \\
\hline 0 & 1 & 2 & 3 & 4 \\
\hline
\end{tabular}

How often do you seek negative feedback from each of the following sources? 


\begin{tabular}{|c|c|c|c|c|c|}
\hline 17. Romantic partner & 0 & 1 & 2 & 3 & 4 \\
\hline 18. Family member; specify & 0 & 1 & 2 & 3 & 4 \\
\hline 19. Friend & 0 & 1 & 2 & 3 & 4 \\
\hline 20. Acquaintance & 0 & 1 & 2 & 3 & 4 \\
\hline 21. Stranger; specify & 0 & 1 & 2 & 3 & 4 \\
\hline 22. Oneself & 0 & 1 & 2 & 3 & 4 \\
\hline $\begin{array}{l}\text { 23. Professional/authority figure (e.g., lawyer, doctor, } \\
\text { priest, manager etc.); specify }\end{array}$ & 0 & 1 & 2 & 3 & 4 \\
\hline $\begin{array}{l}\text { 24. Sources other than people (e.g., websites, books } \\
\text { etc.); specify }\end{array}$ & 0 & 1 & 2 & 3 & 4 \\
\hline 25. Other & 0 & 1 & 2 & 3 & 4 \\
\hline
\end{tabular}




\section{References}

American Psychiatric Association (2013). Diagnostic and statistical manual of mental disorders. $5^{\text {th }}$ ed. Arlington, VA: Author.

American Psychiatric Association (1994). Diagnostic and statistical manual of mental disorders. $4^{\text {th }}$ ed. Washington, DC: Author.

Andrews, G., Hobbs, M. J., Borkovec, T. D., Beesdo, K., Craske, M. G., Heimberg, R. G., .. . Stanley, M. A. (2010). Generalized worry disorder: A review of DSM-IV generalized anxiety disorder and options for DSM-V. Depression and Anxiety, 27, 134-147. Doi:http://dx.doi.org/10.1002/da.20658

Antony, M. M., Bieling, P. J., Cox, B. J., Enns, M. W., \& Swinson, R. P. (1998). Psychometric properties of the 42-item and 21-item versions of the Depression Anxiety Stress Scales in clinical groups and a community sample. Psychological Assessment, 10, 176-181. Doi:http://dx.doi.org/10.1037/1040-3590.10.2.176

Antony, M. M., Coons, M. J., McCabe, R. E., Ashbaugh, A., \& Swinson, R. P. (2006). Psychometric properties of the Social Phobia Inventory: Further evaluation. Behaviour Research and Therapy, 44, 1177-1185. Doi:http://dx.doi.org/10.1016/j.brat.2005.08.013

Antony, M. M., Roth, D., Swinson, R., Huta, V., \& Devins, G. M. (1998). Illness intrusiveness in individuals with panic disorder, obsessive-compulsive disorder, or social phobia. Journal of Nervous and Mental Disease, 186, 311-315.

Antony, M. M., \& Rowa, K. (2008). Social anxiety disorder. Göttingen, Germany: Hogrefe.

Antony, M. M., Rowa, K., Liss, A., Swallow, S. R., \& Swinson, R. P. (2005). Social comparison processes in social phobia. Behavior Therapy, 36, 65-75.

Doi:http://dx.doi.org/10.1016/S0005-7894(05)80055-3 
Antony, M. M., \& Swinson, R. P. (2000). Phobic disorders and panic in adults: A guide to assessment and treatment. Washington, DC: American Psychological Association.

Barrera, T. L., \& Norton, P. J. (2009). Quality of life impairment in generalized anxiety disorder, social phobia, and panic disorder. Journal of Anxiety Disorders, 23, 1086-1090.

Doi:http://dx.doi.org/10.1016/j.janxdis.2009.07.01

Beesdo-Baum, K., Jenjahn, E., Höfler, M., Lueken, U., Becker, E. S., \& Hoyer, J. (2012). Avoidance, safety behavior, and reassurance seeking in generalized anxiety disorder. Depression and Anxiety, 29, 948-957. Doi:http://dx.doi.org/10.1002/da.21955

Behar, E., Alcaine, O., Zuellig, A. R., Borkovec, T. D. (2003). Screening for generalized anxiety disorder using the Penn State Worry Questionnaire: A receiver operating characteristic analysis. Journal of Behavior Therapy and Experimental Psychiatry, 34, 25-43.

Birnie, K. A., Sherry, S. B., Doucette, S., Sherry, D. L., Hadjistavropoulos, H. D., \& Stewart, S. H. (2013). The interpersonal model of health anxiety: Testing predicted paths and model specificity. Personality and Individual Differences, 54, 856-861.

Doi:http://dx.doi.org/10.1016/j.paid.2012.12.028

Boelen, P. A., \& Reijntjes, A. (2009). Intolerance of uncertainty and social anxiety. Journal of Anxiety Disorders, 23, 130-135.

Buhr, K., \& Dugas, M. J. (2002). The Intolerance of Uncertainty Scale: Psychometric properties of the English version. Behaviour Research and Therapy, 40, 931-946. Doi:http://dx.doi.org/10.1016/S0005-7967(01)00092-4

Casbon, T. S., Burns, A. B., Bradbury, T. N., \& Joiner, T. E., Jr. (2005). Receipt of negative feedback is related to increased negative feedback seeking among individuals with depressive symptoms. Behaviour Research and Therapy, 43, 485-504. 
Doi:http://dx.doi.org/10.1016/j.brat.2004.03.009

Cassidy, J., Lichtenstein-Phelps, J., Sibrava, N. J., Thomas, C. L., Jr., \& Borkovec, T. D. (2009). Generalized anxiety disorder: Connections with self-reported attachment. Behavior Therapy, 40, 23-38. Doi:http://dx.doi.org/10.1016/j.beth.2007.12.004

Clark, D., M. (1999). Anxiety disorders: Why they persist and how to treat them. Behaviour Research and Therapy, 31, 5-27.

Clark, L. A., \& Watson, D. (1991). Tripartite model of anxiety and depression: Psychometric evidence and taxonomic implications. Journal of Abnormal Psychology, 100, 316-336. Doi:http://dx.doi.org/10.1037/0021-843X.100.3.316

Clark, D. M., \& Wells, A. (1995). A cognitive model of social phobia. In Heimberg, R.G., Liebowitz, M. R., Hope, D. A. \& Schneier, F. R. (eds.) Social phobia: Diagnosis, assessment, and treatment (pp. 69-93). New York, NY: Guilford Press.

Connor, K. M., Davidson, J. R. T., Churchill, L. E., Sherwood, A., Foa, E., \& Weisler, R. H. (2000). Psychometric properties of the Social Phobia Inventory (SPIN): New self-rating scale. British Journal of Psychiatry, 176, 379-386. Retrieved from http://ezproxy.lib.ryerson.ca/login?url=http://search.proquest.com/docview/619455075?a ccountid $=13631$

Cougle, J. R., Fitch, K. E., Fincham, F. D., Riccardi, C. J., Keough, M. E., \& Timpano, K. R. (2012). Excessive reassurance seeking and anxiety pathology: Tests of incremental associations and directionality. Journal of Anxiety Disorders, 26, 117-125. Doi:http://dx.doi.org/10.1016/j.janxdis.2011.10.001

Cox, B. J., Ross, L., Swinson, R. P., Derenfeld, D. M. (1998). A comparison of social phobia outcome measures in cognitive-behavioral group therapy. Behavior Modification, 22, 
285-297.

Coyne, J. C. (1976b). Toward an interactional description of depression. Psychiatry, 39, $28-40$.

Deschênes, S., Dugas, M., Fracalanza, K., \& Koerner, N. (2012). The role of anger in generalized anxiety disorder. Cognitive Behaviour Therapy, 41, 261-271.

doi:http://dx.doi.org/10.1080/16506073.2012.666564

Dugas, M. J., Gagnon, F., Ladouceur, R., \& Freeston, M. H. (1998). Generalized anxiety disorder: A preliminary test of a conceptual model. Behaviour Research and Therapy, 36, 215-226. Retrieved from http://ezproxy.lib.ryerson.ca/login?url=http://search.proquest.com/docview/619334471?a ccountid $=13631$

Dugas, M. J., Hedayati, M., Karavidas, A., Buhr, K., Francis, K., \& Phillips, N. A. (2005). Intolerance of uncertainty and information processing: Evidence of biased recall and interpretations. Cognitive Therapy and Research, 29, 57-70.

Doi:http://dx.doi.org/10.1007/s10608-005-1648-9

Dugas, M. J., \& Robichaud, M. (2007). Cognitive-behavioral treatment for generalized anxiety disorder: from science to practice. New York, NY: Routledge.

Evraire, L. E., \& Dozois, D. J. A. (2011). An integrative model of excessive reassurance seeking and negative feedback seeking in the development and maintenance of depression. Clinical Psychology Review, 31, 1291-1303. Doi:http://dx.doi.org/10.1016/j.cpr.2011.07.014

Erwin, B. A., Heimberg, R. G., Schneier, F. R., \& Liebowitz, M. R. (2003). Anger experience and expression in social anxiety disorder: Pretreatment profile and predictors of attrition 
and response to cognitive-behavioral treatment. Behavior Therapy, 34, 331-350.

Field, A. (2009). Discovery statistics using SPSS, $3^{\text {rd }}$ ed. London, UK: Sage Publications.

Fredrickson, B. L. (2000). Cultivating positive emotions to optimize health and well-being. Prevention and Treatment, 3.

Fresco, D. M., Mennin, D. S., Heimberg, R. G., \& Turk, C. L. (2003). Using the Penn State Worry Questionnaire to identify individuals with generalized anxiety disorder: a receiver operating characteristic analysis. Journal of Behavior Therapy and Experimental Psychiatry, 34, 283-291. doi:http://dx.doi.org/10.1016/j.jbtep.2003.09.001

Gilbert, P. (2001). Evolution and social anxiety: the role of attraction, social competition, and social hierarchies. Psychiatric Clinics of North America, 24, 723-751.

Gratz, K. L., \& Roemer, L. (2004). Multidimensional assessment of emotion regulation and dysregulation: Development, factor structure, and initial validation of the Difficulties in Emotion Regulation Scale. Journal of Psychopathology and Behavioral Assessment, 26, $41-54$.

Grös, D. F., Antony, M. M., Simms, L. J., \& McCabe, R. E. (2007). Psychometric properties of the State-Trait Inventory for Cognitive and Somatic Anxiety (STICSA): Comparison to the State-Trait Anxiety Inventory (STAI). Psychological Assessment, 19, 369-381. doi:http://dx.doi.org/10.1037/1040-3590.19.4.369

Grös, D. F., Simms, L. J., \& Antony, M. M. (2010). Psychometric properties of the State-Trait Inventory for Cognitive and Somatic Anxiety (STICSA) in friendship dyads. Behavior Therapy, 41, 277-284. doi:http://dx.doi.org/10.1016/j.beth.2009.07.001 
Gunthert, K. C., \& Wenze, S. J. (2012). Daily diary methods. In M. R. Mehl \& T. S. Conner (Eds.), Handbook of research methods for studying daily life (pp. 144-159). New York: NY: Guilford Press.

Hackmann, A., Surway, C., \& Clark, D. M. (1998). Seeing yourself through others' eyes: A study of spontaneously occurring images in social phobia. Behavioural and Cognitive Psychotherapy, 26, 3-12.

Heerey, E. A., \& Kring, A. M. (2007). Interpersonal consequences of social anxiety. Journal of Abnormal Psychology, 116, 125-134. doi:http://dx.doi.org/10.1037/0021-843X.116.1.125

Helbig-Lang, S., \& Petermann, F. (2010). Tolerate or eliminate? A systematic review on the effects of safety behavior across anxiety disorders. Clinical Psychology: Science and Practice, 17, 218-233. doi:http://dx.doi.org/10.1111/j.1468-2850.2010.01213.x

Hirsch, C. R., Meynen, T., \& Clark, D. M. (2004). Negative self-imagery in social anxiety contaminates social interactions. Memory, 12, 496-506.

Hofmann, S. G. (2007). Cognitive factors that maintain social anxiety disorder: a comprehensive model and its treatment implications. Cognitive Behaviour Therapy, 4, 193-209.

Joiner, T. E. (2000). Depression's vicious scree: Self-propagating and erosive processes in depression chronicity. Clinical Psychology: Science and Practice, 7, 203-218.

Joiner, T. E., Jr., Alfano, M. S., \& Metalsky, G. I. (1992). When depression breeds contempt: Reassurance seeking, self-esteem, and rejection of depressed college students by their roommates. Journal of Abnormal Psychology, 101, 165-173.

Joiner, T. E., Alfano, M. S., \& Metalsky, G. I. (1993). Caught in the crossfire: Depression, selfconsistency, self-enhancement, and the response of others. Journal of Social and Clinical Psychology, 12, 113-134. 
Joiner, T. E., Jr., Katz, J., \& Lew, A. S. (1997). Self-verification and depression among youth psychiatric inpatients. Journal of Abnormal Psychology, 106, 608-618. doi:http://dx.doi.org/10.1037/0021-843X.106.4.608

Joiner, T. E., \& Metalsky, G. I. (1995). A prospective test of an integrative interpersonal theory of depression: A naturalistic study of college roommates. Journal of Personality and Social Psychology, 69, 778-788. doi:http://dx.doi.org/10.1037/0022-3514.69.4.778

Keller, M. B. (2003). The lifelong course of social anxiety disorder: A clinical perspective. Acta Psychiatrica Scandinavica, 108, 85-94. doi:http://dx.doi.org/10.1034/j.16000447.108.s417.6.x

Kessler, R. C. (2003). The impairments caused by social phobia in the general population: Implications for intervention. Acta Psychiatrica Scandinavica, 108, 19-27. doi:http://dx.doi.org/10.1034/j.1600-0447.108.s417.2.x

Kessler, R. C., Berglund, P., Demler, O., Jin, R., Merikangas, K. R., \& Walters, E. E. (2005). Lifetime prevalence and age-of-onset distributions of DSM-IV disorders in the national comorbidity survey replication. Archives of General Psychiatry, 62, 593-603.

Kessler, R. C., Stang, P., Wittchen, H. -., Stein, M., \& Walters, E. E. (1999). Lifetime comorbidities between social phobia and mood disorders in the US national comorbidity survey. Psychological Medicine, 29, 555-567.

Kobori, O., \& Salkovskis, P. M. (2013). Patterns of reassurance seeking and reassurance-related behaviours in OCD and anxiety disorders. Behavioural and Cognitive Psychotherapy, 41, 1-21. doi:http://dx.doi.org/10.1017/S1352465812000665

Koerner, N., \& Dugas, M. J. (2006). A cognitive model of generalized anxiety disorder: The role of intolerance of uncertainty. In G. C. Davey, \& A. Wells (Eds.). Worry and its 
Psychological Disorders: Theory, Assessment, and Treatment. (pp. 201-216). West Sussex, UK: John Wiley and Sons.

Ladouceur, R., Dugas, M. J., Freeston, M. H., Rh Son, J., Blais, F., Boisvert, J. M., Gagnon, F., \& Thibodeau, N. (1999). Specificity of generalized anxiety disorder symptoms and processes. Behavior Therapy, 30, 191-207.

Ladouceur, R., Gosselin, P., \& Dugas, M. J. (2000). Experimental manipulation of intolerance of uncertainty: A study of a theoretical model of worry. Behaviour Research and Therapy, $38,933-941$.

Leary, M. R. (1983). A brief version of the Fear of Negative Evaluation Scale. Personality and Social Psychology Bulletin, 9, 371-375.

Lovibond, P. F., \& Lovibond, S. H. (1995). The structure of negative emotional states: Comparison of the Depression Anxiety Stress Scales (DASS) with the Beck Depression and Anxiety Inventories. Behaviour Research and Therapy, 33, 335-343.

Marganska, A., Gallagher, M., \& Miranda, R. (2013). Adult attachment, emotion dysregulation, and symptoms of depression and generalized anxiety disorder. American Journal of Orthopsychiatry, 83, 131-141.

Mattick, R. P., \& Clark, J. C. (1998). Development and validation of measures of social phobia scrutiny fear and social interaction anxiety. Behaviour Research and Therapy, 36, 455470.

Meyer, T. J., Miller, M. L., Metzger, R. L. \& Borkovec, T. D. (1990). Development and validation of the Penn State Worry Questionnaire. Behaviour Research and Therapy, 28, 487-495. 
McCabe-Bennett, H. (2014). Naturalistic Observation of Perfectionistic Behaviours. Unpublished Master's thesis, Ryerson University, Toronto, Ontario, Canada.

Moore, M. T., Anderson, N. L., Barnes, J. M., Haigh, E. A. P., \& Fresco, D. M. (2014). Using the GAD-Q-IV to identify generalized anxiety disorder in psychiatric treatment seeking and primary care medical samples. Journal of Anxiety Disorders, 28, 25-30.

Moskowitz, D. S., \& Young, S. N. (2006). Ecological momentary assessment: What it is and why it is a method of the future in clinical psychopharmacology. Journal of Psychiatry and Neuroscience, 31, 13-20.

Muse, K., McManus, F., Leung, C., Meghreblian, B., \& Williams, J. M. G. Hypochondriasis: Fact or fiction? A preliminary examination of the relationship between health anxiety and searching for health information on the Internet. Journal of Anxiety Disorders, 26, 189196.

Newman, M. G., Zuellig, A. R., Kachin, K. E., Constantino, M. J., Przeworski, A., Erickson, T., \& Cashman-McGrath, L. (2002). Preliminary reliability and validity of the Generalized Anxiety Disorder Questionnaire-IV: A revised self-report diagnostic measure of generalized anxiety disorder. Behavior Therapy, 33, 215-233. doi:http://dx.doi.org/10.1016/S0005-7894(02)80026-0

North, R. J., \& Swann, W. B., Jr. (2009). Self-verification $360^{\circ}$ : Illuminating the light and dark sides. Self and Identity, 8, 131-146. doi:http://dx.doi.org/10.1080/15298860802501516

Osman, A., Wong, J. L., Bagge, C. L., Freedenthal, S., Gutierrez, P. M., \& Lozano, G. (2012). The Depression Anxiety Stress Scales-21 (DASS-21): Further examination of dimensions, scale reliability, and correlates. Journal of Clinical Psychology, 68, 13221338. doi:http://dx.doi.org/10.1002/jclp.21908 
Page, A. C., Hooke, G. R., \& Morrison, D. L. (2007). Psychometric properties of the Depression Anxiety Stress Scales (DASS) in depressed clinical samples. British Journal of Clinical Psychology, 46, 283-297. doi:http://dx.doi.org/10.1348/014466506X158996

Parrish, C. L., \& Radomsky, A. S. (2006). An experimental investigation of responsibility and reassurance: relationships with compulsive checking. International Journal of Behavioral and Consultation Therapy, 2, 174-191.

Parrish, C. L., \& Radomsky, A. S. (2010). Why do people seek reassurance and check repeatedly? An investigation of factors involved in compulsive behavior in OCD and depression. Journal of Anxiety Disorders, 24, 211-222. doi:http://dx.doi.org/10.1016/j.janxdis.2009.10.010

Perowne, S., \& Mansell, W. (2002). Social anxiety, self-focused attention, and the discrimination of negative, neutral, and positive audience members by their non-verbal behaviours. Behavioural and Cognitive Psychotherapy, 30, 11-23.

Preacher, K. J., Curran, K. J., \& Bauer, D. J. (2006). Computational tools for probing interactions in multiple linear regression, multilevel modeling, and latent curve analysis. Journal of Educational and Behavioral Statistics, 32, 437-448.

Rector, N. A., Kamkar, K., Cassin, S. E., Ayearst, L. E., \& Laposa, J. M. (2011). Assessing excessive reassurance seeking in the anxiety disorders. Journal of Anxiety Disorders, 25, 911-917. doi:http://dx.doi.org/10.1016/j.janxdis.2011.05.003

Ree, M. J., French, D., MacLeod, C., \& Locke, V. (2008). Distinguishing cognitive and somatic dimensions of state and trait anxiety: Development and validation of the State-Trait Inventory for Cognitive and Somatic Anxiety (STICSA). Behavioural and Cognitive Psychotherapy, 36, 313-332. doi:http://dx.doi.org/10.1017/S1352465808004232 
Robichaud, M., \& Dugas, M. J. (2005). Negative problem orientation (part II): Construct validity and specificity to worry. Behaviour Research and Therapy, 43, 403-412. doi:http://dx.doi.org/10.1016/j.brat.2004.02.008

Robinson, C. M., Klenck, S. C., \& Norton, P. J. (2010). Psychometric properties of the Generalized Anxiety Disorder Questionnaire for DSM-IV among four racial groups. Cognitive Behaviour Therapy, 39, 251-261. doi:http://dx.doi.org/10.1080/16506073.2010.486841

Rodebaugh, T. L., Holaway, R. M., \& Heimberg, R. G. (2008). The factor structure and dimensional scoring of the generalized anxiety disorder questionnaire for DSM-IV. Assessment, 15, 343-350. doi:http://dx.doi.org/10.1177/1073191107312547

Rodebaugh, T. L., Woods, C. M., Thissen, D. M., Heimberg, R. G., Chambless, D. L., \& Rapee, R. M. (2004). More information from fewer questions: The factor structure and item properties of the original and Brief Fear of Negative Evaluation Scale. Psychological Assessment, 16, 169-181. doi:http://dx.doi.org/10.1037/1040-3590.16.2.169

Salkovskis, P. M. (1991). The importance of behaviour in the maintenance of anxiety and panic: A cognitive account. Behavioural Psychotherapy, 19, 6-19.

Salkovskis, P. M., Clark, D. M., \& Gelder, M. G. (1996). Cognition-behaviour links in the persistence of panic. Behaviour Research and Therapy, 34, 453-458.

Salkovskis, P. M., \& Warwick, H. M. (1986). Morbid preoccupations, health anxiety and reassurance: A cognitive-behavioural approach to hypochondriasis. Behaviour Research and Therapy, 24, 597-602. 
Schneier, F. R., Johnson, J., Hornig, C. D., Liebowitz, M. R., \& Weissman, M. M. (1992). Social phobia: Comorbidity and morbidity in an epidemiologic sample. Archives of General Psychiatry, 49, 282-288.

Sexton, K. A., \& Dugas, M. J. (2009). Defining distinct negative beliefs about uncertainty: Validating the factor structure of the Intolerance of Uncertainty Scale. Psychological Assessment, 21, 176-186. doi:http://dx.doi.org/10.1037/a0015827

Sheehan, D. V., Lecrubier, Y., Sheehan, K. H., Amorim, P., Janavs, J., Weiller, E., ... Dunbar, G. C. (1998). The Mini-International Neuropsychiatric Interview (MINI): The development and validation of a structured diagnostic psychiatric interview for DSM-IV and ICD-10. Journal of Clinical Psychiatry, 59, 22-33.

Spielberger, C. (1983). Manual for the State-Trait Anxiety Inventory (Rev. ed.). Menlo Park, CA: Mind Garden.

Spitzer, R. L., Williams, J. B. W., Gibbon, M., \& First, M. B. (1990). Structured Clinical Interview for DSM-III-R, Patient Edition (SCID-P). Washington, DC: American Psychiatric Press.

Starr, L. R., \& Davila, J. (2008). Excessive reassurance seeking, depression, and interpersonal rejection: A meta-analytic review. Journal of Abnormal Psychology, 117, 762-775. doi:http://dx.doi.org/10.1037/a0013866

Stein, M. B., \& Kean, Y. M. (2000). Disability and quality of life in social phobia: Epidemiologic findings. American Journal of Psychiatry, 157, 1606-1613.

Swann, W., B. (1993). Self verification: Bringing social reality into harmony with the self. In J. Suls, \& A. G Greenwald (Eds.). Social psychological perspectives on the self, Vol. 2 (pp. 33-66). Hillsdale, NJ: Erlbaum. 
Swann, W. B., Griffin, J. J., Predmore, S. C., \& Gaines, B. (1987). The cognitive-affective crossfire: When self-consistency confronts self-enhancement. Journal of Personality and Social Psychology, 52, 881-889. doi:http://dx.doi.org/10.1037/0022-3514.52.5.881

Swann, W. B., \& Read, S. J. (1981). Acquiring self-knowledge: The search for feedback that fits. Journal of Personality and Social Psychology, 41, 1119-1128. doi:http://dx.doi.org/10.1037/0022-3514.41.6.1119Swann, W.

Swann, W. B., \& Read, S. J. (1981). Self-verification processes: How we sustain our selfconceptions. Journal of Experimental Social Psychology, 17, 351-372. Retrieved from http://ezproxy.lib.ryerson.ca/login?url=http://search.proquest.com/docview/616608095?a countid $=13631$

Swann, W. B., Jr., Ronde, D. L., \& Hixon, J. G. (1994). Authenticity and positivity strivings in marriage and courtship. Journal of Personality and Social Psychology, 66, 857-869. doi:http://dx.doi.org/10.1037/0022-3514.66.5.857

Swann, W. B., Stein-Seroussi, A., \& Giesler, R. B. (1992). Why people self-verify. Journal of Personality and Social Psychology, 62, 392-401. doi:http://dx.doi.org/10.1037/00223514.62.3.392

Swann, W. B., Wenzlaff, R. M., Krull, D. S., \& Pelham, B. W. (1992). Allure of negative feedback: Self-verification strivings among depressed persons. Journal of Abnormal Psychology, 101, 293-306. doi:http://dx.doi.org/10.1037/0021-843X.101.2.293

Schwarz, N. (2012). Why researchers should think "real time": A cognitive rationale. In M. R. Mehl \& T. S. Conner (Eds.), Handbook of research methods for studying daily life (pp. 22-42). New York: NY: Guilford Press. 
Tallis, F., \& Eysenck, M. W. (1994). Worry: Mechanisms and modulating influences. Behavioural and Cognitive Psychotherapy, 22, 37-56.

Townsend, M. H., Weissbaker, K. A., Barbee, J. G., Pena, J. M., Snider, L. M., Tynes, L. L., ...Winstead, D. K. (1999). Compulsive behavior in generalized anxiety disorder and obsessive-compulsive disorder. Journal of Nervous and Mental Disease, 187, 697-699.

Turner, S. M., Johnson, M. R., Beidel, D. C., Heiser, N. A., \& Lydiard, R. B. (2003). The Social Thoughts and Beliefs Scale: A new inventory for assessing cognitions in social phobia. Psychological Assessment, 15, 384-391. doi:http://dx.doi.org/10.1037/10403590.15.3.384

Valentiner, D. P., Skowronski, J. J., McGrath, P. B., Smith, S. A., \& Renner, K. A. (2011). Selfverification and social anxiety: Preference for negative social feedback and low social self-esteem. Behavioural and Cognitive Psychotherapy, 39, 601-617. doi:http://dx.doi.org/10.1017/S1352465811000300

Wallace, S. T., \& Alden, L. E. (1995). Social anxiety and standard setting following social success and failure. Cognitive Therapy and Research, 19, 613-631.

Weeks, J. W. (2015). Replication and extension of a hierarchical model of social anxiety and depression: Fear of positive evaluation as a key factor in social anxiety. Cognitive Behaviour Therapy, 44, 103-116. doi:http://dx.doi.org/10.1080/16506073.2014.990050

Weeks, J. W., Heimberg, R. G., Fresco, D. M., Hart, T. A., Turk, C. L., Schneier, F. R., \& Liebowitz, M. R. (2005). Empirical validation and psychometric evaluation of the brief fear of negative evaluation scale in patients with social anxiety disorder. Psychological Assessment, 17, 179-190. doi:http://dx.doi.org/10.1037/1040-3590.17.2.179 
Weeks, J. W., Heimberg, R. G., \& Rodebaugh, T. L. (2008). The Fear of Positive Evaluation Scale: Assessing a proposed cognitive component of social anxiety. Journal of Anxiety Disorders, 22, 44-55. doi:http://dx.doi.org/10.1016/j.janxdis.2007.08.002

Weeks, J. W., \& Howell, A. N. (2012). The bivalent fear of evaluation model of social anxiety: Further integrating findings on fears of positive and negative evaluation. Cognitive Behaviour Therapy, 41, 83-95. doi:http://dx.doi.org/10.1080/16506073.2012.661452

Wells, A., Clark, D. M., Salkovskis, P., Ludgate, J., Hackmann, A., \& Gelder, M. (1995). Social phobia: The role of in-situation safety behaviors in maintaining anxiety and negative beliefs. Behavior Therapy, 26, 153-161.

Wheeler, L., \& Miyake, K. (1992). Social comparison in everyday life. Journal of Personality and Social Psychology, 62, 760-773. doi:http://dx.doi.org/10.1037/0022-3514.62.5.760

Woody, S., \& Rachman, S. (1994). Generalized anxiety disorder (GAD) as an unsuccessful search for safety. Clinical Psychology Review, 14, 743-753.

World Health Organization (1990). Composite International Diagnostic Interview (CIDI). Version 1.0. Geneva, Switzerland: Author.

World Health Organization (1992). International Classification of Diseases, Tenth Revision. Geneva, Switzerland: Author. 